\title{
CP-odd invariants for multi-Higgs models: applications with discrete symmetry
}

\author{
Ivo de Medeiros Varzielas ${ }^{a *}$, Stephen F. King ${ }^{a \dagger}$, \\ Christoph Luhn ${ }^{b \ddagger}, \quad$ Thomas Neder ${ }^{a}$ \\ ${ }^{a}$ School of Physics and Astronomy, University of Southampton, \\ SO17 1BJ Southampton, United Kingdom \\ ${ }^{b}$ Theoretische Physik 1, Naturwissenschaftlich-Technische Fakultät, Universität Siegen, \\ Walter-Flex-Straße 3, 57068 Siegen, Germany
}

\begin{abstract}
$\mathrm{CP}$-odd invariants provide a basis independent way of studying the $\mathrm{CP}$ properties of Lagrangians. We propose powerful methods for constructing basis invariants and determining whether they are $\mathrm{CP}$-odd or CP-even, then systematically construct all of the simplest $\mathrm{CP}$-odd invariants up to a given order, finding many new ones. The CP-odd invariants are valid for general potentials when expressed in a standard form. We then apply our results to scalar potentials involving three (or six) Higgs fields which form irreducible triplets under a discrete symmetry, including invariants for both explicit as well as spontaneous $\mathrm{CP}$ violation. The considered cases include one triplet of Standard Model (SM) gauge singlet scalars, one triplet of SM Higgs doublets, two triplets of SM singlets, and two triplets of SM Higgs doublets. For each case we study the potential symmetric under one of the simplest discrete symmetries with irreducible triplet representations, namely $A_{4}, S_{4}, \Delta(27)$ or $\Delta(54)$, as well as the infinite classes of discrete symmetries $\Delta\left(3 n^{2}\right)$ or $\Delta\left(6 n^{2}\right)$.
\end{abstract}

\footnotetext{
*E-mail: ivo.de@soton.ac.uk

$\dagger$ E-mail: king@soton.ac.uk

$\ddagger$ E-mail: christoph.luhn@uni-siegen.de

$\S$ E-mail: t.neder@soton.ac.uk
} 


\section{Contents}

1 Introduction 1

2 CP-odd invariants for scalar potentials 3

2.1 General formalism . . . . . . . . . . . . . . . . . . 3

2.2 CP-odd invariants for explicit $\mathrm{CP}$ violation . . . . . . . . . . 7

2.3 Diagrams for invariants . . . . . . . . . . . . . . . . . . . . 10

2.4 CP-odd invariants only built from $Z$ tensors . . . . . . . . . . . . . . . . 11

2.5 CP-odd invariants built from $Y$ and $Z$ tensors . . . . . . . . . . . . . . 13

3 Two Higgs doublet model potential $\quad 14$

$4 A_{4}=\Delta(12)$ invariant potentials $\quad 16$

4.1 One flavour triplet . . . . . . . . . . . . . . . . . . . . . . 16

4.2 One flavour triplet of Higgs doublets . . . . . . . . . . . . . . . . . . 17

4.3 Two flavour triplets . . . . . . . . . . . . . . . . . . . . . 18

4.4 Two flavour triplets of Higgs doublets . . . . . . . . . . . . . . . . . 19

$4.5 S_{4}$ invariant potentials . . . . . . . . . . . . . . . . 21

$5 \Delta(27)$ invariant potentials $\quad 22$

5.1 One flavour triplet . . . . . . . . . . . . . . . . . . . 22

5.2 One flavour triplet of Higgs doublets . . . . . . . . . . . . . . . 24

5.3 Two flavour triplets . . . . . . . . . . . . . . . . . . . . 25

5.4 Two flavour triplets of Higgs doublets . . . . . . . . . . . . . . . 25

$5.5 \Delta(54)$ invariant potentials . . . . . . . . . . . . . . . . 26

$6 \Delta\left(3 n^{2}\right)$ invariant potentials with $n>3 \quad 27$

6.1 One flavour triplet . . . . . . . . . . . . . . . . . . . . . . 27

6.2 One flavour triplet of Higgs doublets . . . . . . . . . . . . . 27

6.3 Two flavour triplets . . . . . . . . . . . . . . . . . . . . . . 28

6.4 Two flavour triplets of Higgs doublets . . . . . . . . . . . . . . . . . . . 29]

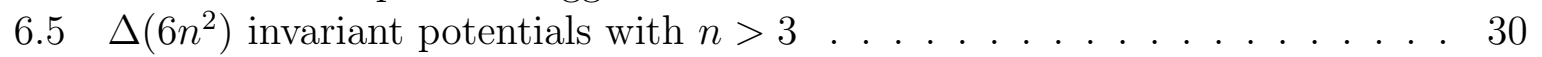

7 Summary of CPIs for explicit CP violation $\quad 30$

8 CP-odd invariants for spontaneous CP violation 31

8.1 Minimisation condition in terms of diagrams . . . . . . . . . . . . 33

8.2 Example applications . . . . . . . . . . . . . . . . . . . . . . . . . . . . . . . . . .

8.2 .1 One triplet of $A_{4} \ldots \ldots \ldots \ldots \ldots$

8.2 .2 One triplet of $\Delta(27) \ldots \ldots \ldots \ldots \ldots$

9 Conclusions

A Symmetries of invariants 
B List of invariants

B.1 Contraction matrices of $n_{Z}=5$ invariants . . . . . . . . . . . . 43

B.2 Contraction matrices of $n_{Z}=6$ invariants without $Z$-self-loops . . . . . . . 43

B.3 $n_{Z}=6$ invariants with self-loops . . . . . . . . . . . . . . 45

B.4 Invariants with $n_{Y} \neq 0$ not listed in the main text . . . . . . . . . . . 46

B.5 Lists of spontaneous CP-odd invariants . . . . . . . . . . . . . . . 48

B.6 Larger CP-odd invariants . . . . . . . . . . . . . . . . . . . . . . 49

C $\Delta\left(6 n^{2}\right)$ potentials as particular cases of $\Delta\left(3 n^{2}\right)$ potentials 50

\section{Introduction}

CP symmetry, which is just the combination of particle-antiparticle exchange and space inversion, is presently known to be violated only by the weak interactions involving quarks in the Standard Model (SM) [1]. The origin of the observed SM quark CP violation (CPV) is a natural consequence of three generations of quarks whose mixing is described by a complex CKM matrix. Although the CKM matrix can be parameterised in different ways, it was realised that the amount of CPV in physical processes always depends on a particular weak basis invariant which can be expressed in terms of the quark mass matrices [2]. In the SM the electroweak symmetry $S U(2)_{L} \times U(1)_{Y}$ is broken to the electromagnetic gauge group $U(1)_{Q}$ by a single Higgs doublet, resulting in a single physical Higgs boson which has been observed with a mass near $125 \mathrm{GeV}[3,4]$. Although $\mathrm{CP}$ is automatically conserved by the Higgs potential of the SM, with more than one Higgs doublet it is possible that the Higgs potential violates $\mathrm{CP}$, providing a new source of CPV [5]. This is welcome since Sakharov discovered that CPV is a necessary condition for baryon asymmetry generation $[6]$ and CPV arising from the quark sector of the SM is insufficient [7].

It is also possible, indeed likely, that $\mathrm{CP}$ could be violated in the lepton sector, as is hinted at by global fits [8,9], and such a source of CPV could also contribute to the baryon asymmetry via leptogenesis [10]. In this case one would like to construct models that explain the structure of the lepton mass matrices, through which CPV enters the processes for creating the baryon asymmetry. Typical examples of such models that use discrete symmetries to constrain the structure of mass matrices need several multiplets of scalar fields that also transform under the same symmetry (for reviews, cf. [11 16]). Such models provide a motivation to study multiple SM Higgs singlets (sometimes called "flavons" in this context) as well as electroweak doublets. In the context of flavour models it is natural to consider Higgs doublets or singlets which play the role of "flavons" and form irreducible triplets under some spontaneously broken discrete family symmetry. Motivated by the above considerations, we shall also study CPV Higgs potentials of this type.

As already mentioned in the context of the CKM matrix, the study of CP is a subtle topic because of the basis dependent nature of the phases which control CPV. Similar considerations also apply to the phases which appear in the parameters of the potentials of multiple scalars. 
An important tool to assist in determining whether $\mathrm{CP}$ is violated or not are basis independent CP-odd invariants (CPIs), whose usefulness has been shown in the SM in addressing CP violation arising from the CKM matrix, sourced from the Yukawa couplings. The first example of the use of such invariants was the Jarlskog invariant [2], which was reformulated in [17] in a form which is generally valid for an arbitrary number of generations. Generalising the invariant approach [17] and applying it to fermion sectors of theories with Majorana neutrinos [18] or with discrete symmetries [19,20] leads to other relevant CPIs.

In extensions of the Higgs sector of the SM, the $\mathrm{CP}$ violation arising from the parameters of the scalar potential can be studied in a similar basis invariant way as for the quark sector. For example, in the general two Higgs Doublet Model (HDM) 5] (see 21 for a recent analysis) a CPI was identified in 22]. More generally, applying the invariant approach to scalar potentials has revealed relevant CPIs [23 25], including for the 2HDM [26, 27]. Building on these results, the goal of this work is to consider yet more general Higgs potentials and adopt the powerful method of so-called contraction matrices in order to identify and construct new non-trivial CPIs, which we subsequently apply to potentials involving three or six Higgs fields (which can be either electroweak doublets or singlets) which form irreducible triplets under a discrete symmetry.

We begin by reviewing the systematic approach to CPIs for arbitrary scalar potentials, focusing on renormalisable potentials with quadratic and quartic couplings. The reader may be primarily interested in cases where the Higgs fields are electroweak $S U(2)_{L}$ doublets, but the formalism can also be applied to more general scalar potentials including cases where the Higgs fields are SM singlets. We develop powerful tools where basis invariants 25 27 can be represented pictorially by diagrams (introduced for the 2HDM in [26]) and introduce matrices later designated as contraction matrices, that identify how the parameters in the potential are combined to form a basis invariant. The diagrams and matrices are extremely helpful in distinguishing CPIs from basis invariants that are CP-even, as well as cataloguing each CPI uniquely in association with an element of a group of permutations. We emphasise that CPIs as defined via such matrices are valid for any potential, and then take specific expressions when specialising to a potential (often vanishing for cases where the potential is very symmetric, even if the potential features explicit CP violation as shown by other non-vanishing CPIs).

Having translated the well-known technique for constructing CPIs to diagrams and contraction matrices, we apply this formalism to some physically interesting cases. We begin with the familiar example of the general 2HDM. We then move on to examples of potentials which involve three or six Higgs fields which fall into irreducible triplet representations of discrete symmetries belonging to the $\Delta\left(3 n^{2}\right)$ and $\Delta\left(6 n^{2}\right)$ series studied extensively in the context of flavour and CP models in [28 51]. We consider specific cases of the 3HDM [52 68] and of the 6HDM 69 75] where the three or six Higgses are related by the discrete symmetry as one or two (flavour) triplets. Although many of these cases have already been studied in the literature, our systematic formalism yields many new results. For example, although $\Delta(27)$ with a single triplet of Higgs doublets has been extensively studied in the literature [52, 55, 57, 60, 61, 66], using the invariant approach and the CPIs we are able to identify several new results of interest.

Using the invariant approach, the considered cases include one triplet of SM gauge 
singlets, one triplet of SM Higgs doublets, two triplets of SM singlets, and two triplets of SM Higgs doublets, where for each case we study the potential symmetric under one of the simplest discrete symmetries with irreducible triplet representations, namely $A_{4}, S_{4}$, $\Delta(27)$ or $\Delta(54)$, as well as the infinite classes of discrete symmetries $\Delta\left(3 n^{2}\right)$ or $\Delta\left(6 n^{2}\right)$. In each case, we show which potentials are in general CP conserving (all the CPIs vanish, and we provide a CP symmetry that leaves the potential invariant) or in general CP violating (in which case it is sufficient to show a single non-vanishing $\mathrm{CPI}$ ). For the CP violating potentials we further demonstrate the effects of imposing specific CP symmetries, that in constraining the parameters of the potential in one way or the other, lead all CPIs to vanish. Furthermore, we extend our formalism by including also Vacuum Expectation Values (VEVs), obtaining Spontaneous CPIs (SCPIs) that are non-vanishing if CP is spontaneously violated (as considered earlier in [23,24]). We illustrate one of these SCPIs by applying it to the better studied $\Delta(27)$ potential, exploring different $\mathrm{CP}$ symmetries and VEVs that either conserve or spontaneously violate the imposed CP symmetry.

Since this subject has been studied extensively, it is relevant to clarify what is new in this paper:

- We improve on the diagram-based approach of [26].

- With respect to the existing literature [23 27], we build CPIs up to orders in the bi-linear and quartic scalar couplings which are higher than those of previous work.

- To the best of our knowledge, we discuss for the first time the use of CPIs in the context of discrete symmetries and give extensive examples.

- We introduce a very efficient new method for identifying higher order CPIs based on contraction matrices (in Appendix A).

The layout of the paper is as follows. In Section 2 we cover our general formalism. In Section 3 we revisit the $2 \mathrm{HDM}$ potential showing how our formalism applies. In Section 4, 5 and 6 we apply our techniques respectively to $\Delta\left(3 n^{2}\right)$ and $\Delta\left(6 n^{2}\right)$ groups with $n=2\left(A_{4}, S_{4}\right), n=3(\Delta(27), \Delta(54))$ and $n>3$. A summary of the results obtained for the potentials invariant under discrete symmetries is contained in Section 7 (including Table 1). Section 8 is dedicated to SCPIs. We conclude in Section 9, Further material is included in Appendix A discussing symmetries of basis invariants (including CPIs), Appendix B features a complete list of the CPIs and SCPIs we found and used throughout the paper, and Appendix C discusses how to obtain results for $\Delta\left(6 n^{2}\right)$ from the results of the $\Delta\left(3 n^{2}\right)$ potentials.

\section{CP-odd invariants for scalar potentials}

\subsection{General formalism}

One important aim of this paper is to explore the CP properties of the Higgs sector of models with several copies of SM Higgs doublets. Often, scalar potentials can be confusingly complex and it can be unclear which parameters can contribute to CP violation. 
This situation is made even more difficult by the possibility of choosing different bases which modify the explicit form of the potential but should not change the physics described by it. Both of these difficulties can be overcome by CPIs, in this case CP-odd (Higgs-) basis invariants, that, when non-zero, indicate $\mathrm{CP}$ violation. A similar CPI for the Yukawa sector of the SM is the well-known Jarlskog invariant [2].

Before defining and discussing CPIs of the scalar sector in detail, we first show how to write any possible Higgs potential in a standard form which is suitable to construct general basis invariants. This procedure has the advantage that basis invariants only have to be derived once in the standard form; their explicit form for any particular Higgs potential follows almost trivially by translating the latter into the standard parametrisation. Furthermore, invariants that are CP-odd (CPIs) for the standard form of potentials are so by construction and, if non-zero, indicate $\mathrm{CP}$ violation for all possible example potentials.

The relation between non-zero CPIs and CP violation can be formulated more precisely as follows. If a potential conserves CP, then all CPIs vanish automatically. Reversely, if one or several CPIs are non-zero, the potential violates CP. This statement holds for both explicit and spontaneous CP violation, and the corresponding CPIs are introduced in Sections 2.2 and 8 . Note that CPIs only guarantee $\mathrm{CP}$ conservation if all of them vanish. This is equivalent to demanding a finite set of CPIs, the so-called basis out of which all other CPIs can be produced, to vanish. Such a basis of CPIs is known for the 2HDM [27], but, so far, not for any other more complicated scalar potentials.

In the following, we first introduce the standard form for scalar potentials as discussed in 25, 26. Having established out notation, we analyse the effects of symmetry transformations, general basis transformations, complex conjugation and $\mathrm{CP}$ transformations on the variables and parameters of the standard form. Adopting the procedure and notation of [25, 26], any even potential of $N$ scalar fields $\varphi_{i}$ can, with $\phi=\left(\varphi_{1}, \ldots, \varphi_{N}\right)$ and $\phi^{*}=\left(\varphi_{1}^{*}, \ldots, \varphi_{N}^{*}\right)$, be written as

$$
V_{\text {scalar }}=\phi^{* a} Y_{a}^{b} \phi_{b}+\phi^{* a} \phi^{* c} Z_{a c}^{b d} \phi_{b} \phi_{d}
$$

where the notation is such that lower indices on $Y$ and $Z$ are always contracted with $\phi^{*}$ and upper indices with $\phi . Y$ and $Z$ are tensors that contain all possible couplings and are subject to possible symmetries acting on $\phi$, as will be explained below ${ }^{1}$

Any potential of several Higgs doublets can be brought into this standard form by $\phi$ not containing doublets as such, but instead directly containing the components of the doublets: for $n$ Higgs doublets $H_{i \alpha}=\left(h_{i, 1}, h_{i, 2}\right)$, where $\alpha=1,2$ denotes the $S U(2)_{L}$ index and $i$ goes from 1 to $n$,

$$
\phi=\left(\varphi_{1}, \varphi_{2}, \ldots, \varphi_{2 n-1}, \varphi_{2 n}\right)=\left(h_{1,1}, h_{1,2}, \ldots, h_{n, 1}, h_{n, 2}\right)
$$

and the invariance of the potential under $S U(2)_{L} \times U(1)_{Y}$ will be directly reflected in the structure of $Y$ and $Z$ in a component-wise way. This convention, which differs from the notation of [25, 26], will be very useful later on ${ }^{2}$

\footnotetext{
${ }^{1}$ One could also add a term such as e.g. $T_{c}^{a b} \phi_{a} \phi_{b} \phi^{* c}+$ h.c. to the potential to account for trilinear couplings and the discussion could be extended in this way.

${ }^{2}$ In 26, for example, the $S U(2)_{L}$ indices are summed over outside of $Z$. Our definition of $Z$ ten-
} 
More explicitly, if the theory is invariant under symmetry transformations of a group $G$ such that $\phi$ transforms in some (maybe reducible) representation $\rho(g)$ of that group, where $\rho(g)$ is the matrix that corresponds to the group element $g \in G$,

$$
\begin{aligned}
\phi_{a} & \mapsto[\rho(g)]_{a}^{a^{\prime}} \phi_{a^{\prime}}, \\
\phi^{* a} & \mapsto \phi^{* a^{\prime}}\left[\rho^{\dagger}(g)\right]_{a^{\prime}}^{a},
\end{aligned}
$$

then the invariance of the potential imposes the following constraints on the coupling tensors:

$$
\begin{gathered}
Y_{a}^{b}=\rho_{a}^{a^{\prime}} Y_{a^{\prime}}^{b^{\prime}} \rho_{b^{\prime}}^{\dagger^{\prime}}, \\
Z_{a c}^{b d}=\rho_{a}^{a^{\prime}} \rho_{c}^{c^{\prime}} Z_{a^{\prime} c^{\prime}}^{b^{\prime} d^{\prime}} \rho_{b^{\prime}}^{\dagger^{\dagger}} \rho_{d^{\prime}}^{\dagger^{d}},
\end{gathered}
$$

where we have written $\rho_{a}^{a^{\prime}}=[\rho(g)]_{a}^{a^{\prime}}$ and so on. In addition to that, the quartic coupling tensor $Z_{a c}^{b d}$ is by construction invariant under exchanging $a \leftrightarrow c$ as well as $b \leftrightarrow d$. The reason for this is that $\phi_{b}$ and $\phi_{d}$ commute so that the indices $b$ and $d$ can be renamed into each other to restore the original ordering of the $\phi$ 's, and equivalently for $\phi^{*}$ with $a$ and $c$.

While the theory is invariant under symmetry transformations, one also has the possibility of applying basis transformations under which the Lagrangian is not invariant. Of course, such a basis transformation should not change physics. A simple example is the transformation that diagonalises the bilinear mass terms $\phi^{* a} Y_{a}^{b} \phi_{b}$. As $Z$ is generally only invariant under a smaller group than that of all basis changes, diagonalising $Y$ would change $Z 3^{3}$ Adopting our notation for the standard form of Higgs potentials, a unitary basis transformation in the space of the $N$ dimensional vector $\phi$, i.e. with $V \in U(N)$ a unitary $N \times N$ matrix, maps

$$
\begin{aligned}
\phi_{a} & \mapsto V_{a}^{a^{\prime}} \phi_{a^{\prime}}, \\
\phi^{* a} & \mapsto \phi^{* a^{\prime}} V_{a^{\prime}}^{\dagger a} .
\end{aligned}
$$

With this definition, the kinetic terms remain unchanged while $Y$ and $Z$ transform to

$$
\begin{aligned}
Y_{a}^{b} & \mapsto V_{a}^{a^{\prime}} Y_{a^{\prime}}^{b^{\prime}} V_{b^{\prime}}^{\dagger^{\prime}}, \\
Z_{a c}^{b d} & \mapsto V_{a}^{a^{\prime}} V_{c}^{c^{\prime}} Z_{a^{\prime} c^{\prime}}^{b^{\prime} d^{\prime}} V_{b^{\prime}}^{\dagger^{\prime}} V_{d^{\prime}}^{\dagger d} .
\end{aligned}
$$

Complex conjugation is an essential part of $\mathrm{CP}$ transformations and in the notation used here, changes the vertical position of the index of a field so that

$$
\begin{aligned}
\phi_{a} & \mapsto\left(\phi_{a}\right)^{*} \equiv \phi^{* a}, \\
\phi^{* a} & \mapsto\left(\phi^{* a}\right)^{*} \equiv \phi_{a} .
\end{aligned}
$$

sors can be related to 26 by explicitly highlighting the $S U(2)_{L}$ subindices, $\{1,2, \ldots, 2 n-1,2 n\}=$ $\{(1,1),(1,2), \ldots,(n, 1),(n, 2)\}$. With this, the $Z$ tensors in our paper become $Z_{c d}^{a b}=Z_{(\tilde{c}, \gamma),(\tilde{d}, \delta)}^{(\tilde{a}, \alpha)}=$ $\tilde{Z}_{\tilde{c} \tilde{d}}^{\tilde{a} \tilde{b}} \delta_{\gamma}^{\alpha} \delta_{\delta}^{\beta}$, where $\tilde{Z}$ denotes the coupling tensors of 26 .

${ }^{3}$ Except in the case where the components of $Y$ conspire in such a way that the required basis transformation coincides with a symmetry transformation. Furthermore, a general basis transformation changes the form of the potential, while only transformations in the automorphism group $\operatorname{Aut}(G)$ leave the potential form-invariant. 
Complex conjugating the $Y$ term of the potential then results in

$$
\phi^{* a} Y_{a}^{b} \phi_{b} \mapsto \phi_{a}\left(Y_{a}^{b}\right)^{*} \phi^{* b}=\phi^{* b}\left(Y_{a}^{b}\right)^{*} \phi_{a}=\phi^{* a}\left(Y_{b}^{a}\right)^{*} \phi_{b} .
$$

Comparing this to the original term in the potential and demanding $V_{\text {scalar }}^{*}=V_{\text {scalar }}$ shows that

$$
\left(Y_{b}^{a}\right)^{*}=Y_{a}^{b}
$$

A similar result is obtained for the quartic coupling, i.e.

$$
\left(Z_{b d}^{a c}\right)^{*}=Z_{a c}^{b d}
$$

Note that because both indices of a contracted pair interchange position under complex conjugation, no situation can arise where one would need to sum over two upper or two lower indices. However, expressions as e.g. Eqs. 2.14 and 2.15 where indices appear with exchanged vertical positions without being summed over need to be understood as conditions on the components of the tensors and not the tensors themselves.

Finally, all components are in place to define a (general) CP transformation ${ }^{4}$ with a unitary matrix $U$ on the fields as

$$
\begin{aligned}
\phi_{a} & \mapsto \phi^{* a^{\prime}} U_{a^{\prime}}^{a}, \\
\phi^{* a} & \mapsto U_{a}^{\dagger a^{\prime}} \phi_{a^{\prime}}
\end{aligned}
$$

Again, this leaves the kinetic terms invariant, while applying the CP transformation to the fields in the potential results for the $Y$ term in

$$
\begin{aligned}
\phi^{* a} Y_{a}^{b} \phi_{b} \mapsto U_{a}^{\dagger}{ }_{a}^{a^{\prime}} \phi_{a^{\prime}} Y_{a}^{b} \phi^{* b^{\prime}} U_{b^{\prime}}^{b} & =U_{a}^{\dagger a^{\prime}} \phi_{a^{\prime}}\left(Y_{b}^{a}\right)^{*} \phi^{* b^{\prime}} U_{b^{\prime}}^{b} \\
& =\phi^{* b^{\prime}} U_{b^{\prime}}^{b}\left(Y_{b}^{a}\right)^{*} U_{a}^{\dagger a^{a^{\prime}}} \phi_{a^{\prime}} \\
& =\phi^{* a} U_{a}^{a^{\prime}}\left(Y_{a^{\prime}}^{b^{\prime}}\right)^{*} U_{b^{\prime}}^{\dagger} \phi_{b} .
\end{aligned}
$$

Comparing this to the original term in the potential shows that a CP transformation acting on the fields can be equally understood as the following change of $Y$ (likewise for $Z$ ),

$$
\begin{aligned}
Y_{a}^{b} & \mapsto U_{a}^{a^{\prime}}\left(Y_{a^{\prime}}^{b^{\prime}}\right)^{*} U_{b^{\prime}}^{\dagger^{\prime}} \\
Z_{a c}^{b d} & \mapsto U_{a}^{a^{\prime}} U_{c}^{c^{\prime}}\left(Z_{a^{\prime} c^{\prime}}^{b^{\prime} d^{\prime}}\right)^{*} U_{b^{\prime}}^{\dagger^{b}} U^{\dagger^{d}}{ }_{d^{\prime}}
\end{aligned}
$$

The condition for CP invariance of the standard form of the potential $V_{\text {scalar }}$ in Eq. (2.1), and thus any example potential that can be brought into this standard form, can then be phrased as follows: $C P$ is conserved if there is a $U$ such that the left-and right-hand sides of Eqs. (2.19) and (2.20) are identical. As a special case of that, if the tensors $Y$ and $Z$ are real, the potential is invariant under a $\mathrm{CP}$ transformation, which we refer to as $C P_{0}$,

$$
U_{a^{\prime}}^{a}=\delta_{a^{\prime}}^{a}
$$

\footnotetext{
${ }^{4}$ This is often referred to as a generalised CP transformation.
} 
$C P_{0}$ is often referred to as trivial or canonical CP.

In doing so, we note that a physical CP transformation will have to treat the two components of an $S U(2)_{L}$ doublet consistently with that symmetry, i.e. both must transform identically under the CP symmetry [76].

In preparation for Section 8, where CPIs for spontaneous CP violation will be constructed, we define how VEVs behave under basis transformations:

$$
\begin{aligned}
v_{a} & \mapsto V_{a}^{a^{\prime}} v_{a^{\prime}}, \\
v^{* a} & \mapsto v^{* a^{\prime}} V_{a^{\prime}}^{\dagger^{a}}
\end{aligned}
$$

where $v \equiv\left(v_{1}, \ldots\right)$ with $v_{i}=\left\langle\varphi_{i}\right\rangle$, and $V$ denotes the transformation matrix of the fields $\phi$. Similarly, under CP transformations, they become

$$
\begin{aligned}
v_{a} & \mapsto v^{* a^{\prime}} U_{a^{\prime}}^{a}, \\
v^{* a} & \mapsto U_{a}^{\dagger^{\prime}} v_{a^{\prime}} .
\end{aligned}
$$

\subsection{CP-odd invariants for explicit CP violation}

In the previous subsection, the standard form for even scalar potentials was introduced and the effects of symmetry transformations, basis transformations and CP transformations has been analysed. This subsection starts with a discussion of simple basis invariants constructed from $Y$ and $Z$ tensors. After that, the general definition of CP-odd basis invariants (CPIs) that contain $Y$ and $Z$ is given.

Finally, the CP properties of such invariants will be analysed. CPIs of this type, that only consist of parameters of the potential and in particular do not contain VEVs, indicate explicit violation of CP. The exact statement is that if all possible CPIs are zero, then the theory is CP conserving. Vice-versa, if at least one CPI is non-zero, the theory violates $\mathrm{CP}$ explicitly. Invariants including VEVs, such that they indicate spontaneous violation of CP, will be introduced in Section 8 .

Although the material of this subsection is not new as such, we present it here in order to make our discussion self contained.

Any product of $Y$ and $Z$ tensors where all indices are correctly contracted forms a basis invariant. Starting with $Y$ and considering $Z$ a little later, the simplest invariant (that is however not CP-odd) is

$$
Y_{a}^{a}
$$

For products of two $Y$ tensors, the only possible contractions are

$$
Y_{a}^{a} Y_{b}^{b} \text { and } Y_{b}^{a} Y_{a}^{b}
$$

The above contractions correspond to the two different permutations of the two upper indices, namely firstly the identity:

$$
Y_{a}^{a} Y_{b}^{b} \Leftrightarrow a \mapsto a \text { and } b \mapsto b,
$$

and secondly the transposition:

$$
Y_{b}^{a} Y_{a}^{b} \Leftrightarrow a \mapsto b \text { and } b \mapsto a .
$$


More formally, one can thus also express all invariants that consist of two $Y$ tensors by

$$
Y_{\sigma(a)}^{a} Y_{\sigma(b)}^{b} \text { with } \sigma \in S_{2}
$$

where $\sigma$ is now one of the two elements of the permutation group $S_{2}$. The invariant built from two $Y$ tensors that corresponds to the identity of $S_{2}$ is the square of the simplest invariant. Thus, only the second invariant is irreducible, which for our purposes will be defined as not being a product or power of smaller invariants.

It is generally true that all possible invariants can be obtained through permutations of indices: all conceivable invariants built from $3 Y$ tensors are given by

$$
Y_{\sigma(a)}^{a} Y_{\sigma(b)}^{b} Y_{\sigma(c)}^{c} \text { with } \sigma \in S_{3}
$$

or explicitly

$$
Y_{a}^{a} Y_{b}^{b} Y_{c}^{c}, Y_{a}^{a} Y_{c}^{b} Y_{b}^{c}, Y_{c}^{a} Y_{b}^{b} Y_{a}^{c}, Y_{b}^{a} Y_{a}^{b} Y_{c}^{c}, Y_{c}^{a} Y_{a}^{b} Y_{b}^{c}, Y_{b}^{a} Y_{c}^{b} Y_{a}^{c}
$$

Here, only the last two invariants are new and irreducible, i.e. not products of smaller invariants. Additionally, they turn out to be equivalent as can be seen by renaming the indices $b \leftrightarrow c$ into each other.

The identification of invariants with elements of permutation groups will be used later to systematically identify all irreducible invariants of a given order. Beyond that, it is this formalism that is going to make it possible to determine which invariants are CP-even and which are not.

But before that, some more examples are in order, as the situation is more complicated for invariants containing $Z$ tensors. There are already two invariants that could be built from a single $Z$ tensor that again correspond to the two possible permutations of positions of the two upper indices:

$$
Z_{\sigma(a) \sigma(b)}^{a b} \text { with } \sigma \in S_{2}
$$

or explicitly:

$$
Z_{a b}^{a b} \text { and } Z_{b a}^{a b} \text {. }
$$

Because the $Z$ tensor of potentials considered here is symmetric under exchanging both upper or both lower indices, cf. below Eq. (2.6), both invariants built from one $Z$ tensor are equivalent. For larger numbers of tensors, the number of permutations grows quickly, however, luckily, many invariants do not need to be considered either because they are products of smaller invariants, or because they are equivalent due to the symmetry of single tensors themselves or the symmetries of the invariant. For example, for two $Z$ tensors, generally all invariants would be given by

$$
Z_{\sigma(a) \sigma(b)}^{a b} Z_{\sigma(c) \sigma(d)}^{c d} \text { with } \sigma \in S_{4}
$$

but the only new invariants can be chosen to be

$$
Z_{b d}^{a b} Z_{a c}^{c d} \text { and } Z_{c d}^{a b} Z_{a b}^{c d}
$$

All other 22 invariants that correspond to the remaining elements of $S_{4}$ are products of smaller invariants or equivalent to the invariants in Eq. (2.36). 
Generally, a basis invariant $I_{\sigma}^{\left(n_{Z}, m_{Y}\right)}$ built from $m_{Y} \mathrm{Y}$ tensors and $n_{Z} Z$ tensors can be written as

$$
I_{\sigma}^{\left(n_{Z}, m_{Y}\right)} \equiv Y_{\sigma\left(a_{1}\right)}^{a_{1}} \ldots Y_{\sigma\left(a_{m_{Y}}\right)}^{a_{m_{Y}}} Z_{\sigma\left(b_{1}\right) \sigma\left(b_{2}\right)}^{b_{1} b_{2}} \ldots Z_{\sigma\left(b_{2 n_{Z}-1}\right) \sigma\left(b_{2 n_{Z}}\right)}^{b_{2 n_{Z}-1} b_{2 n_{Z}}} \text { with } \sigma \in S_{m_{Y}+2 n_{Z}}
$$

Again, $\sigma$ is a permutation of $m_{Y}+2 n_{Z}$ objects, i.e. $\sigma \in S_{m_{Y}+2 n_{Z}}$. However, not all basis invariants are CP-odd, and in fact, all of the examples in Eqs. (2.26)-(2.36) turn out to be CP-even. To be able to make such statements, one needs to know how basis invariants behave under CP. Under a general CP transformation, a coupling tensor is replaced by its complex conjugate multiplied by unitary basis transformations, earlier denoted by $U$. But, as a basis invariant is, by definition, invariant under basis transformations, the $U$ matrices cancel, leaving only the original product of coupling tensors with tensors replaced by their complex conjugates. The complex conjugate of a coupling tensor on the other hand can be obtained by interchanging upper with lower indices, cf. Eqs. (2.14) and (2.15). For the simplest example, $Y_{a}^{a}$, this works out in the following way:

$$
Y_{a}^{a} \stackrel{C P}{\longrightarrow}\left(Y_{a^{\prime \prime}}^{a^{\prime}}\right)^{*} U_{a^{\prime}}^{\dagger}{ }^{a} U_{a}^{a^{\prime \prime}}=\left(Y_{a^{\prime \prime}}^{a^{\prime}}\right)^{*} \delta_{a^{\prime}}^{a^{\prime \prime}}=\left(Y_{a}^{a}\right)^{*}=Y_{a}^{a},
$$

where in the last step Eq. 22.14) was used. As the right-hand side is the CP conjugate of the left-hand side and is identical to the latter, this shows that this invariant is even under CP transformations. Similarly, and using Eqs. (2.14) and (2.15), one can show that the $\mathrm{CP}$ conjugate of a general basis invariant can be obtained by interchanging upper and lower indices:

$$
\begin{aligned}
& I_{\sigma}^{\left(n_{Z}, m_{Y}\right)} \equiv Y_{\sigma\left(a_{1}\right)}^{a_{1}} \ldots Y_{\sigma\left(a_{m_{Y}}\right)}^{a_{m_{Y}}} Z_{\sigma\left(b_{1}\right) \sigma\left(b_{2}\right)}^{b_{1} b_{2}} \ldots Z_{\sigma\left(b_{2 n_{Z}-1}\right) \sigma\left(b_{2 n_{Z}}\right)}^{b_{2 n_{Z}-1} b_{2 n_{Z}}} \\
& \stackrel{C P}{\longrightarrow} Y_{a_{1}}^{\sigma\left(a_{1}\right)} \ldots Y_{a_{m_{Y}}}^{\sigma\left(a_{m_{Y}}\right)} Z_{b_{1} b_{2}}^{\sigma\left(b_{1}\right) \sigma\left(b_{2}\right)} \ldots Z_{b_{2 n_{Z}-1} b_{2 n_{Z}}}^{\sigma\left(b_{2 n_{2}}{ }^{-1}\right) \sigma\left(b_{2 n_{Z}}\right)}=\left[I_{\sigma}^{\left(n_{Z}, m_{Y}\right)}\right]^{*} .
\end{aligned}
$$

If one has found an invariant $I$ that is not CP-even, i.e. that does not equal its $\mathrm{CP}$ conjugate $I^{*}$, one can (as is well-known [24]) extract the CP-odd part by subtracting the CP-conjugated from the original invariant:

$$
\mathcal{I}=I-I^{*}
$$

The above procedure, of tracing over all internal spaces and identifying the remaining imaginary part, is actually more general than discussed above, and it applies also to cases with scalars and fermions [24]. As a CPI is already completely defined by stating half of it, $I$, in the following often $I^{*}$ will be omitted or abbreviated. When $\mathcal{I}$ is given, it is implied that the quantity to follow is the difference between a basis invariant $I$ and its CP conjugate.

For the example invariants in Eqs. (2.26)-(2.36), interchanging upper and lower indices and possibly renaming indices shows that all of them are equal to their $\mathrm{CP}$ conjugate and thus CP-even. For larger invariants, this process can become quite cumbersome. Even worse, in order to show that an invariant is not CP-even, one would have to test all

\footnotetext{
${ }^{5}$ Often, not the full permutation $\sigma$ will be indicated when referring to invariants, but e.g. $I_{2}^{(3,1)}$ would be the second invariant that was found with $n_{Z}=3$ and $m_{Y}=1$.
} 
possible renamings of the indices, which at some point becomes too difficult. Luckily, the symmetry properties of invariants can be analysed and visualised using diagrams that encode which tensors are used and how their indices are contracted with each other. These diagrams are the topic of the next subsection. As the diagrams become more complicated, a more powerful technique relies on analysing so-called contraction matrices that also encode information about the basis invariant and reveal whether it is a CPI. We heavily rely on the contraction matrices for our systematic searches that revealed many new CPIs. As the discussion is somewhat technical, the details concerning contraction matrices are relegated to Appendix A.

\subsection{Diagrams for invariants}

Any basis invariant consisting of contractions of $Y$ and $Z$ can be expressed by a diagram [26]. We use a slightly different notation from the one present in [26]. For each $Y$ or $Z$ draw a vertex and for any contraction of an upper index on a tensor with a lower index of a tensor draw an arrow connecting the vertices corresponding to the tensors. With $X=Y, Z$, the only rule for drawing diagrams is

$$
X_{. .}^{a .} X_{a .}=\bullet \longrightarrow
$$

Additionally, as $Z_{c d}^{a b}$ is symmetric under exchange of $a \leftrightarrow b$ and/or $c \leftrightarrow d$, two lines can be attached to a vertex corresponding to a $Z$ tensor without having to distinguish them in the diagram:

$$
Z_{. .}^{a b} Z_{a b}=\bigodot
$$

Contracting two indices on the same tensor with each other produces a loop:

$$
X_{a .}^{a .}=\bigodot_{0}
$$

Diagrams drawn following these rules make it possible to check if an invariant is CP-even: from Eq. 2.39 follows that the CP conjugate of an invariant produces exactly the same diagram but with inverted directions of arrows as all upper indices have been turned into lower indices and vice versa. An invariant is identical to its $\mathrm{CP}$ conjugate, i.e. CP-even, if the diagrams of the invariant and its $\mathrm{CP}$ conjugate are identical up to the positions of the vertices. The reason for this is that in a product of $Y$ and $Z$ tensors, their position in the product is arbitrary and thus also the position of vertices (except for the type of tensor) $]^{6}$

A few small example diagrams for small invariants mentioned earlier in the text are shown in Figure 1. One can see there that for each of them, inverting the direction of the arrows produces the same diagram and thus the same invariant. This is the case for all of the small examples in Eqs. (2.26)-(2.36). The simplest invariant, $Y_{a}^{a}$ produces the diagram in Eq. (2.43).

\footnotetext{
${ }^{6}$ The internal symmetry of $Z_{c d}^{a b}$ under $a \leftrightarrow b$ and/or $c \leftrightarrow d$ is taken into account by Eq. 2.42.
} 


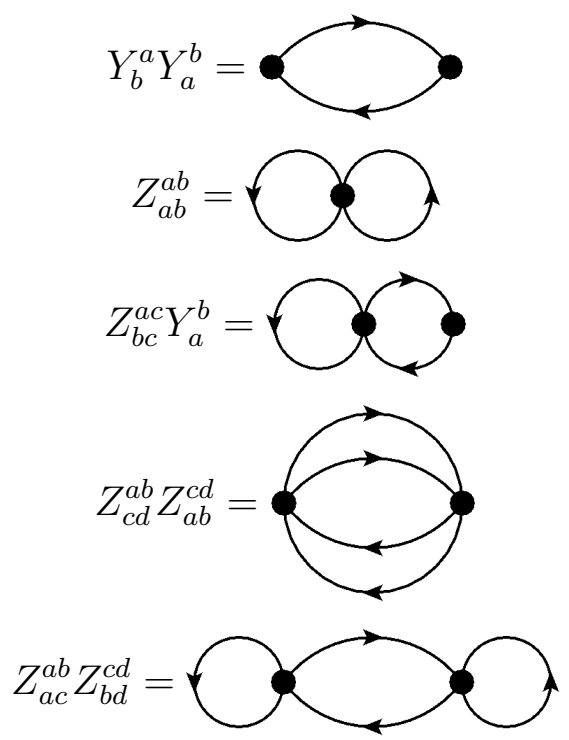

Figure 1: Example diagrams corresponding to small invariants.

All invariants discussed so far were CP-even. The smallest CP-odd invariant was already found in [25] and is given by the difference $\mathcal{I}_{1}=I_{1}-I_{1}^{*}$ of

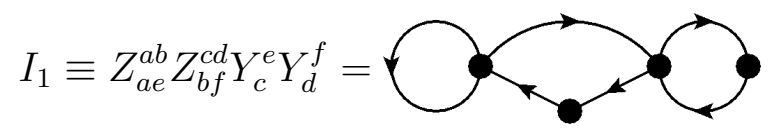

and its CP conjugate

$$
I_{1}^{*} \equiv Z_{a b}^{a e} Z_{c d}^{b f} Y_{e}^{c} Y_{f}^{d}=C .
$$

In whatever ways one tries to interchange the positions of vertices and arrows, it is impossible to make the diagrams equivalent.

Additionally, out of all possible contractions of coupling tensors, many will be related by interchanging the positions of tensors. The symmetries of the diagrams can be used to classify invariants and search for CPIs in a systematic way as will be explained in Appendix A. The results of this systematic search are listed in the following.

\subsection{CP-odd invariants only built from $Z$ tensors}

It is interesting to consider invariants that are only built from $Z$ tensors, as these indicate $\mathrm{CP}$ violation that is mediated purely through the interaction of fields and does not e.g. depend on a mass splitting. One could now wonder if for a non-diagonal $Y$ tensor CP violating effects could be shifted between $Y$ and $Z$ by diagonalising $Y$. However, because this is just another basis change, it drops out in any basis invariants, including also CPIs.

In Appendix B, we list the representative CPIs with up to $n_{Z}=6 Z$ tensors. All other $\mathrm{CPIs}$ are related to these representatives by symmetries or $\mathrm{CP}$ conjugation. 
An important first result is that all invariants up to $n_{Z}=4$ are CP-even. For $n_{Z}=5$, three different CPIs exist:
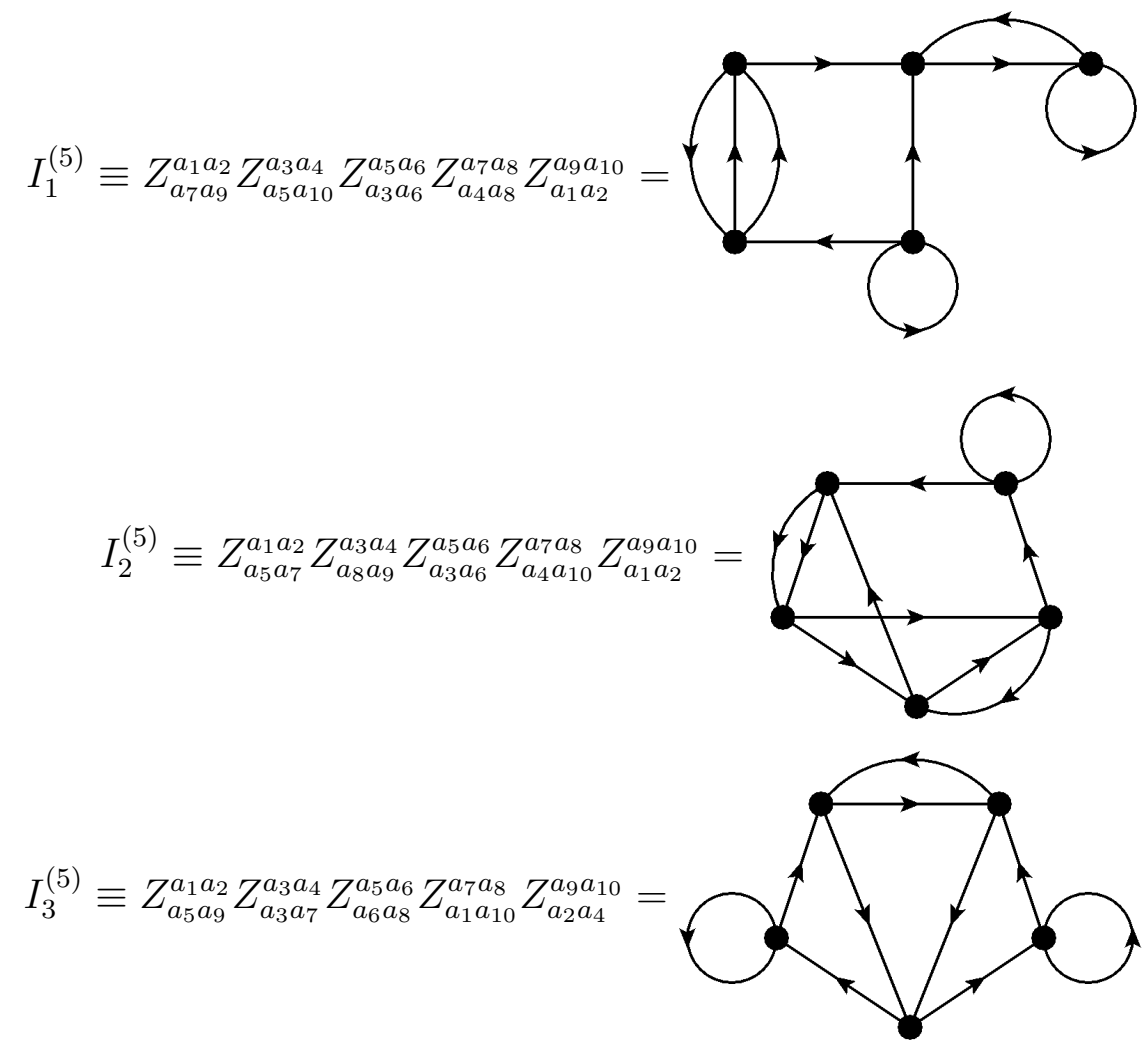

For $n_{Z}=6$, in total 56 different invariants exist out of which three are products of the $n_{Z}=5$ invariants with a completely self-contracted $Z$ tensor, $Z_{a b}^{a b}$. These will not provide any new information. Next, of particular interest are those invariants that contain no selfloops, as we found that invariants with self-loops, i.e. $Z_{a}^{a}$. often vanish for the example potentials considered in this work. With $n_{Z}=6$, only 5 invariants without self-loops remain:

$$
\begin{aligned}
I_{1}^{(6)} & \equiv Z_{a_{11} a_{10}}^{a_{1} a_{2}} Z_{a_{5} a_{8}}^{a_{3} a_{4}} Z_{a_{7} a_{12}}^{a_{5} a_{6}} Z_{a_{9} a_{6}}^{a_{7} a_{8}} Z_{a_{3} a_{4}}^{a_{9} a_{10}} Z_{a_{1} a_{2}}^{a_{11} a_{12}} \\
I_{2}^{(6)} & \equiv Z_{a_{7} a_{10}}^{a_{1} a_{2}} Z_{a_{11} a_{6}}^{a_{3} a_{4}} Z_{a_{9} a_{8}}^{a_{5} a_{6}} Z_{a_{3} a_{12}}^{a_{7} a_{8}} Z_{a_{5} a_{4}}^{a_{9} a_{10}} Z_{a_{1} a_{2}}^{a_{11} a_{12}} \\
I_{3}^{(6)} & \equiv Z_{a_{7} a_{10}}^{a_{1} a_{2}} Z_{a_{9} a_{6}}^{a_{3} a_{4}} Z_{a_{11} a_{8}}^{a_{5} a_{6}} Z_{a_{3} a_{12}}^{a_{7} a_{8}} Z_{a_{5} a_{4}}^{a_{9} a_{10}} Z_{a_{1} a_{2}}^{a_{11} a_{12}} \\
I_{4}^{(6)} & \equiv Z_{a_{11} a_{10}}^{a_{1} a_{2}} Z_{a_{5} a_{8}}^{a_{3} a_{4}} Z_{a_{7} a_{12}}^{a_{5} a_{6}} Z_{a_{9} a_{6}}^{a_{7} a_{8}} Z_{a_{1} a_{4}}^{a_{9} a_{10}} Z_{a_{3} a_{2}}^{a_{11} a_{12}} \\
I_{5}^{(6)} & \equiv Z_{a_{7} a_{12}}^{a_{1} a_{2}} Z_{a_{5} a_{10}}^{a_{3} a_{4}} Z_{a_{9} a_{8}}^{a_{5} a_{6}} Z_{a_{11} a_{4}}^{a_{7} a_{8}} Z_{a_{1} a_{6}}^{a_{9} a_{10}} Z_{a_{3} a_{2}}^{a_{11} a_{12}} .
\end{aligned}
$$

The diagrams that correspond to the above invariants with $n_{Z}=6$ and the remaining representative CPIs with up to $n_{Z}=6$ are listed in Appendix B. 


\subsection{CP-odd invariants built from $Y$ and $Z$ tensors}

Mixed invariants consisting of $Y$ and $Z$ tensors can be CP-odd at lower numbers of $Z$ tensors than $n_{Z}=5$. The reason for this is that additional asymmetries can be introduced in the diagrams. The smallest CPI found in 25], Eqs. (2.44) and (2.45), is of this type with $m_{Y}=2, n_{Z}=2$ and will not be repeated here. There are no other CPIs for $m_{Y}=2, n_{Z}=2$ that are not equivalent to the aforementioned one. The next smallest CPIs are found for $n_{Z}=3$ and $m_{Y}=1$. There are two different classes with the following representatives:

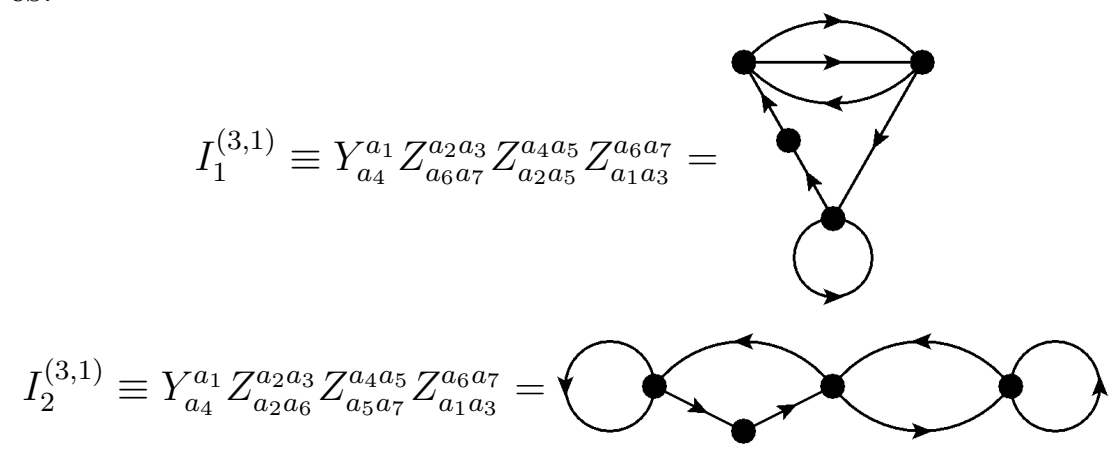

However, both invariants in Eqs. 2.54) and 2.55) contain self-loops. As these often vanish in examples, one would preferably like to find invariants without self-loops. Such invariants can already be found for $n_{Z}=3, m_{Y}=2$. There are in total 13 invariants with this number of $Y$ and $Z$ tensors, out of which 2 have no $Z$-self-loops. In one of them, the $Y$ tensor is inserted in a $Z$ loop and will only make a difference if $Y$ is not proportional to the identity, while the other invariant has genuinely no $Z$-self-loops. These invariants and diagrams are

$$
I_{1}^{(3,2)} \equiv Y_{a_{7}}^{a_{1}} Y_{a_{5}}^{a_{2}} Z_{a_{6} a_{7}}^{a_{3} a_{4}} Z_{a_{3} a_{4}}^{a_{5} a_{6}} Z_{a_{1} a_{2}}^{a_{7} a_{8}}=
$$

Naively, there are 53 classes of invariants with $m_{Y}=3, n_{Z}=3$. However, many of these will be products of smaller CPIs with small CP-even invariants. Eventually, there are 10 invariants without $Z$-self loops which are not products of smaller invariants, the representatives of which are listed in the following:

$$
\begin{gathered}
I_{1}^{(3,3)} \equiv Y_{a_{8}}^{a_{1}} Y_{a_{6}}^{a_{2}} Y_{a_{4}}^{a_{3}} Z_{a_{7} a_{9}}^{a_{4} a_{5}} Z_{a_{3} a_{5}}^{a_{6} a_{7}} Z_{a_{1} a_{2}}^{a_{8} a_{9}}, \\
I_{2}^{(3,3)} \equiv Y_{a_{6}}^{a_{1}} Y_{a_{7}}^{a_{2}} Y_{a_{4}}^{a_{3}} Z_{a_{8} a_{9}}^{a_{4} a_{5}} Z_{a_{3} a_{5}}^{a_{6} a_{7}} Z_{a_{1} a_{2}}^{a_{8} a_{9}},
\end{gathered}
$$




$$
\begin{gathered}
I_{3}^{(3,3)} \equiv Y_{a_{8}}^{a_{1}} Y_{a_{4}}^{a_{2}} Y_{a_{6}}^{a_{3}} Z_{a_{7} a_{9}}^{a_{4} a_{5}} Z_{a_{3} a_{5}}^{a_{6} a_{7}} Z_{a_{1} a_{2}}^{a_{8} a_{9}} \\
I_{4}^{(3,3)} \equiv Y_{a_{6}}^{a_{1}} Y_{a_{4}}^{a_{2}} Y_{a_{8}}^{a_{3}} Z_{a_{7} a_{9}}^{a_{4} a_{5}} Z_{a_{3} a_{5}}^{a_{6} a_{7}} Z_{a_{1} a_{2}}^{a_{8} a_{9}} \\
I_{5}^{(3,3)} \equiv Y_{a_{6}}^{a_{1}} Y_{a_{4}}^{a_{2}} Y_{a_{7}}^{a_{3}} Z_{a_{8} a_{9}}^{a_{4} a_{5}} Z_{a_{3} a_{5}}^{a_{6} a_{7}} Z_{a_{1} a_{2}}^{a_{8} a_{9}} \\
I_{6}^{(3,3)} \equiv Y_{a_{8}}^{a_{1}} Y_{a_{3}}^{a_{2}} Y_{a_{6}}^{a_{3}} Z_{a_{7} a_{9}}^{a_{4} a_{5}} Z_{a_{4} a_{5}}^{a_{6} a_{7}} Z_{a_{1} a_{2}}^{a_{8} a_{9}} \\
I_{7}^{(3,3)} \equiv Y_{a_{6}}^{a_{1}} Y_{a_{3}}^{a_{2}} Y_{a_{8}}^{a_{3}} Z_{a_{7} a_{9}}^{a_{4} a_{5}} Z_{a_{4} a_{5}}^{a_{6} a_{7}} Z_{a_{1} a_{2}}^{a_{8} a_{9}} \\
I_{8}^{(3,3)} \equiv Y_{a_{6}}^{a_{1}} Y_{a_{3}}^{a_{2}} Y_{a_{4}}^{a_{3}} Z_{a_{7} a_{8}}^{a_{4} a_{5}} Z_{a_{5} a_{9}}^{a_{6} a_{7}} Z_{a_{1} a_{2}}^{a_{8} a_{9}} \\
I_{9}^{(3,3)} \equiv Y_{a_{6}}^{a_{1}} Y_{a_{3}}^{a_{2}} Y_{a_{4}}^{a_{3}} Z_{a_{7} a_{8}}^{a_{4} a_{5}} Z_{a_{1} a_{9}}^{a_{6} a_{7}} Z_{a_{2} a_{5}}^{a_{3} a_{9}} \\
I_{10}^{(3,3)} \equiv Y_{a_{6}}^{a_{1}} Y_{a_{3}}^{a_{2}} Y_{a_{4}}^{a_{3}} Z_{a_{8} a_{9}}^{a_{4} a_{5}} Z_{a_{1} a_{5}}^{a_{6} a_{7}} Z_{a_{2} a_{7}}^{a_{8} a_{9}}
\end{gathered}
$$

Finally, we have also analysed invariants with $n_{Z}=4$ and $m_{Y}=1$. Naively, there are 18 different invariants with this number of $Z$ and $Y$ tensors. However, there is only one invariant with this number of coupling tensors without self-loops,

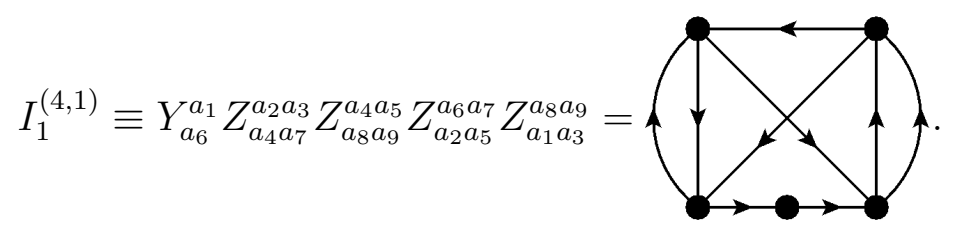

This concludes our list of CPIs for explicit CP violation used in the main text of this paper. Following our systematic approach, we have also calculated larger invariants and the obtained CPIs are collected in Appendix B.

\section{Two Higgs doublet model potential}

As a first example for an application of CPIs that is well known in the literature we consider the most general potential of two copies of SM Higgs bosons. For this potential, a complete basis of CPIs is known [27]. All of these four CPIs have also been produced in our systematic search. Using a slightly modified version of the notation in [25], the general 2HDM potential takes the form

$$
\begin{aligned}
V\left(H_{1}, H_{2}\right)= & m_{1}^{2} H_{1}^{\dagger} H_{1}+m_{12}^{2} e^{i \theta_{0}} H_{1}^{\dagger} H_{2}+m_{12}^{2} e^{-i \theta_{0}} H_{2}^{\dagger} H_{1}+m_{2}^{2} H_{2}^{\dagger} H_{2}+ \\
& +a_{1}\left(H_{1}^{\dagger} H_{1}\right)^{2}+a_{2}\left(H_{2}^{\dagger} H_{2}\right)^{2} \\
& +b\left(H_{1}^{\dagger} H_{1}\right)\left(H_{2}^{\dagger} H_{2}\right)+b^{\prime}\left(H_{1}^{\dagger} H_{2}\right)\left(H_{2}^{\dagger} H_{1}\right)+ \\
& +c_{1} e^{i \theta_{1}}\left(H_{1}^{\dagger} H_{1}\right)\left(H_{2}^{\dagger} H_{1}\right)+c_{1} e^{-i \theta_{1}}\left(H_{1}^{\dagger} H_{1}\right)\left(H_{1}^{\dagger} H_{2}\right)+ \\
& +c_{2} e^{i \theta_{2}}\left(H_{2}^{\dagger} H_{2}\right)\left(H_{2}^{\dagger} H_{1}\right)+c_{2} e^{-i \theta_{2}}\left(H_{2}^{\dagger} H_{2}\right)\left(H_{1}^{\dagger} H_{2}\right)+ \\
& +d e^{i \theta_{3}}\left(H_{1}^{\dagger} H_{2}\right)^{2}+d e^{-i \theta_{3}}\left(H_{2}^{\dagger} H_{1}\right)^{2} .
\end{aligned}
$$


Here $H_{1}=\left(h_{1,1}, h_{1,2}\right)$ and $H_{2}=\left(h_{2,1}, h_{2,2}\right)$ and the $S U(2)_{L}$ invariant contractions are indicated by the brackets e.g. $\left(H_{1}^{\dagger} H_{1}\right)^{2}=\left(h_{1,1}^{\dagger} h_{1,1}+h_{1,2}^{\dagger} h_{1,2}\right)^{2}$. Eq. (2.2) becomes

$$
\phi=\left(\varphi_{1}, \varphi_{2}, \varphi_{3}, \varphi_{4}\right)=\left(h_{1,1}, h_{1,2}, h_{2,1}, h_{2,2}\right),
$$

such that the $Z$ tensor corresponding to the quartic terms of the scalar potential has $4^{4}=256$ components. It is straigtforward to determine these explicitly for the potential of Eq. (3.1). In the following, we display the non-vanishing ones,

$$
\begin{aligned}
& Z_{11}^{11}=Z_{22}^{22}=2 Z_{12}^{12}=a_{1}, \\
& Z_{33}^{33}=Z_{44}^{44}=2 Z_{34}^{34}=a_{2}, \\
& 4 Z_{14}^{14}=4 Z_{23}^{23}=b,=Z^{23}=b^{\prime}, \\
& 4 Z_{23}^{14}=4 Z_{13}^{13}=4 Z_{24}^{24}=b+b^{\prime}, \\
& 4 Z_{14}^{12}=4 Z_{23}^{12}=2 Z_{13}^{11}=2 Z_{24}^{22}=c_{1} e^{i \theta_{1}}, \\
& 4 Z_{34}^{14}=4 Z_{34}^{23}=2 Z_{33}^{13}=2 Z_{44}^{24}=c_{2} e^{i \theta_{2}}, \\
& 2 Z_{12}^{34}=Z_{11}^{33}=Z_{22}^{44}=d e^{i \theta_{3}},
\end{aligned}
$$

and remind the reader of the general relations $Z_{c d}^{a b}=Z_{c d}^{b a}=Z_{d c}^{a b}=Z_{d c}^{b a}$ and $Z_{a b}^{c d}=\left(Z_{c d}^{a b}\right)^{*}$. Having determined the $Z$ tensor in terms of the parameters of the potential, we can calculate CPIs explicitly.

As a first illustration, we show the results of CPIs for the two Higgs doublet potential of Eq. (3.1). In our notation, the smallest one becomes

$$
\begin{aligned}
\mathcal{I}_{1}= & -9 i m_{12}^{2}\left(m_{1}^{2}-m_{2}^{2}\right)\left[c_{2}\left(2 a_{1}-b-b^{\prime}\right) \sin \left(\theta_{0}+\theta_{2}\right)+c_{1}\left(2 a_{2}-b-b^{\prime}\right) \sin \left(\theta_{0}+\theta_{1}\right)\right. \\
& \left.+2 d\left(c_{1} \sin \left(\theta_{0}-\theta_{1}-\theta_{3}\right)+c_{2} \sin \left(\theta_{0}-\theta_{2}-\theta_{3}\right)\right)\right] \\
& -9 i m_{12}^{4}\left[4 d\left(a_{2}-a_{1}\right) \sin \left(2 \theta_{0}-\theta_{3}\right)+c_{1}^{2} \sin \left(2\left(\theta_{0}+\theta_{1}\right)\right)\right. \\
& \left.-c_{2}\left(2 c_{1} \sin \left(\theta_{1}-\theta_{2}\right)+c_{2} \sin \left(2\left(\theta_{0}+\theta_{2}\right)\right)\right)\right] \\
& -9 i c_{1} c_{2}\left(m_{1}^{2}-m_{2}^{2}\right)^{2} \sin \left(\theta_{1}-\theta_{2}\right) .
\end{aligned}
$$

There are many ways of setting this expression to zero, the simpler ones involve $m_{12}^{2}=0$ which leaves only the last line in the expression, which vanishes either for $m_{1}^{2}-m_{2}^{2}$ or $\sin \left(\theta_{1}-\theta_{2}\right)$. Alternatively, if $m_{1}^{2}-m_{2}^{2}=0$ there are other combinations of constraints that make this CPI vanish, including $\sin \left(2 \theta_{0}-\theta_{3}\right)=\sin \left(2\left(\theta_{0}+\theta_{1}\right)\right)=0$. However, at this stage it is not clear if any of these constraints are sufficient to guarantee conservation of $\mathrm{CP}$, as other CPIs could still be non-zero. The invariant $\mathcal{I}_{1}$ being non-zero always requires $m_{12} \neq 0$ or $m_{1}^{2} \neq m_{2}^{2}$.

As already mentioned, a complete basis of CPIs is known for the 2HDM potential, cf. [27]. Of the four invariants given in that paper, three are equivalent to invariants given in Section 2 of our paper (GH denotes the invariant in [27]):

$$
I_{2 Y 2 Z}^{(\mathrm{GH})}=I_{1}=I_{1}^{(2,2)},
$$




$$
I_{Y 3 Z}^{(\mathrm{GH})}=I_{2}^{(3,1)},
$$

and

$$
I_{3 Y 3 Z}^{(\mathrm{GH})}=\left(I_{5}^{(3,3)}\right)^{*} .
$$

The fourth invariant listed in [27] has $n_{Z}=6$ and has not been given here yet as it contains $Z$-self-loops. For completeness, we present it here following our general notation as well as diagrammatically:

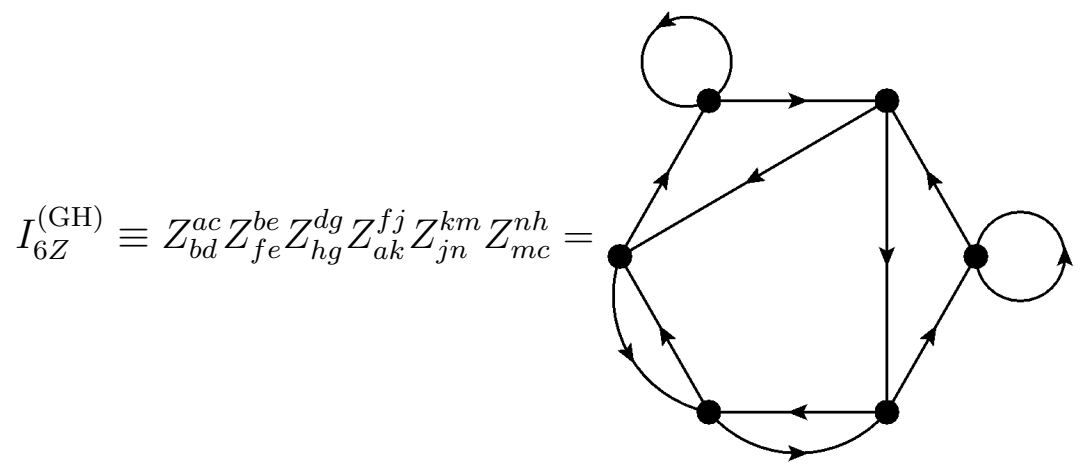

All CP-odd invariants with $5 Z$ tensors, cf. Eqs. (2.46)-(2.48), vanish for this potential. For this potential, $Z_{a c}^{a b}$ is non-diagonal, which is why the CPIs found in [27] produce interesting results. While the invariants from 27] form a complete basis of CPIs for the $2 \mathrm{HDM}$, all of them are zero for the potentials considered in the remainder of this paper. It is our systematic search that reveals new non-zero CPIs in those situations.

\section{$4 \quad A_{4}=\Delta(12)$ invariant potentials}

In this section we study potentials invariant under the discrete group $A_{4}$. We start with a field content of a single triplet of SM singlets, then consider a triplet of $S U(2)_{L}$ doublets, two triplets of SM singlets and two triplets of $S U(2)_{L}$ doublets.

$A_{4}$ contains a real triplet and three one-dimensional representations. The product of two triplets decomposes as

$$
\mathbf{3} \otimes \mathbf{3}=\left(\mathbf{1}_{0}+\mathbf{1}_{1}+\mathbf{1}_{2}+\mathbf{3}\right)_{s}+\mathbf{3}_{a} .
$$

Symmetric and antisymmetric combinations are denoted by subscripts $s$ and $a$, respectively. Throughout this section we work in the basis of [77] which can be easily generalised to $\Delta(27)$ and the complete $\Delta\left(3 n^{2}\right)$ series [12,78] studied in the Sections 5 and 6 .

\subsection{One flavour triplet}

With one triplet field, only the symmetric contribution in Eq. (4.1) matters. It is convenient to define

$$
V_{0}(\varphi)=-m_{\varphi}^{2} \sum_{i} \varphi_{i} \varphi^{* i}+r\left(\sum_{i} \varphi_{i} \varphi^{* i}\right)^{2}+s \sum_{i}\left(\varphi_{i} \varphi^{* i}\right)^{2}
$$


where one notes that the first two terms are $S U(3)$ invariant. We consider $\varphi$ to be additionally charged under some $U(1)$ symmetry (or an appropriate discrete subgroup) such that terms of the form $\varphi_{i} \varphi_{i}$ or $\varphi_{i} \varphi_{i} \varphi_{i}$, for example, are not allowed. This leads to a more direct generalisation of the case where the SM gauge group applies.

The resulting renormalisable scalar potential for $A_{4}$ reads

$$
\begin{aligned}
V_{A_{4}}(\varphi)=V_{0}(\varphi) & +c\left(\varphi_{1} \varphi_{1} \varphi^{* 3} \varphi^{* 3}+\varphi_{2} \varphi_{2} \varphi^{* 1} \varphi^{* 1}+\varphi_{3} \varphi_{3} \varphi^{* 2} \varphi^{* 2}\right) \\
& +c^{*}\left(\varphi^{* 1} \varphi^{* 1} \varphi_{3} \varphi_{3}+\varphi^{* 2} \varphi^{* 2} \varphi_{1} \varphi_{1}+\varphi^{* 3} \varphi^{* 3} \varphi_{2} \varphi_{2}\right)
\end{aligned}
$$

noting that this includes, as expected, four independent quartic terms. Henceforth we use the convenient abbreviations cycl. to denote the cyclic permutations, and h.c. to indicate the hermitian conjugate. We thus write the $A_{4}$ invariant potential of Eq. (4.3) in the compact form:

$$
V_{A_{4}}(\varphi)=V_{0}(\varphi)+\left[c\left(\varphi_{1} \varphi_{1} \varphi^{* 3} \varphi^{* 3}+\text { cycl. }\right)+\text { h.c. }\right] .
$$

The $A_{4}$ symmetric potential respects the general CP symmetry with a 2-3 swap, namely the CP symmetry with unitary matrix $U_{23}$

$$
U_{23}=\left(\begin{array}{lll}
1 & 0 & 0 \\
0 & 0 & 1 \\
0 & 1 & 0
\end{array}\right)
$$

for arbitrary coefficients $r, s \in \mathbb{R}$ and $c \in \mathbb{C}$. Hence, despite the occurrence of the complex coupling $c$ the $A_{4}$ symmetric potential of one triplet is invariant under this general CP symmetry. For this reason, all possible CPIs for this potential will be zero.

\subsection{One flavour triplet of Higgs doublets}

If each component of the $A_{4}$ triplet is an $S U(2)_{L}$ doublet,

$$
H=\left(h_{1 \alpha}, h_{2 \beta}, h_{3 \gamma}\right)
$$

the potential is rather similar to the previous case. Indeed there is one additional invariant, due to the two different ways to perform the $S U(2)_{L}$ contraction on the $A_{4}$ invariant $\left(\sum_{i} \varphi_{i} \varphi^{* i}\right)^{2}$, when the $\varphi$ are replaced by Higgs doublets 7

$$
\sum_{i, j, \alpha, \beta}\left[r_{1}\left(h_{i \alpha} h^{* i \alpha}\right)\left(h_{j \beta} h^{* j \beta}\right)+r_{2}\left(h_{i \alpha} h^{* i \beta}\right)\left(h_{j \beta} h^{* j \alpha}\right)\right] \text {. }
$$

Here we highlight the $S U(2)_{L}$ indices to clarify the distinct $S U(2)_{L}$ contractions. We define $V_{0}(H)$ in analogy with Eq. 4.2):

\footnotetext{
${ }^{7}$ Since the doublet 2 of $S U(2)_{L}$ is a pseudoreal representation, it is also possible to combine $\left(h_{i \alpha} h_{j \beta} \epsilon^{\alpha \beta}\right)\left(h^{* i \gamma} h^{* j \delta} \epsilon_{\gamma \delta}\right)$ using the antisymmetric $\epsilon$ tensor. However, such a term is not linearly independent of the two terms in Eq. 4.7) as can be easily seen in an explicit calculation or by noting that $\mathbf{2} \times \mathbf{2}=\mathbf{1}+\mathbf{3}$ which entails only two independent $S U(2)_{L}$ invariant quartic terms.
} 


$$
\begin{aligned}
V_{0}(H)= & -m_{h}^{2} \sum_{i, \alpha} h_{i \alpha} h^{* i \alpha}+\sum_{i, j, \alpha, \beta}\left[r_{1}\left(h_{i \alpha} h^{* i \alpha}\right)\left(h_{j \beta} h^{* j \beta}\right)+r_{2}\left(h_{i \alpha} h^{* i \beta}\right)\left(h_{j \beta} h^{* j \alpha}\right)\right] \\
& +s \sum_{i, \alpha, \beta}\left(h_{i \alpha} h^{* i \alpha}\right)\left(h_{i \beta} h^{* i \beta}\right)
\end{aligned}
$$

and the $A_{4}$ potential is then

$$
V_{A_{4}}(H)=V_{0}(H)+\sum_{\alpha, \beta}\left[c\left(h_{1 \alpha} h_{1 \beta} h^{* 3 \alpha} h^{* 3 \beta}+\text { cycl. }\right)+\text { h.c. }\right] .
$$

This potential is also invariant under a CP transformation that involves swapping the second and third component in flavour space while keeping $S U(2)_{L}$ contractions unchanged, i.e. $h_{2 \alpha} \rightarrow h^{* 3 \alpha}$ etc.:

$$
U_{23}^{H}=\left(\begin{array}{ccc}
1 & 0 & 0 \\
0 & 0 & 1 \\
0 & 1 & 0
\end{array}\right) \otimes \delta_{\beta}^{\alpha}
$$

Therefore, CP is conserved automatically for this potential and all possible CPIs necessarily vanish.

\subsection{Two flavour triplets}

Typically, realistic models of flavour require more than just one triplet flavon. We therefore consider the potential involving two physically different flavon fields $\varphi$ and $\varphi^{\prime}$ which both transform in the triplet representation of $A_{4}$. In the case of two $A_{4}$ triplets distinguished by additional symmetries so that the total symmetry is $A_{4} \times U(1) \times U(1)^{\prime}$, the potential includes a total of seven independent mixed quartic invariants of the form $\varphi \varphi^{\prime} \varphi^{*} \varphi^{*}$. It is convenient to define:

$$
\begin{aligned}
V_{1}\left(\varphi, \varphi^{\prime}\right)= & +\tilde{r}_{1}\left(\sum_{i} \varphi_{i} \varphi^{* i}\right)\left(\sum_{j} \varphi_{j}^{\prime} \varphi^{\prime * j}\right)+\tilde{r}_{2}\left(\sum_{i} \varphi_{i} \varphi^{\prime * i}\right)\left(\sum_{j} \varphi_{j}^{\prime} \varphi^{* j}\right) \\
& +\tilde{s}_{1} \sum_{i}\left(\varphi_{i} \varphi^{* i} \varphi_{i}^{\prime} \varphi^{\prime * i}\right) \\
& +\tilde{s}_{2}\left(\varphi_{1} \varphi^{* 1} \varphi_{2}^{\prime} \varphi^{\prime * 2}+\varphi_{2} \varphi^{* 2} \varphi_{3}^{\prime} \varphi^{\prime * 3}+\varphi_{3} \varphi^{* 3} \varphi_{1}^{\prime} \varphi^{\prime * 1}\right) \\
& +i \tilde{s}_{3}\left[\left(\varphi_{1} \varphi^{\prime * 1} \varphi_{2}^{\prime} \varphi^{* 2}+\text { cycl. }\right)-\left(\varphi^{* 1} \varphi_{1}^{\prime} \varphi^{\prime * 2} \varphi_{2}+\text { cycl. }\right)\right] .
\end{aligned}
$$

Note that in this definition, the term multiplied by $\tilde{r}_{1}$ contains the term multiplied by $\tilde{s}_{2}$ as well as the term obtained from the latter by interchanging $\varphi$ with $\varphi^{\prime}$ :

$$
\left(\varphi_{1}^{\prime} \varphi^{\prime * 1} \varphi_{2} \varphi^{* 2}+\varphi_{2}^{\prime} \varphi^{* 2} \varphi_{3} \varphi^{* 3}+\varphi_{3}^{\prime} \varphi^{\prime * 3} \varphi_{1} \varphi^{* 1}\right)
$$


which is not included separately in $\tilde{s}_{2}$.

The $A_{4}$ symmetric renormalisable potential takes the following explicit form, with $V_{0}$ as defined in Eq. (4.2),

$$
\begin{aligned}
V_{A_{4}}\left(\varphi, \varphi^{\prime}\right)= & V_{0}(\varphi)+V_{0}^{\prime}\left(\varphi^{\prime}\right)+V_{1}\left(\varphi, \varphi^{\prime}\right)+ \\
& +\left[c\left(\varphi_{1} \varphi_{1} \varphi^{* 3} \varphi^{* 3}+\text { cycl. }\right)+\text { h.c. }\right]+\left[c^{\prime}\left(\varphi_{1}^{\prime} \varphi_{1}^{\prime} \varphi^{\prime * 3} \varphi^{\prime * 3}+\text { cycl. }\right)+\text { h.c. }\right] \\
& +\left[\tilde{c}\left(\varphi_{1} \varphi_{1}^{\prime} \varphi^{* 3} \varphi^{\prime * 3}+\text { cycl. }\right)+\text { h.c. }\right]
\end{aligned}
$$

where $V_{0}^{\prime}\left(\varphi^{\prime}\right)$ has the same functional form as $V_{0}(\varphi)$ with different coefficients $m_{\varphi^{\prime}}^{\prime}, r^{\prime}, s^{\prime}$ and depends on $\varphi^{\prime}$.

Unlike the previous $A_{4}$ invariant potentials, this potential in general violates $\mathrm{CP}$, as confirmed by the non-zero CPIs listed in Table 1 of Section 7. The expressions are cumbersome and we do not reproduce them here. The non-vanishing $\operatorname{CPIs} \mathcal{I}_{2}^{(6)}, \mathcal{I}_{3}^{(6)}, \mathcal{I}_{4}^{(6)}, \mathcal{I}_{5}^{(6)}$ (Eqs. 2.50 2.51 2.52 2.53) all factorise as a product of $\tilde{s}_{2}$ with different complicated functions of the remaining parameters, for example, $\mathcal{I}_{2}^{(6)}$ takes the form:

$$
\mathcal{I}_{2}^{(6)}=\tilde{s}_{2} f(\ldots)
$$

where $f$ is a complicated function of the other parameters. Such a dependence on $\tilde{s}_{2}$ is expected because it corresponds to a CP symmetry, where one imposes $U_{23}$ of Eq. (4.5) on both triplets, corresponding to the block matrix:

$$
U_{23}^{\varphi \varphi^{\prime}}=\left(\begin{array}{cc}
U_{23} & 0 \\
0 & U_{23}
\end{array}\right) .
$$

This CP symmetry constrains the potential such that $\tilde{s}_{2}=0$, which forces all CPIs to vanish as expected from the presence of a CP symmetry. Furthermore, applying instead the trivial CP symmetry $C P_{0}$ forces $\tilde{s}_{3}=0$ and all other complex parameters $\left(c, c^{\prime}, \tilde{c}\right)$ to be real. As expected, this renders $f(\ldots)=0$ in Eq. (4.14), an makes all other CPIs vanish as well.

\subsection{Two flavour triplets of Higgs doublets}

Earlier, when considering a potential of an $A_{4}$ triplet of $S U(2)_{L}$ doublets, the only difference was that the term with coefficient $r$ split into two different invariants corresponding to two different possible $S U(2)_{L}$ contractions, cf. Eq. (4.7). Similarly, the potential of two triplets of SM doublets:

$$
H=\left(h_{1 \alpha}, h_{2 \beta}, h_{3 \gamma}\right), \quad H^{\prime}=\left(h_{1 \alpha}^{\prime}, h_{2 \beta}^{\prime}, h_{3 \gamma}^{\prime}\right)
$$

can be obtained from the corresponding potential of singlets, Eq. (4.13). In the first two parts of the potential, $V_{0}(\varphi)$ and $V_{0}\left(\varphi^{\prime}\right)$, as earlier, there are two different ways of $S U(2)_{L}$-contracting the invariants with coefficients $r$ and $r^{\prime}$. In the part of the potential with $A_{4}$ contractions as in $V_{1}\left(\varphi, \varphi^{\prime}\right)$, for all $A_{4}$ invariants two possible ways of $S U(2)_{L}$ 
contracting the fields exists and this part of the potential becomes

$$
\begin{aligned}
V_{1}\left(H, H^{\prime}\right) & =\sum_{i, j, \alpha, \beta}\left[\tilde{r}_{11} h_{i \alpha} h^{* i \alpha} h_{j \beta}^{\prime} h^{\prime * j \beta}+\tilde{r}_{12} h_{i \alpha} h^{\prime * j \alpha} h_{j \beta}^{\prime} h^{* i \beta}\right] \\
& +\sum_{i, j, \alpha, \beta}\left[\tilde{r}_{21} h_{i \alpha} h^{\prime * i \alpha} h_{j \beta}^{\prime} h^{* j \beta}+\tilde{r}_{22} h_{i \alpha} h^{* j \alpha} h_{j \beta}^{\prime} h^{\prime * i \beta}\right] \\
& +\sum_{i, \alpha, \beta}\left[\tilde{s}_{11} h_{i \alpha} h^{* i \alpha} h_{i \beta}^{\prime} h^{\prime * i \beta}+\tilde{s}_{12} h_{i \alpha} h^{\prime * i \alpha} h_{i \beta}^{\prime} h^{* i \beta}\right] \\
& +\sum_{\alpha, \beta}\left[\tilde{s}_{21}\left(h_{1 \alpha} h^{* 1 \alpha} h_{2 \beta}^{\prime} h^{\prime * 2 \beta}+\text { cycl. }\right)+\tilde{s}_{22}\left(h_{1 \alpha} h^{\prime * 2 \alpha} h_{2 \beta}^{\prime} h^{* 1 \beta}+\text { cycl. }\right)\right] \\
& +i \tilde{s}_{31} \sum_{\alpha, \beta}\left[\left(h_{1 \alpha} h^{\prime * 1 \alpha} h_{2 \beta}^{\prime} h^{* 2 \beta}+\text { cycl. }\right)-\left(h^{* 1 \alpha} h_{1 \alpha}^{\prime} h^{* * 2 \beta} h_{2 \beta}+\text { cycl. }\right)\right] \\
& +i \tilde{s}_{32} \sum_{\alpha, \beta}\left[\left(h_{1 \alpha} h^{* 2 \alpha} h_{2 \beta}^{\prime} h^{* 1 \beta}+\text { cycl. }\right)-\left(h^{* 1 \alpha} h_{2 \alpha} h^{* 2 \beta} h_{1 \beta}^{\prime}+\text { cycl. }\right)\right] .
\end{aligned}
$$

Finally, of the remainder of the potential, only the invariant with coefficient $\tilde{c}$ from Eq. 4.13 needs to be doubled:

$$
\sum_{\alpha, \beta}\left[\tilde{c}_{1}\left(h_{1 \alpha} h^{* 3 \alpha} h_{1 \beta}^{\prime} h^{\prime * 3 \beta}+\text { cycl. }\right)+\tilde{c}_{2}\left(h_{1 \alpha} h^{\prime * 3 \alpha} h_{1 \beta}^{\prime} h^{* 3 \beta}+\text { cycl. }\right)+\text { h.c. }\right] \text {. }
$$

We therefore write

$$
\begin{aligned}
V_{A_{4}}\left(H, H^{\prime}\right) & =V_{0}(H)+V_{0}^{\prime}\left(H^{\prime}\right)+V_{1}\left(H, H^{\prime}\right) \\
& +\sum_{\alpha, \beta}\left[c\left(h_{1 \alpha} h_{1 \beta} h^{* 3 \alpha} h^{* 3 \beta}+\text { cycl. }\right)+c^{\prime}\left(h_{1 \alpha}^{\prime} h_{1 \beta}^{\prime} h^{\prime * 3 \alpha} h^{\prime * 3 \beta}+\text { cycl. }\right)+\text { h.c. }\right] \\
& +\sum_{\alpha, \beta}\left[\tilde{c}_{1}\left(h_{1 \alpha} h^{* 3 \alpha} h_{1 \beta}^{\prime} h^{\prime * 3 \beta}+\text { cycl. }\right)+\tilde{c}_{2}\left(h_{1 \alpha} h^{\prime * 3 \alpha} h_{1 \beta}^{\prime} h^{* 3 \beta}+\text { cycl. }\right)+\text { h.c. }\right] .
\end{aligned}
$$

We note that due to $S U(2)_{L}$ not allowing cubic invariants of $H$ and/or $H^{\prime}$, it is sufficient to use a $Z_{3}$ symmetry to distinguish the $A_{4}$ triplets ${ }^{8}$

This potential generally violates $\mathrm{CP}$. This can be seen from the CP-odd invariants calculated, as $\mathcal{I}_{2}^{(6)}, \mathcal{I}_{3}^{(6)}, \mathcal{I}_{4}^{(6)}, \mathcal{I}_{5}^{(6)}$ (Eqs. 2.50 2.51 2.52 2.53) ) are non-zero (see Table 1 ) but with too large expressions to display here. However, it is possible to impose a CP symmetry with

$$
U_{23}^{H H^{\prime}}=\left(\begin{array}{cc}
U_{23} & 0 \\
0 & U_{23}
\end{array}\right) \otimes \delta_{\beta}^{\alpha}
$$

which, similarly to previous examples, restricts the coefficients in the potential, namely

$$
\tilde{s}_{21}=\tilde{s}_{22}=0 \text {, }
$$

thereby forcing all CPIs to vanish. Imposing, alternatively, the canonical CP symmetry $C P_{0}$ leads to $\tilde{s}_{31}=\tilde{s}_{32}=0$ as well as $c, c^{\prime}, \tilde{c}_{1}, \tilde{c}_{2} \in \mathbb{R}$.

${ }^{8}$ The potential invariant under a $Z_{2} \quad 75$ would additionally allow for invariants of the form $h_{i \alpha} h^{\prime * i \alpha} h_{j \beta} h^{\prime * j \beta}$ and $h_{i \alpha} h^{* i \beta} h_{j \beta} h^{\prime * j \alpha}$ where the conjugated fields are both related to $H^{\prime}$. 


\section{5 $S_{4}$ invariant potentials}

The transition from $\Delta\left(3 n^{2}\right)$ invariant potentials with arbitrary $n \in N$ to potentials which are symmetric under the larger group $\Delta\left(6 n^{2}\right)$ is discussed in Appendix C. The corresponding basis of $S_{4}=\Delta\left(6 \times 2^{2}\right)$ can be found in [12,79,80]. For the $A_{4}$ potential with one triplet of singlets as well the $A_{4}$ potential with a triplet of doublets, the corresponding $S_{4}$ invariant potentials are obtained by setting

$$
c^{*}=c,
$$

so that

$$
V_{S_{4}}(\varphi)=V_{0}(\varphi)+c\left[\left(\varphi_{1} \varphi_{1} \varphi^{* 3} \varphi^{* 3}+\text { cycl. }\right)+\text { h.c. }\right],
$$

and

$$
V_{S_{4}}(H)=V_{0}(H)+\sum_{\alpha, \beta} c\left[\left(h_{1 \alpha} h_{1 \beta} h^{* 3 \alpha} h^{* 3 \beta}+\text { cycl. }\right)+\text { h.c. }\right],
$$

where the potentials $V_{0}$ were defined in Eq. (4.2) and Eq. (4.8). For the potential of two triplets of $A_{4}$, the $S_{4}$ invariant potential arises via setting

$$
\tilde{s}_{2}=\tilde{s}_{3}=0
$$

and additionally

$$
c^{*}=c, \quad c^{*}=c^{\prime}, \quad \tilde{c}^{*}=\tilde{c} .
$$

Defining the following abbreviation,

$$
\begin{aligned}
V_{2}\left(\varphi, \varphi^{\prime}\right)= & \tilde{r}_{1}\left(\sum_{i} \varphi_{i} \varphi^{* i}\right)\left(\sum_{j} \varphi_{j}^{\prime} \varphi^{* j}\right)+\tilde{r}_{2}\left(\sum_{i} \varphi_{i} \varphi^{\prime * i}\right)\left(\sum_{j} \varphi_{j}^{\prime} \varphi^{* j}\right) \\
& +\tilde{s}_{1} \sum_{i}\left(\varphi_{i} \varphi^{* i} \varphi_{i}^{\prime} \varphi^{\prime * i}\right),
\end{aligned}
$$

the full potential of two $S_{4}$ triplets becomes

$$
\begin{aligned}
V_{S_{4}}\left(\varphi, \varphi^{\prime}\right)= & V_{0}(\varphi)+V_{0}^{\prime}\left(\varphi^{\prime}\right)+V_{2}\left(\varphi, \varphi^{\prime}\right)+ \\
& +c\left[\left(\varphi_{1} \varphi_{1} \varphi^{* 3} \varphi^{* 3}+\text { cycl. }\right)+\text { h.c. }\right]+c^{\prime}\left[\left(\varphi_{1}^{\prime} \varphi_{1}^{\prime} \varphi^{\prime * 3} \varphi^{\prime * 3}+\text { cycl. }\right)+\text { h.c. }\right] \\
& +\tilde{c}\left[\left(\varphi_{1} \varphi_{1}^{\prime} \varphi^{* 3} \varphi^{\prime * 3}+\text { cycl. }\right)+\text { h.c. }\right] .
\end{aligned}
$$

The $S_{4}$ potential with two triplets generally conserves CP. This can be understood from the non-vanishing CPIs obtained for $A_{4}$, which were proportional to $\tilde{s}_{2}$ (see Eq. (4.14)) which is zero in the case of $S_{4}$. Indeed, one CP symmetry present in $V_{S_{4}}\left(\varphi, \varphi^{\prime}\right)$ is $U_{23}^{\varphi \varphi^{\prime}}$ in Eq. (4.15), because $S_{4}$ enforces $\tilde{s}_{2}=0$ and therefore the $V_{S_{4}}\left(\varphi, \varphi^{\prime}\right)$ potential is invariant under simultaneous CP transformations with 2-3-swap on $\varphi$ and $\varphi^{\prime}$.

Turning to the case of Higgs doublets of $S U(2)_{L}$, for $V_{A_{4}}\left(H, H^{\prime}\right)$, enlarging the symmetry to $S_{4}$ constrains the potential parameters as follows:

$$
c^{*}=c, \quad c^{*}=c^{\prime}, \quad \tilde{c}_{1}^{*}=\tilde{c}_{1}, \quad \tilde{c}_{2}^{*}=\tilde{c}_{2},
$$


and

$$
\tilde{s}_{21}=\tilde{s}_{22}=\tilde{s}_{31}=\tilde{s}_{32}=0 .
$$

Again, introducing an abbreviation,

$$
\begin{aligned}
V_{2}\left(H, H^{\prime}\right)= & \sum_{i, j, \alpha, \beta}\left[\tilde{r}_{11} h_{i \alpha} h^{* i \alpha} h_{j \beta}^{\prime} h^{* j \beta}+\tilde{r}_{12} h_{i \alpha} h^{* j \alpha} h_{j \beta}^{\prime} h^{* i \beta}\right] \\
& +\sum_{i, j, \alpha, \beta}\left[\tilde{r}_{21} h_{i \alpha} h^{\prime * i \alpha} h_{j \beta}^{\prime} h^{* j \beta}+\tilde{r}_{22} h_{i \alpha} h^{* j \alpha} h_{j \beta}^{\prime} h^{* i \beta}\right] \\
& +\sum_{i, \alpha, \beta}\left[\tilde{s}_{11} h_{i \alpha} h^{* i \alpha} h_{i \beta}^{\prime} h^{\prime * i \beta}+\tilde{s}_{12} h_{i \alpha} h^{* i \alpha} h_{i \beta}^{\prime} h^{* i \beta}\right]
\end{aligned}
$$

the $S_{4}$ invariant potential of two triplets of doublets becomes

$$
\begin{aligned}
V_{S_{4}}\left(H, H^{\prime}\right)= & V_{0}(H)+V_{0}^{\prime}\left(H^{\prime}\right)+V_{2}\left(H, H^{\prime}\right) \\
& +\sum_{\alpha, \beta} c\left[\left(h_{1 \alpha} h_{1 \beta} h^{* 3 \alpha} h^{* 3 \beta}+\text { cycl. }\right)+\text { h.c. }\right] \\
& +\sum_{\alpha, \beta} c^{\prime}\left[\left(h_{1 \alpha}^{\prime} h_{1 \beta}^{\prime} h^{\prime * 3 \alpha} h^{\prime * 3 \beta}+\text { cycl. }\right)+\text { h.c. }\right] \\
& +\sum_{\alpha, \beta} \tilde{c}_{1}\left[\left(h_{1 \alpha} h^{* 3 \alpha} h_{1 \beta}^{\prime} h^{* 3 \beta}+\text { cycl. }\right)+\text { h.c. }\right] \\
& +\sum_{\alpha, \beta} \tilde{c}_{2}\left[\left(h_{1 \alpha} h^{\prime * 3 \alpha} h_{1 \beta}^{\prime} h^{* 3 \beta}+\text { cycl. }\right)+\text { h.c. }\right] .
\end{aligned}
$$

As in Eq. 4.28), the potential $V_{S_{4}}\left(H, H^{\prime}\right)$ conserves CP. As all parameters of this potential are real, it is not surprising, that it is invariant under trivial $\mathrm{CP}, C P_{0}$.

\section{$5 \Delta(27)$ invariant potentials}

In this section we concern ourselves with potentials invariant under $\Delta(27)$. As in the $A_{4}$ case, we consider the field content of a single triplet of SM singlets, then a single triplet which is also an $S U(2)_{L}$ doublet, then two triplets of SM singlets, and finally two $\Delta(27)$ triplets of $S U(2)_{L}$ doublets.

The group $\Delta(27)$ has one irreducible triplet representation $\mathbf{3}$, its conjugate $\overline{\mathbf{3}}$, and nine one-dimensional representations. The product of two triplets decomposes as

$$
\mathbf{3} \otimes \mathbf{3}=(\overline{\mathbf{3}}+\overline{\mathbf{3}})_{s}+\overline{\mathbf{3}}_{a}
$$

where the subscripts $s$ and $a$ denote symmetric and antisymmetric combinations, respectively. In the following we adopt the basis of $12,78,81$.

\subsection{One flavour triplet}

Having only one triplet field, the antisymmetric contribution in Eq. (5.1) vanishes identically. As a consequence there are four independent quartic $\Delta(27)$ invariants of type 
$\mathbf{3} \otimes \mathbf{3} \otimes \overline{\mathbf{3}} \otimes \overline{\mathbf{3}}$. Writing the components of the triplet field as $\varphi_{i}$, with $i=1,2,3$, we can easily derive the renormalisable scalar potential,

$$
V_{\Delta(27)}(\varphi)=V_{0}(\varphi)+\left[d\left(\varphi_{1} \varphi_{1} \varphi^{* 2} \varphi^{* 3}+\text { cycl. }\right)+\text { h.c. }\right] .
$$

The coefficients inside $V_{0}(\varphi)$ (cf. Eq. 4.2) are real but $d \in \mathbb{C}$. The number of independent real parameters is therefore four. $V_{\Delta(27)}(\varphi)$ is accidentally also the potential for a single $\Delta(54)$ triplet [55], as discussed also in Appendix C.

The potential of Eq. (5.2) in its most general form violates $\mathrm{CP}$ as can be seen from the construction of CPIs which do not vanish for general choices of the coefficients in the potential (see Table 1). Calculating the CPIs $\mathcal{I}_{4,5}^{(6)}$ (Eqs. 2.52 2.53) ) explicitly yields the same non-zero result for this potential:

$$
\mathcal{I}_{4,5}^{(6)}=-\frac{3}{32}\left(d^{3}-d^{* 3}\right)\left(d^{3}+6 d d^{*} s+d^{* 3}-8 s^{3}\right)
$$

while the other explicit CPIs that are listed throughout Section 2 are zero for this potential. The potential in Eq. (5.2) is known to be CP conserving in the cases $\operatorname{Arg}(d)=$ $0,2 \pi / 3,4 \pi / 3$. Indeed this is reflected in the CPIs which are proportional to

$$
\left(d^{3}-d^{* 3}\right)
$$

This factor vanishes for $\operatorname{Arg}(d)=0,2 \pi / 3,4 \pi / 3$, where each case corresponds to a distinct $\mathrm{CP}$ symmetry, defined by a $3 \times 3$ matrix $U$. In the following, we explicitly list the $\mathrm{CP}$ transformations that enforce various parameter relations. The $U_{i}$-notation we use in our work matches the indices of the CP transformations listed in [82],

$$
\begin{aligned}
\operatorname{Arg}(d)=0 & \Longleftrightarrow U_{0}=\left(\begin{array}{lll}
1 & 0 & 0 \\
0 & 1 & 0 \\
0 & 0 & 1
\end{array}\right) \text { or } U_{1}=\left(\begin{array}{lll}
1 & 0 & 0 \\
0 & 0 & 1 \\
0 & 1 & 0
\end{array}\right), \\
\operatorname{Arg}(d)=4 \pi / 3 & \Longleftrightarrow U_{2}=\left(\begin{array}{lll}
1 & 0 & 0 \\
0 & 1 & 0 \\
0 & 0 & \omega
\end{array}\right) \text { or } U_{8}=\left(\begin{array}{lll}
\omega & 0 & 0 \\
0 & 0 & 1 \\
0 & 1 & 0
\end{array}\right), \\
\operatorname{Arg}(d)=2 \pi / 3 & \Longleftrightarrow U_{3}=U_{2}^{*}=\left(\begin{array}{lll}
1 & 0 & 0 \\
0 & 1 & 0 \\
0 & 0 & \omega^{2}
\end{array}\right) \text { or } U_{9}=U_{8}^{*}=\left(\begin{array}{ccc}
\omega^{2} & 0 & 0 \\
0 & 0 & 1 \\
0 & 1 & 0
\end{array}\right) .
\end{aligned}
$$

We recall that for each CP transformation an equivalent one can be obtained by multiplying it by an element of $\Delta(27)$. Note also that $U_{1}=U_{23}$ from Eq. (4.5). Focusing on the other factor of Eq. (5.3), all CPIs we have identified vanish if we set

$$
\left(d^{3}+6 d d^{*} s+d^{* 3}-8 s^{3}\right)=0 .
$$

This is a strong hint that there are CP symmetries that make the potential CP conserving, not by fixing the phase of $d$ but by imposing specific relations between the parameters $d$ and $s$. Indeed, there are three solutions to Eq. (5.8) which are listed with the corresponding CP transformations from [82], 


$$
\begin{aligned}
2 s & =\left(d+d^{*}\right)=2 \operatorname{Re}(d) \\
& \Longleftrightarrow U_{4}=\frac{1}{\sqrt{3}}\left(\begin{array}{ccc}
1 & 1 & 1 \\
1 & \omega & \omega^{2} \\
1 & \omega^{2} & \omega
\end{array}\right) \text { or } U_{5}=U_{4} U_{1}=U_{4}^{*}=\frac{1}{\sqrt{3}}\left(\begin{array}{ccc}
1 & 1 & 1 \\
1 & \omega^{2} & \omega \\
1 & \omega & \omega^{2}
\end{array}\right), \\
2 s & =-\operatorname{Re}(d)-\sqrt{3} \operatorname{Im}(d) \\
& \Longleftrightarrow U_{6}=\frac{-i}{\sqrt{3}}\left(\begin{array}{ccc}
1 & \omega & \omega \\
\omega & \omega & 1 \\
\omega & 1 & \omega
\end{array}\right) \text { or } U_{10}=U_{6} U_{1}=\frac{-i}{\sqrt{3}}\left(\begin{array}{ccc}
1 & \omega & \omega \\
\omega & 1 & \omega \\
\omega & \omega & 1
\end{array}\right), \\
2 s & =-\operatorname{Re}(d)+\sqrt{3} \operatorname{Im}(d) \\
& \Longleftrightarrow U_{7}=U_{6}^{*}=\frac{i}{\sqrt{3}}\left(\begin{array}{ccc}
1 & \omega^{2} & \omega^{2} \\
\omega^{2} & \omega^{2} & 1 \\
\omega^{2} & 1 & \omega^{2}
\end{array}\right) \text { or } U_{11}=U_{7} U_{1}=\frac{i}{\sqrt{3}}\left(\begin{array}{ccc}
1 & \omega^{2} & \omega^{2} \\
\omega^{2} & 1 & \omega^{2} \\
\omega^{2} & \omega^{2} & 1
\end{array}\right) .
\end{aligned}
$$

We conclude that there exist $12 \mathrm{CP}$ symmetries, listed in [82], which correspond to two $\mathrm{CP}$ symmetries for each of the $6 \mathrm{CP}$ conserving conditions that make either $\left(d^{3}-\right.$ $\left.d^{* 3}\right)=0$ or $\left(d^{3}+6 d d^{*} s+d^{* 3}-8 s^{3}\right)=0$. The fact that there are two distinct classes of $\mathrm{CP}$ symmetries, unrelated by $\Delta(27)$ transformations, for each of the $6 \mathrm{CP}$ conserving conditions is due to the $\Delta(27)$ potential being accidentally invariant under $\Delta(54)$ [55]. The two classes of $\mathrm{CP}$ symmetries in each case are related to each other by a $\Delta(54)$ transformation.

\subsection{One flavour triplet of Higgs doublets}

If each component of the $\Delta(27)$ triplet is an $S U(2)_{L}$ doublet, the potential is rather similar to the previous case, and in analogy with the $A_{4}$ potential there is one additional invariant which is contained in $V_{0}(H)$. The resulting potential reads

$$
V_{\Delta(27)}(H)=V_{0}(H)+\sum_{\alpha, \beta}\left[d\left(h_{1 \alpha} h_{1 \beta} h^{* 2 \alpha} h^{* 3 \beta}+\text { cycl. }\right)+\text { h.c. }\right] .
$$

In general the potential explicitly violates $\mathrm{CP}$. It is possible to impose $\mathrm{CP}$ conservation as in the previous case, as follows.

Calculating the CPIs, we see that up to a prefactor, $\mathcal{I}_{4,5}^{(6)}$ have the same form as in Eq. (5.3) for the previous potential:

$$
\frac{512}{315} \mathcal{I}_{4}^{(6)}=\frac{1024}{495} \mathcal{I}_{5}^{(6)}=-\left(d^{3}-d^{* 3}\right)\left(d^{3}+6 d d^{*} s+d^{* 3}-8 s^{3}\right) .
$$

This means that the same conditions ensure $\mathrm{CP}$ conservation as in the previous $\Delta(27)$ invariant potential. They are associated to $\mathrm{CP}$ symmetries with the $U_{i}$ matrices discussed 
in the previous subsection, simply multiplied by $\delta_{\beta}^{\alpha}$ acting on $S U(2)_{L}$ indices (similarly to Eq. (4.10).

\subsection{Two flavour triplets}

As for the $A_{4}$ case, we consider the potential involving two physically different flavon fields $\varphi$ and $\varphi^{\prime}$ which both transform in the triplet representation of $\Delta(27)$. Note that the triplet representation of $\Delta(27)$ is unique up to complex conjugation. In addition to the invariants of each field, the full potential contains also mixed terms. Confining ourselves to quartic terms of the form $\varphi \varphi^{\prime} \varphi^{*} \varphi^{*}$ (which can be enforced e.g. by $U(1)$ symmetries, such that the imposed symmetry is really $\left.\Delta(27) \times U(1) \times U(1)^{\prime}\right)$, we obtain nine independent mixed invariants. The resulting renormalisable potential is then given by

$$
\begin{aligned}
V_{\Delta(27)}\left(\varphi, \varphi^{\prime}\right)= & V_{0}(\varphi)+V_{0}^{\prime}\left(\varphi^{\prime}\right)+V_{1}\left(\varphi, \varphi^{\prime}\right) \\
& +\left[d\left(\varphi_{1} \varphi_{1} \varphi^{* 2} \varphi^{* 3}+\text { cycl. }\right)+\text { h.c. }\right]+\left[d^{\prime}\left(\varphi_{1}^{\prime} \varphi_{1}^{\prime} \varphi^{\prime * 2} \varphi^{\prime * 3}+\text { cycl. }\right)+\text { h.c. }\right] \\
& +\left[\tilde{d}_{1}\left(\varphi_{1} \varphi_{1}^{\prime} \varphi^{* 2} \varphi^{\prime * 3}+\text { cycl. }\right)+\text { h.c. }\right]+\left[\tilde{d}_{2}\left(\varphi_{1} \varphi_{1}^{\prime} \varphi^{* 3} \varphi^{\prime * 2}+\text { cycl. }\right)+\text { h.c. }\right] .
\end{aligned}
$$

Here the masses as well as the coupling constants inside $V_{0}, V_{0}^{\prime}$ and $V_{1}$ are all real (note the explicit factor of $i$ multiplying $\tilde{s}_{3}$ ), while the couplings $d, d^{\prime}, \tilde{d}_{1}$ and $\tilde{d}_{2}$ are generally complex.

This potential explicitly violates CP, since several of the CPIs are non-zero as can be seen in Table 1 of Section 7, but the expressions are cumbersome. However it is possible to impose $\mathrm{CP}$ conservation. For example, imposing trivial $\mathrm{CP}\left(C P_{0}\right)$ enforces the 4 complex coefficients $d, d^{\prime}, \tilde{d}_{1}, \tilde{d}_{2}$ to be real and $\tilde{s}_{3}=0$. We have verified explicitly that all CPIs vanish in this case. Alternatively, imposing $U_{23}^{\varphi \varphi^{\prime}}$ Eq. 4.15 enforces $\tilde{s}_{2}=0$ and relates $\tilde{d}_{1}=\tilde{d}_{2}^{*}$ as well as $d^{*}=d, d^{*}=d^{\prime}$, which implies that all the CPIs vanish as expected.

\subsection{Two flavour triplets of Higgs doublets}

As earlier, a potential for two triplets of $S U(2)_{L}$ doublets can be obtained by including all possible $S U(2)_{L}$ contractions of the fields in the $\Delta(27)$ invariants. The only difference of this potential to earlier Higgs potentials lies in the invariants with $d$-coefficients, out of which only the invariants corresponding to $\tilde{d}_{1}$ and $\tilde{d}_{2}$ in Eq. $(5.14)$ need to be doubled. Therefore the potential is in this case:

$$
\begin{aligned}
V_{\Delta(27)}\left(H, H^{\prime}\right) & =V_{0}(H)+V_{0}^{\prime}\left(H^{\prime}\right)+V_{1}\left(H, H^{\prime}\right)+ \\
& +\sum_{\alpha, \beta}\left[d\left(h_{1 \alpha} h_{1 \beta} h^{* 2 \alpha} h^{* 3 \beta}+\text { cycl. }\right)+d^{\prime}\left(h_{1 \alpha}^{\prime} h_{1 \beta}^{\prime} h^{\prime * 2 \alpha} h^{\prime * 3 \beta}+\text { cycl. }\right)+\text { h.c. }\right] \\
& +\sum_{\alpha, \beta}\left[\tilde{d}_{11}\left(h_{1 \alpha} h^{* 2 \alpha} h_{1 \beta}^{\prime} h^{\prime * 3 \beta}+\text { cycl. }\right)+\tilde{d}_{12}\left(h_{1 \alpha} h^{* 3 \alpha} h_{1 \beta}^{\prime} h^{* 2 \beta}+\text { cycl. }\right)+\text { h.c. }\right] \\
& +\sum_{\alpha, \beta}\left[\tilde{d}_{21}\left(h_{1 \alpha} h^{* 3 \alpha} h_{1 \beta}^{\prime} h^{* 2 \beta}+\text { cycl. }\right)+\tilde{d}_{22}\left(h_{1 \alpha} h^{* 2 \alpha} h_{1 \beta}^{\prime} h^{* 3 \beta}+\text { cycl. }\right)+\text { h.c. }\right] .
\end{aligned}
$$


The potential $V_{\Delta(27)}\left(H, H^{\prime}\right)$ is CP violating in general. Of the CPIs calculated, cf. Table 1 . $\mathcal{I}_{2}^{(6)}, \mathcal{I}_{3}^{(6)}, \mathcal{I}_{4}^{(6)}, \mathcal{I}_{5}^{(6)}$ (Eqs. 2.50 2.51 2.52 2.53) are non-zero, but the expressions are too large to display here.

\section{$5.5 \Delta(54)$ invariant potentials}

Working in the basis of [12, 80, 83], the potentials of one triplet of singlets or $S U(2)_{L}$ doublets are both identical for $\Delta(27)$ and $\Delta(54)$. The $\Delta(54)$ symmetric potential of two triplets of $S U(2)_{L}$ singlets is obtained from the corresponding $\Delta(27)$ potential by imposing the constraint of Eq. 4.25),

$$
\tilde{s}_{2}=\tilde{s}_{3}=0
$$

as well as

$$
\tilde{d}_{1}=\tilde{d}_{2}
$$

from which $V_{\Delta(27)}\left(\varphi, \varphi^{\prime}\right)$ becomes

$$
\begin{aligned}
V_{\Delta(54)}\left(\varphi, \varphi^{\prime}\right)= & V_{0}(\varphi)+V_{0}^{\prime}\left(\varphi^{\prime}\right)+V_{2}\left(\varphi, \varphi^{\prime}\right) \\
& +\left[d\left(\varphi_{1} \varphi_{1} \varphi^{* 2} \varphi^{* 3}+\text { cycl. }\right)+\text { h.c. }\right]+\left[d^{\prime}\left(\varphi_{1}^{\prime} \varphi_{1}^{\prime} \varphi^{\prime * 2} \varphi^{\prime * 3}+\text { cycl. }\right)+\text { h.c. }\right] \\
& +\tilde{d}_{1}\left[\left(\varphi_{1} \varphi_{1}^{\prime} \varphi^{* 2} \varphi^{\prime * 3}+\text { cycl. }\right)+\left(\varphi_{1} \varphi_{1}^{\prime} \varphi^{* 3} \varphi^{\prime * 2}+\text { cycl. }\right)\right]+\text { h.c. }
\end{aligned}
$$

The $\Delta(54)$ potential with two triplets does not conserve CP in general, as seen in Table 1. The potential in Eq. (5.18) is obtained from Eq. (5.14) in the $\tilde{s}_{2}=\tilde{s}_{3}=0, \tilde{d}_{1}=\tilde{d}_{2}$ limit, which makes it rather similar to the $\mathrm{CP}$ conserving $V_{\Delta(27)}\left(\varphi, \varphi^{\prime}\right)$ after imposing the $U_{23}^{\varphi \varphi^{\prime}}$ (cf. Eq. 4.15), but note that $V_{\Delta(54)}\left(\varphi, \varphi^{\prime}\right)$ does not have $\tilde{d}_{1}^{*}=\tilde{d}_{1}, d^{*}=d$ nor $d^{\prime *}=d^{\prime}$. Therefore, even though CPI $\mathcal{I}_{2}^{(6)}$ vanishes, $\mathcal{I}_{3}^{(6)}, \mathcal{I}_{4}^{(6)}, \mathcal{I}_{5}^{(6)}$ are non-zero.

For the potential of two triplets of Higgs doublets, the following conditions on the parameters arise when enlarging the symmetry to $\Delta(54)$ :

$$
\tilde{d}_{21}=\tilde{d}_{11}, \quad \tilde{d}_{22}=\tilde{d}_{12}, \quad \tilde{s}_{21}=\tilde{s}_{22}=\tilde{s}_{31}=\tilde{s}_{32}=0 .
$$

The potential becomes

$$
\begin{aligned}
V_{\Delta(54)}\left(H, H^{\prime}\right) & =V_{0}(H)+V_{0}^{\prime}\left(H^{\prime}\right)+V_{2}\left(H, H^{\prime}\right) \\
& +\sum_{\alpha, \beta}\left[d\left(h_{1 \alpha} h_{1 \beta} h^{* 2 \alpha} h^{* 3 \beta}+\text { cycl. }\right)+d^{\prime}\left(h_{1 \alpha}^{\prime} h_{1 \beta}^{\prime} h^{\prime * 2 \alpha} h^{\prime * 3 \beta}+\text { cycl. }\right)+\text { h.c. }\right] \\
& +\sum_{\alpha, \beta}\left[\tilde{d}_{11}\left(h_{1 \alpha} h^{* 2 \alpha} h_{1 \beta}^{\prime} h^{\prime * 3 \beta}+\text { cycl. }\right)+\tilde{d}_{12}\left(h_{1 \alpha} h^{* 3 \alpha} h_{1 \beta}^{\prime} h^{* 2 \beta}+\text { cycl. }\right)+\text { h.c. }\right] \\
& +\sum_{\alpha, \beta}\left[\tilde{d}_{11}\left(h_{1 \alpha} h^{* 3 \alpha} h_{1 \beta}^{\prime} h^{\prime * 2 \beta}+\text { cycl. }\right)+\tilde{d}_{12}\left(h_{1 \alpha} h^{\prime * 2 \alpha} h_{1 \beta}^{\prime} h^{* 3 \beta}+\text { cycl. }\right)+\text { h.c. }\right] .
\end{aligned}
$$

This potential is also generally $\mathrm{CP}$ violating and $\mathcal{I}_{3}^{(6)}, \mathcal{I}_{4}^{(6)}, \mathcal{I}_{5}^{(6)}$ are non-zero but too large to display here. 


\section{$6 \Delta\left(3 n^{2}\right)$ invariant potentials with $n>3$}

So far we have considered the finite groups $A_{4}=\Delta\left(3 \cdot 2^{2}\right)$ and $\Delta(27)=\Delta\left(3 \cdot 3^{2}\right)$ which correspond to the first two non-Abelian members of the series $\Delta\left(3 n^{2}\right)$ with $n \in \mathbb{N}$. In this section we derive renormalisable potentials which are invariant under $\Delta\left(3 n^{2}\right)$ with $n>3$. The field contents considered are a single triplet of SM singlets, then one triplet of $S U(2)_{L}$ doublets, then two triplets of SM singlets and finally two triplets of $S U(2)_{L}$ doublets. Following [78, a triplet of $\Delta\left(3 n^{2}\right)$ can be written as $\mathbf{3}_{(k, l)}$, where $k, l=0,1, \ldots, n-1$. The complex conjugate of $\mathbf{3}_{(k, l)}$ is given by $\mathbf{3}_{(-k,-l)}$, which we sometimes denote as $\overline{\mathbf{3}}$, dropping the indices. The cyclic permutation symmetry included in $\Delta\left(3 n^{2}\right)$ entails an ambiguity in labelling the same triplet representation such that $\mathbf{3}_{(k, l)}=\mathbf{3}_{(l,-k-l)}=\mathbf{3}_{(-k-l, k)}$. With these preliminary remarks, we can determine the product of two identical triplet representations 78

$$
\mathbf{3}_{(k, l)} \otimes \mathbf{3}_{(k, l)}=\left[\mathbf{3}_{(2 k, 2 l)}+\mathbf{3}_{(-k,-l)}\right]_{s}+\left[\mathbf{3}_{(-k,-l)}\right]_{a} .
$$

Again the subscripts $s$ and $a$ denote symmetric and antisymmetric combinations. Assuming the original triplet $\mathbf{3}_{(k, l)}$ to be a faithful (and thus irreducible) representation of $\Delta\left(3 n^{2}\right)$, all representations on the right-hand side are irreducible for $n \neq 2$. Excluding moreover the case with $n=3$, the triplets $\mathbf{3}_{(2 k, 2 l)}$ and $\mathbf{3}_{(-k,-l)}$ denote different representations. Throughout this section we adopt the basis of [12,78.

\subsection{One flavour triplet}

With one triplet field, only the symmetric part of Eq. (6.1) is relevant for constructing quartic terms of the form $\mathbf{3} \otimes \mathbf{3} \otimes \overline{\mathbf{3}} \otimes \overline{\mathbf{3}}$. Considering $n>3$, the two triplets in the symmetric contraction of Eq. 6.1) are distinct, so that only two independent quartic invariants can be constructed. The renormalisable scalar potential, which is additionally invariant under a $U(1)$ symmetry, thus takes the form

$$
V_{\Delta\left(3 n^{2}\right)}(\varphi)=V_{0}(\varphi)
$$

where the explicit form of $V_{0}(\varphi)$ is given in Eq. (4.2). This potential always explicitly conserves CP. It is a reduced version of the $A_{4}$ symmetric potential $V_{A_{4}}(\varphi)$ of Eq. (4.4) which generally conserves CP. Therefore it is clear that $V_{\Delta\left(3 n^{2}\right)}(\varphi)$ is left invariant under the same CP symmetry, i.e. the one defined with a $2-3$ swap, $U_{23}$. In addition, $V_{\Delta\left(3 n^{2}\right)}(\varphi)$ respects the trivial CP symmetry $C P_{0}$ (which $V_{A_{4}}(\varphi)$ in general does not).

\subsection{One flavour triplet of Higgs doublets}

If each component of the faithful $\Delta\left(3 n^{2}\right)$ triplet transforms as an $S U(2)_{L}$ doublet, the corresponding renormalisable potential consists of four independent terms. As described in Section 4, the different ways of contracting the $S U(2)_{L}$ indices entail a doubling of the $\Delta\left(3 n^{2}\right)$ invariant term in Eq. (6.2) which is proportional to $r$. The resulting Higgs potential then takes the form

$$
V_{\Delta\left(3 n^{2}\right)}(H)=V_{0}(H),
$$


with the right-hand side defined in Eq. 4.8). This potential always conserves CP explicitly (for any choice of parameters). Similar to the corresponding $A_{4}$ case, $V_{\Delta\left(3 n^{2}\right)}(H)$ is left invariant under a CP transformation with a 2-3 swap. Additionally, it also respects the trivial CP symmetry $C P_{0}$.

\subsection{Two flavour triplets}

We now turn to the case of two flavour multiplets, $\varphi$ and $\varphi^{\prime}$, in the same faithful triplet representation. The potential can be simplified by imposing individual $U(1)$ symmetries for each of the scalar fields, such that the actual symmetry of the potential is given by $\Delta\left(3 n^{2}\right) \times U(1) \times U(1)^{\prime}$. In addition to the potential of the individual (non-interacting) fields, only mixed terms of the form $\varphi \varphi^{\prime} \varphi^{*} \varphi^{*}$ are possible; in particular cubic terms are absent. In order to construct the mixed quartic terms, we consider the Kronecker product given in Eq. (6.1), now also including the antisymmetric combination. Multiplying the right-hand side with its complex conjugate, we see that there are five independent mixed quartic $\Delta\left(3 n^{2}\right)$ invariants if $n>3$. The renormalisable potential can be written as follows,

$$
V\left(\varphi, \varphi^{\prime}\right)_{\Delta\left(3 n^{2}\right)}=V_{0}(\varphi)+V_{0}^{\prime}\left(\varphi^{\prime}\right)+V_{1}\left(\varphi, \varphi^{\prime}\right)
$$

where the individual contributions to the right-hand side are defined in Eqs. (4.2) and 4.11.

Unlike the previous $\Delta\left(3 n^{2}\right)$ invariant potentials for $n>3$, this potential generally violates $\mathrm{CP}$, as confirmed by the non-zero $\mathrm{CPI} \mathcal{I}_{2}^{(6)}$ (Eq. 2.50$)$ ) which for this potential becomes

$$
\mathcal{I}_{2}^{(6)}=\frac{3}{512} i \tilde{s}_{2} \tilde{s}_{3}\left(-3 \tilde{r}_{2}^{2}+\tilde{s}_{3}^{2}\right)\left(-\tilde{s}_{1}^{2}+\tilde{s}_{1} \tilde{s}_{2}+\tilde{r}_{2}\left(-2 \tilde{s}_{1}+\tilde{s}_{2}\right)+\tilde{s}_{3}^{2}\right) .
$$

Imposing the trivial CP symmetry $C P_{0}$ entails $\tilde{s}_{3}=0$, whereas the $U_{23}^{\varphi \varphi^{\prime}} 2-3$ swap CP symmetry constrains the potential such that $\tilde{s}_{2}=0$. As expected, both CP symmetries enforce $\mathcal{I}_{2}^{(6)}=0$ (and make any other CPIs vanish), but they are distinct CP symmetries with distinct effects on the potential.

Inspection of other CPIs reveals that also the factor $\left(-3 \tilde{r}_{2}^{2}+\tilde{s}_{3}^{2}\right)$ is present in each nonvanishing CPI we found. This raises the question if there exists a CP symmetry which is associated with setting this factor to zero. Such a symmetry must relate different terms of the potential in Eq. (6.4), namely

$$
\tilde{r}_{2}\left(\sum_{i} \varphi_{i} \varphi^{\prime * i}\right)\left(\sum_{j} \varphi_{j}^{\prime} \varphi^{* j}\right)+i \tilde{s}_{3}\left[\left(\varphi_{1} \varphi^{* 1} \varphi_{2}^{\prime} \varphi^{* 2}+\text { cycl. }\right)-\left(\varphi^{* 1} \varphi_{1}^{\prime} \varphi^{* 2} \varphi_{2}+\text { cycl. }\right)\right]
$$

Clearly, the term proportional to $\tilde{r}_{2}$ is invariant under a general CP transformation where the unitary matrix $U$ is block diagonal and the blocks are the same for both triplets $\varphi$ and $\varphi^{\prime}$. Hence, we are led to more general choices with different $3 \times 3$ blocks $U_{\varphi}$ and $U_{\varphi^{\prime}}$ for $\varphi$ and $\varphi^{\prime}$, respectively. Pursuing the simple ansatz

$$
U^{\varphi \varphi^{\prime}}=\left(\begin{array}{cc}
U_{\varphi} & 0 \\
0 & U_{\varphi^{\prime}}
\end{array}\right), \quad \text { with } \quad U_{\varphi}=\left(\begin{array}{ccc}
1 & 0 & 0 \\
0 & \omega & 0 \\
0 & 0 & \omega^{2}
\end{array}\right), \quad U_{\varphi^{\prime}}=\left(\begin{array}{ccc}
1 & 0 & 0 \\
0 & \omega^{2} & 0 \\
0 & 0 & \omega
\end{array}\right)
$$


we find that the potential remains invariant under the corresponding general $\mathrm{CP}$ transformation if and only if

$$
\tilde{s}_{3}=\tilde{r}_{2} i\left(\omega-\omega^{2}\right) .
$$

Inserting $\omega=e^{2 \pi i / 3}$, we get $\tilde{s}_{3}=-\sqrt{3} \tilde{r}_{2}$ which corresponds to one solution of the quadratic equation $3 \tilde{r}_{2}^{2}-\tilde{s}_{3}^{2}=0$. The other solution, $\tilde{s}_{3}=\sqrt{3} \tilde{r}_{2}$, is related to the CP transformation where the roles of the explicit matrices in Eq. (6.6) are exchanged. Imposing either of the two CP symmetries guarantees that all CPIs vanish.

An example of a larger non-trivial CPI is provided by $\mathcal{I}_{1}^{(7,2)}$, defined in Eq. (B.137) of Appendix B.6. Explicit evaluation in the parametrisation of Eq. (6.4) yields

$$
\begin{aligned}
\mathcal{I}_{1}^{(7,2)}= & \frac{9}{8192} i \tilde{s}_{2} \tilde{s}_{3}\left(3 \tilde{r}_{2}^{2}-\tilde{s}_{3}^{2}\right)\left(m_{\varphi}^{2}-m_{\varphi^{\prime}}^{2}\right)^{2}\left(\tilde{r}_{1}+\tilde{r}_{2}+\tilde{s}_{1}\right) \times\left[16\left(s^{2}+s s^{\prime}+s^{\prime 2}\right)+\right. \\
& \left.+8 r\left(2 s+s^{\prime}\right)+8 r^{\prime}\left(2 s^{\prime}+s\right)+\tilde{s}_{1}^{2}+\tilde{s}_{2}^{2}-\tilde{s}_{3}^{2}-\tilde{s}_{1} \tilde{s}_{2}+\tilde{r}_{2}\left(2 \tilde{s}_{1}-\tilde{s}_{2}\right)\right] .
\end{aligned}
$$

While this more complicated CPI vanishes for $m_{\varphi}=m_{\varphi^{\prime}}$, we already know that such a relation is not a consequence of any CP symmetry because the simpler CPI derived above does not depend on the masses. In other words, any CP symmetry that would relate the masses by $m_{\varphi}=m_{\varphi^{\prime}}$ would have to impose additional constraints on the other parameters of the potential.

Having identified the CP symmetries corresponding to the zeros of $\tilde{s}_{2} \tilde{s}_{3}\left(3 \tilde{r}_{2}^{2}-\tilde{s}_{3}^{2}\right)$, one may wonder about the consequences of imposing other CP symmetries on the potential of Eq. (6.4). As an example, one could for instance consider the situation where $U$ is given by the block matrix where $U_{\varphi}$ and $U_{\varphi}^{\prime}$ are both given by one of the matrices of Eq. (5.9). A straightforward but somewhat tedious calculation reveals that such a "general" CP symmetry would require vanishing coefficients for all non-SU(3) type terms. In other words $s=s^{\prime}=\tilde{s}_{1}=\tilde{s}_{2}=\tilde{s}_{3}=0$. The symmetry of the resulting potential would therefore be enhanced from $\Delta\left(3 n^{2}\right)$ to $S U(3)$ in addition to preserving CP.

\subsection{Two flavour triplets of Higgs doublets}

The potential of two triplets of $S U(2)_{L}$ doublets can be deduced from the potential of two flavour triplets of $S U(2)_{L}$ singlets. It is a particular case of the corresponding $A_{4}$ potential. We therefore write the potential in terms of the expressions defined in Eqs. (4.8) and 4.17,

$$
V_{\Delta\left(3 n^{2}\right)}\left(H, H^{\prime}\right)=V_{0}(H)+V_{0}^{\prime}\left(H^{\prime}\right)+V_{1}\left(H, H^{\prime}\right)
$$

We note again that due to the $S U(2)_{L} \times U(1)_{Y}$ gauge group, the potential cannot contain any cubic terms. In fact, each term must have an equal number of Higgs and complex conjugate Higgs fields. Hence it is sufficient to impose e.g. a $Z_{3}$ symmetry with nontrivial charge for only one of the two triplets of Higgs doublets in order to enforce the potential of Eq. (6.9). This potential in Eq. 6.9) generally violates CP explicitly. Of the CP-odd invariants calculated, cf. Table $1 . \mathcal{I}_{2}^{(6)}, \mathcal{I}_{3}^{(6)}, \mathcal{I}_{4}^{(6)}, \mathcal{I}_{5}^{(6)}$ (Eqs. 2.50 2.51 2.52 2.53) are non-zero, but the expressions are too large to display here. 


\section{5 $\Delta\left(6 n^{2}\right)$ invariant potentials with $n>3$}

Working in the basis of [12,80], it is straightforward to enhance the symmetry of $\Delta\left(3 n^{2}\right)$ invariant potentials to $\Delta\left(6 n^{2}\right)$ by imposing extra constraints, see Appendix C. With only one flavour triplet $\varphi$ or $H$, the renormalisable potentials are automatically symmetric under $\Delta\left(6 n^{2}\right)$, i.e.

$$
V_{\Delta\left(6 n^{2}\right)}(\varphi)=V_{\Delta\left(3 n^{2}\right)}(\varphi)=V_{0}(\varphi), \quad V_{\Delta\left(6 n^{2}\right)}(H)=V_{\Delta\left(3 n^{2}\right)}(H)=V_{0}(H),
$$

where $V_{0}(\varphi)$ and $V_{0}(H)$ are defined in Eqs. (4.2) and (4.8), respectively. With two flavour triplets, it is necessary to impose $\tilde{s}_{2}=\tilde{s}_{3}=0$ for $V_{\Delta\left(6 n^{2}\right)}\left(\varphi, \varphi^{\prime}\right)$ and $\tilde{s}_{21}=\tilde{s}_{22}=\tilde{s}_{31}=$ $\tilde{s}_{32}=0$ for $V_{\Delta\left(6 n^{2}\right)}\left(H, H^{\prime}\right)$. Using the definitions of Eqs. 4.27) and 4.31), we then have

$$
\begin{aligned}
V_{\Delta\left(6 n^{2}\right)}\left(\varphi, \varphi^{\prime}\right) & =V_{0}(\varphi)+V_{0}^{\prime}\left(\varphi^{\prime}\right)+V_{2}\left(\varphi, \varphi^{\prime}\right), \\
V_{\Delta\left(6 n^{2}\right)}\left(H, H^{\prime}\right) & =V_{0}(H)+V_{0}^{\prime}\left(H^{\prime}\right)+V_{2}\left(H, H^{\prime}\right) .
\end{aligned}
$$

All of the above $\Delta\left(6 n^{2}\right)$ invariant potentials (with $n>3$ ) conserve $\mathrm{CP}$ explicitly. For instance, one can easily show that the respective trivial $\mathrm{CP}$ transformations $C P_{0}$ as well as the respective $\mathrm{CP}$ transformations with a 2-3 swap $\left(U_{23}, U_{23}^{H}, U_{23}^{\varphi \varphi^{\prime}}, U_{23}^{H H^{\prime}}\right)$ do not constrain the parameters of the potentials as they are all real.

\section{Summary of CPIs for explicit CP violation}

In this section, we collect our results of Sections 4, 5 and 6. We have calculated CPIs for a number of different potentials which are invariant under either of the following discrete symmetries $A_{4}, S_{4}, \Delta(27), \Delta(54), \Delta\left(3 n^{2}\right)$ and $\Delta\left(6 n^{2}\right)$ with $n>3$. All these symmetries have irreducible triplet representations. Choosing Higgs fields in a faithful triplet, we have determined the potential for one triplet of $S U(2)_{L}$ singlets, one triplet of $S U(2)_{L}$ doublets, two triplets of $S U(2)_{L}$ singlets and finally two triplets of $S U(2)_{L}$ doublets. The (scalar) particle content for each of these $6 \times 4$ cases is listed intuitively in the leftmost column of Table 1 .

Many of the CPIs defined in Section 2 vanish for all of these 24 potentials. We have checked explicitly that $\mathcal{I}_{1}^{(2,2)}, \mathcal{I}_{1}^{(3,1)}, \mathcal{I}_{2}^{(3,1)}, \mathcal{I}_{1}^{(3,2)}, \mathcal{I}_{2}^{(3,2)}, \mathcal{I}_{1}^{(4,1)}, \mathcal{I}_{1}^{(5)}, \mathcal{I}_{2}^{(5)}, \mathcal{I}_{3}^{(5)}, \mathcal{I}_{1}^{(6)}$ vanish in all cases. Table 1 shows the relevant invariants $\mathcal{I}_{2}^{(6)}, \mathcal{I}_{3}^{(6)}, \mathcal{I}_{4}^{(6)}, \mathcal{I}_{5}^{(6)}$, evaluated for each potential. A 0-entry means that the corresponding CPI was found to be zero. A non-vanishing CPI is indicated by either an asterisk or an equation number, where the latter refers to the position in our paper where the corresponding expression for the CPI is given. The asterisk is used for non-zero CPIs which we have calculated analytically but whose expressions are too large to display in the text.

We observe from Table 1 that 12 potentials feature explicit $\mathrm{CP}$ violation. On the other hand, all four CPIs shown in the table vanish for the other 12 potentials, which suggests $\mathrm{CP}$ is conserved in those cases. Indeed, as listed in the rightmost column, one can easily identify CP transformations which leave the potential unchanged, thereby explicitly proving that $\mathrm{CP}$ is conserved. We recall that trivial $\mathrm{CP}\left(C P_{0}\right)$ means complex conjugation on all scalar fields, cf. Eq. 2.21). "NA" stands for "Not Applicable" and is used for CP violating cases. 


\begin{tabular}{|c|c|c|c|c|c|}
\hline & $\mathcal{I}_{2}^{(6)}$ & $\mathcal{I}_{3}^{(6)}$ & $\mathcal{I}_{4}^{(6)}$ & $\mathcal{I}_{5}^{(6)}$ & $\mathrm{CP}$ \\
\hline$\left(\mathbf{3}_{A_{4}}, \mathbf{1}_{S U(2)_{L}}\right)$ & 0 & 0 & 0 & 0 & Eq. (4.5) \\
\hline$\left(\mathbf{3}_{A_{4}}, \mathbf{2}_{S U(2)_{L}}\right)$ & 0 & 0 & 0 & 0 & Eq. $(4.10)$ \\
\hline $2 \times\left(\mathbf{3}_{A_{4}}, \mathbf{1}_{S U(2)_{L}}\right)$ & * & * & * & * & $\mathrm{NA}$ \\
\hline $2 \times\left(\mathbf{3}_{A_{4}}, \mathbf{2}_{S U(2)_{L}}\right)$ & $*$ & $*$ & $*$ & * & NA \\
\hline$\left(\mathbf{3}_{\Delta(27)}, \mathbf{1}_{S U(2)_{L}}\right)$ & 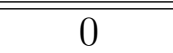 & 0 & Eq. (5.3) & Eq. (5.3) & NA \\
\hline$\left(\mathbf{3}_{\Delta(27)}, \mathbf{2}_{S U(2)_{L}}\right)$ & 0 & 0 & Eq. $(\sqrt{5.13})$ & Eq. $(\sqrt{5.13})$ & NA \\
\hline $2 \times\left(\mathbf{3}_{\Delta(27)}, \mathbf{1}_{S U(2)_{L}}\right)$ & * & * & & & NA \\
\hline $2 \times\left(\mathbf{3}_{\Delta(27)}, \mathbf{2}_{\left.S U(2)_{L}\right)}\right.$ & * & $*$ & * & * & $\mathrm{NA}$ \\
\hline$\left(\mathbf{3}_{\Delta\left(3 n^{2}\right)}, \mathbf{1}_{S U(2)_{L}}\right)$ & $\overline{0}$ & 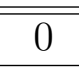 & 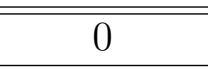 & 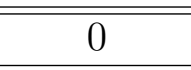 & Eq. 4.5 \\
\hline$\left(\mathbf{3}_{\Delta\left(3 n^{2}\right)}, \mathbf{2}_{S U(2)_{L}}\right)$ & 0 & 0 & 0 & 0 & Eq. 4.10$)$ \\
\hline $2 \times\left(\mathbf{3}_{\Delta\left(3 n^{2}\right)}, \mathbf{1}_{S U(2)_{L}}\right)$ & Eq. $(6.5)$ & * & * & * & NA \\
\hline $2 \times\left(\mathbf{3}_{\Delta\left(3 n^{2}\right)}, \mathbf{2}_{S U(2)_{L}}\right)$ & $*$ & $*$ & $*$ & $*$ & $\mathrm{NA}$ \\
\hline$\overline{\left(\mathbf{3}_{S_{4}}, \mathbf{1}_{S U(2)_{L}}\right)}$ & $\overline{0}$ & 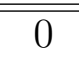 & $\overline{\overline{0}}$ & $\overline{0}$ & $C P_{0} \&$ Eq. 4.5 \\
\hline$\left(\mathbf{3}_{S_{4}}, \mathbf{2}_{S U(2)_{L}}\right)$ & 0 & 0 & 0 & 0 & $C P_{0} \&$ Eq. 4.10 \\
\hline $2 \times\left(\mathbf{3}_{S_{4}}, \mathbf{1}_{S U(2)_{L}}\right)$ & 0 & 0 & 0 & 0 & $C P_{0} \&$ Eq. $(\overline{4.15})$ \\
\hline $2 \times\left(\mathbf{3}_{S_{4}}, \mathbf{2}_{S U(2)_{L}}\right)$ & 0 & 0 & 0 & 0 & $C P_{0} \&$ Eq. $(4.20$ \\
\hline$\left(\mathbf{3}_{\Delta(54)}, \mathbf{1}_{S U(2)_{L}}\right)$ & 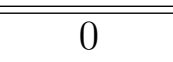 & 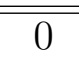 & * & * & $\overline{\mathrm{NA}}$ \\
\hline$\left(\mathbf{3}_{\Delta(54)}, \mathbf{2}_{S U(2)_{L}}\right)$ & 0 & 0 & * & * & NA \\
\hline $2 \times\left(\mathbf{3}_{\Delta(54)}, \mathbf{1}_{S U(2)_{L}}\right)$ & 0 & * & * & * & $\mathrm{NA}$ \\
\hline $2 \times\left(\mathbf{3}_{\Delta(54)}, \mathbf{2}_{S U(2)_{L}}\right)$ & 0 & * & * & * & NA \\
\hline$\left(\mathbf{3}_{\Delta\left(6 n^{2}\right)}, \mathbf{1}_{S U(2)_{L}}\right)$ & 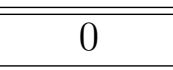 & $\overline{0}$ & 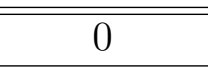 & 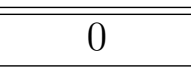 & $C P_{0} \&$ Eq. 4.5 \\
\hline$\left(\mathbf{3}_{\Delta\left(6 n^{2}\right)}, \mathbf{2}_{S U(2)_{L}}\right)$ & 0 & 0 & 0 & 0 & $C P_{0} \&$ Eq. $(\overline{4.10}$ \\
\hline $2 \times\left(\mathbf{3}_{\triangle\left(6 n^{2}\right)}, \mathbf{1}_{S U(2)_{L}}\right)$ & 0 & 0 & 0 & 0 & $C P_{0} \&$ Eq. (4.15) \\
\hline $2 \times\left(\mathbf{3}_{\Delta\left(6 n^{2}\right)}, \mathbf{2}_{S U(2)_{L}}\right)$ & 0 & 0 & 0 & 0 & $C P_{0} \&$ Eq. \\
\hline
\end{tabular}

Table 1: Summary of CPIs and (if applicable) CP symmetry transformations for scalar potentials with discrete symmetry.

\section{CP-odd invariants for spontaneous CP violation}

So far we have discussed CPIs that signal explicit CP violation in scalar potentials. It is also useful to consider CPIs that indicate the presence of spontaneous CP violation. In order to extend our formalism (which is applicable to any potentials once translated into the standard form) we need to include also VEVs.

Recall that VEVs transform as vectors under basis transformations, cf. Eqs. (2.22) and (2.23):

$$
\begin{aligned}
v_{a} & \mapsto V_{a}^{a^{\prime}} v_{a^{\prime}}, \\
v^{* a} & \mapsto v^{* a^{\prime}} V_{a^{\prime}}^{\dagger a} .
\end{aligned}
$$

When used in invariants, first, if the potential does not contain trilinear couplings, VEVs can only appear in pairs of $v$ and corresponding $v^{*}$ because otherwise indices would remain uncontracted. Furthermore, all VEVs commute and thus can be combined into one large 
tensor,

$$
W_{w_{1}^{\prime} \ldots w_{n_{v}}^{\prime}}^{w_{1} \ldots w_{n_{v}}}=v_{w_{1}^{\prime}} \ldots v_{w_{n_{v}}^{\prime}} v^{* w_{1}} \ldots v^{* w_{n_{v}}}
$$

where $n_{v}$ is the number of $v, v^{*}$ pairs $9^{9}$ Using $W$, all invariants with $n_{v}$ pairs of VEV and conjugated VEV can be written using

$$
\begin{aligned}
J_{\sigma}^{\left(n_{v}, m_{Y}, n_{Z}\right)} & \equiv W_{\sigma\left(w_{1}\right) \ldots \sigma\left(w_{n_{v}}\right)}^{w_{1} \ldots w_{n_{v}}} Y_{\sigma\left(a_{1}\right)}^{a_{1}} \ldots Y_{\sigma\left(a_{m_{Y}}\right)}^{a_{m_{Y}}} Z_{\sigma\left(b_{1}\right) \sigma\left(b_{2}\right)}^{b_{1} b_{2}} \ldots Z_{\sigma\left(b_{2 n_{Z}-1}\right) \sigma\left(b_{2 n_{Z}}\right)}^{b_{2 n_{Z}-1 b_{2 n_{Z}}}} \\
& \mapsto W_{w_{1} \ldots w_{n_{v}}}^{\sigma\left(w_{1}\right) \ldots \sigma\left(w_{n_{v}}\right)} Y_{a_{1}}^{\sigma\left(a_{1}\right)} \ldots Y_{a_{m_{Y}}^{\sigma\left(a_{m_{Y}}\right)}}^{\sigma\left(b_{1}\right) \sigma\left(b_{2}\right)} \ldots Z_{b_{1} b_{2}}^{\sigma\left(b_{2 n_{Z}-1}\right) \sigma\left(b_{2 n_{Z}}\right)} \equiv\left(J_{\sigma}^{\left(n_{v}, m_{Y}, n_{Z}\right)}\right)^{*}
\end{aligned}
$$

with $\sigma \in S_{n_{v}+m_{Y}+2 n_{Z}}$. When drawing diagrams, there are additional rules for contractions with VEVs, again with $X=Y, Z$ :

$$
X_{. .}^{a . .} v_{a}=\bullet \longrightarrow \times
$$

and

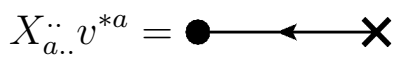

Invariants containing only $Y$ tensors and VEVs are always CP-even. The smallest examples of CPIs for spontaneous CP violation built from $Z$ tensors and VEVs are

$$
J_{1}^{(3,1)} \equiv Z_{a_{5} a_{6}}^{a_{1} a_{2}} Z_{a_{1} a_{3}}^{a_{3} a_{4}} Z_{a_{2} a_{7}}^{a_{5} a_{6}} v_{a_{4}} v^{* a_{1} a_{3}} Z_{a_{5} a_{6}}^{a_{3} a_{4}} v_{a_{2}} v_{a_{4}} v^{* a_{5}} v^{* a_{6}}
$$

where the superscripts on $J$ indicate the number of $Z$ tensors and pairs of VEVs in the invariant. A complete search for invariants with $\left(n_{Z}, n_{v}\right)=(2,2),(3,1),(3,2),(4,1)$ was performed. The method is explained in Appendix $\mathrm{A}$ and the invariants not given in the main text are listed in Appendix B.4.

${ }^{9} \mathrm{In}$ [26, VEVs are always assigned in pairs to matrices $V_{b}^{a}=v^{* a} v_{b}$, however, since all VEVs commute, even for four or more VEVs, also all $V_{b}^{a}$ commute and can be summarised in one large totally symmetric tensor. 


\subsection{Minimisation condition in terms of diagrams}

The minima of the a potential written as in Eq. (2.1) fulfil

$$
0=\frac{\partial V}{\partial \phi_{e}}=\phi^{* a} Y_{a}^{e}+2 \phi^{* a} \phi^{* c} Z_{a c}^{e d} \phi_{d}
$$

and

$$
0=\frac{\partial V}{\partial \phi^{* e}}=Y_{e}^{b} \phi_{b}+2 \phi^{* c} Z_{e c}^{b d} \phi_{b} \phi_{d},
$$

where the factor of 2 appears because of the symmetry of $Z_{a c}^{b d}$ under $b \leftrightarrow d$ and $a \leftrightarrow c$. Replacing the fields by their VEVs, these minimisation conditions can be expressed in terms of diagrams:

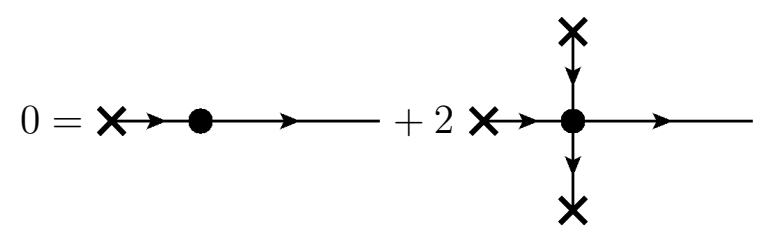

and

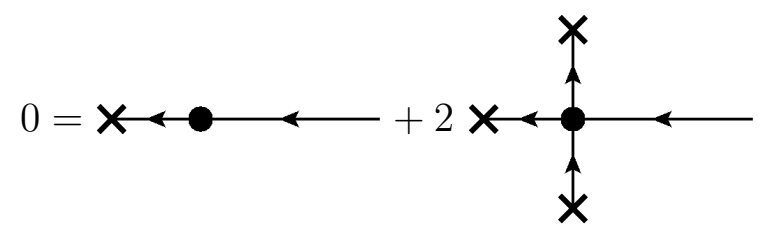

This can be used later to simplify CPIs, as can be seen by applying

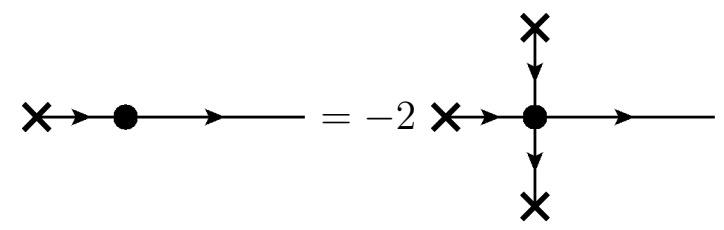

in Eq. 8.7). Using the minimisation condition Eq. 8.14 , the invariant $J_{1}^{(2,2)}$ can be simplified to 10

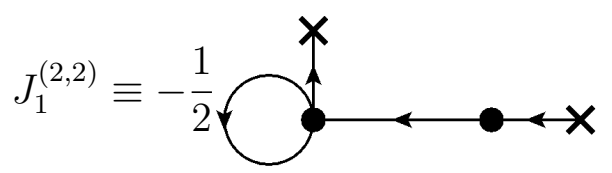

This can only be CP-odd if $Y$ is not proportional to the identity. One can now search for more complicated invariants built from $Z$ tensors and VEVs that will not simplify like this. The smallest CPIs for spontaneous $\mathrm{CP}$ violation without self-loops which also cannot be simplified using the minimisation condition for $n_{Z}=3,4$ respectively are

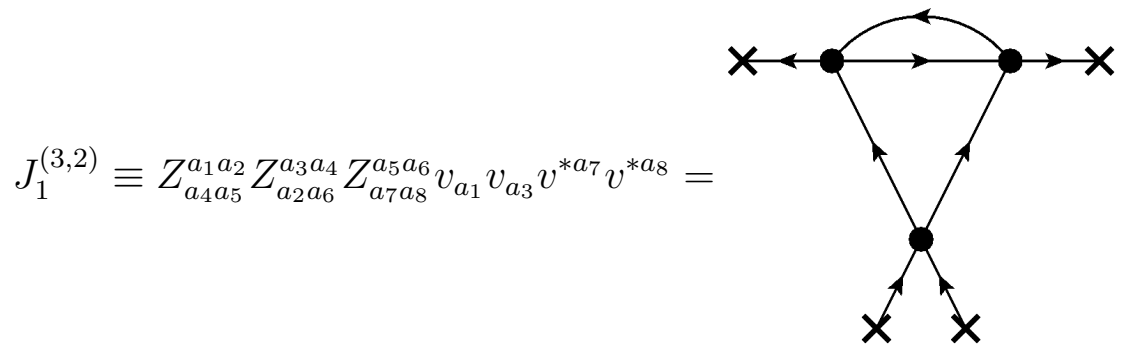

\footnotetext{
${ }^{10}$ The resulting expression corresponds to the invariant $J_{3}$ in Eq. (26) of 24 .
} 
and

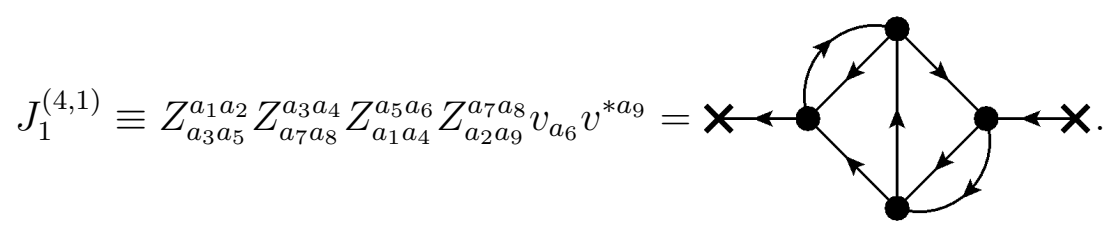

\subsection{Example applications}

\subsubsection{One triplet of $\boldsymbol{A}_{4}$}

As we have seen, the potential in Eq. 4.9 conserves CP explicitly. By an analysis of all VEVs, it has been shown [64 that CP cannot be spontaneously broken. Using our approach we have verified that the low order invariants vanish. In particular, all spontaneous invariants up to $n_{Z}=3, n_{v}=2$ are found to vanish for this potential.

\subsubsection{One triplet of $\Delta(27)$}

One can now calculate SCPIs for this potential for arbitrary VEVs and the smallest nonzero SCPI found is $\mathcal{J}_{1}^{(3,2)}$, as defined via Eq. 8.16$)$. For the general potential $V_{\Delta(27)}(\varphi)$ (which we note is $\mathrm{CP}$ violating), it takes the value

$$
\begin{aligned}
\mathcal{J}_{1}^{(3,2)}= & \frac{1}{4}\left(d^{* 3}-d^{3}\right)\left(\left|v_{1}\right|^{4}+\left|v_{2}\right|^{4}+\left|v_{3}\right|^{4}-2\left|v_{1}\right|^{2}\left|v_{2}\right|^{2}-2\left|v_{1}\right|^{2}\left|v_{3}\right|^{2}-2\left|v_{2}\right|^{2}\left|v_{3}\right|^{2}\right) \\
& +\frac{1}{2}\left(d d^{* 2}-2 d^{*} s^{2}+d^{2} s\right)\left(v_{2} v_{3} v_{1}^{* 2}+v_{1} v_{3} v_{2}^{* 2}+v_{1} v_{2} v_{3}^{* 2}\right) \\
& -\frac{1}{2}\left(d^{2} d^{*}-2 d s^{2}+d^{* 2} s\right)\left(v_{2}^{*} v_{3}^{*} v_{1}^{2}+v_{1}^{*} v_{3}^{*} v_{2}^{2}+v_{1}^{*} v_{2}^{*} v_{3}^{2}\right)
\end{aligned}
$$

In order to demonstrate the usefulness of SCPIs, let us consider the following special cases of $V_{\Delta(27)}(\varphi)$ where we impose different CP symmetries. We start by considering trivial $\mathrm{CP}\left(C P_{0}\right)$, which in this case is the $U_{0}$ matrix, forcing $\operatorname{Arg}(d)=0$ which simplifies the SCPI expression to

$\mathcal{J}_{1}^{(3,2)}=\frac{1}{2}\left(d^{3}-2 d s^{2}+d^{2} s\right)\left[\left(v_{2} v_{3} v_{1}^{* 2}+v_{1} v_{3} v_{2}^{* 2}+v_{1} v_{2} v_{3}^{* 2}\right)-\left(v_{2}^{*} v_{3}^{*} v_{1}^{2}+v_{1}^{*} v_{3}^{*} v_{2}^{2}+v_{1}^{*} v_{2}^{*} v_{3}^{2}\right)\right]$

It is known 52,55 57] that the complex $\operatorname{VEV}\left(1, \omega, \omega^{2}\right)$ is not $\mathrm{CP}$ violating when starting with trivial CP. This can be confirmed easily by using the SCPI above. Instead, the geometrically $\mathrm{CP}$ violating $\operatorname{VEV}(\omega, 1,1)$ does give non-zero when plugged into the SCPI. Let us consider now the CP symmetry $U_{3}$, forcing $\operatorname{Arg}(d)=2 \pi / 3$. Because $d$ remains complex, even a real VEV like $(1,1,1)$ spontaneously violates $\mathrm{CP}$ 66 and this is shown by the SCPI:

$$
\mathcal{J}_{1}^{(3,2)}=\frac{1}{2} \operatorname{Im}\left(d d^{* 2}-2 d^{*} s^{2}+d^{2} s\right)\left[\left(3 v_{1}^{4}\right)\right] .
$$

Another interesting case is the CP symmetry $U_{4}$, forcing $2 s=\left(d+d^{*}\right)=2 \operatorname{Re}(d)$. This simplifies Eq. (8.18) to

$$
\mathcal{J}_{1}^{(3,2)}=\frac{1}{4}\left(d^{* 3}-d^{3}\right)\left(\left|v_{1}\right|^{4}+\left|v_{2}\right|^{4}+\left|v_{3}\right|^{4}-2\left|v_{1}\right|^{2}\left|v_{2}\right|^{2}-2\left|v_{1}\right|^{2}\left|v_{3}\right|^{2}-2\left|v_{2}\right|^{2}\left|v_{3}\right|^{2}\right) .
$$


It is interesting that in this case the SCPI indicates that spontaneous $\mathrm{CP}$ violation is independent of the phases of the VEV. Indeed, the known VEVs for the $U_{0}$ symmetric potential, such as $(0,0,1),(1,1,1)$ (which are real) and $(\omega, 1,1)$ are still candidate VEVs of the $U_{4}$ symmetric potential and all violate CP spontaneously, as indicated by the SCPI.

\section{Conclusions}

In this paper we have been concerned with CPV arising from scalar potentials which go beyond the one Higgs doublet of the SM. After reviewing the well-known general technique of constructing CPIs, we have introduced and developed powerful tools based on diagrams and contraction matrices that allow to systematically identify CPIs in an efficient and straightforward way. Such CPIs, which are valid for any scalar potential, provide a reliable indicator for whether $\mathrm{CP}$ is explicitly violated by the parameters of the potential. We have also extended our formalism to construct the spontaneous CPIs involving the VEVs, in order to reliably determine whether $\mathrm{CP}$ is spontaneously violated.

In order to illustrate the usefulness of the CPI approach, we then applied our results to multi-Higgs scalar potentials of physical interest. We first considered the general 2HDM case which was known to be CP violating, with a complete basis of CPIs known, with several small CPIs being non-zero. We then considered 3HDM and 6HDM which are symmetric under $\Delta\left(3 n^{2}\right)$ and $\Delta\left(6 n^{2}\right)$ groups. Many of these potentials had not been studied before and the new CPIs we found with our systematic search were needed as the previously known ones vanish even for potentials where the new CPIs reveal the presence of explicit CP violation.

For each potential, we either determined the lowest order non-zero CPIs (thereby proving that potential is $\mathrm{CP}$ violating) or, in cases where all the considered CPIs vanish, we derived the explicit CP symmetries that leave the potential invariant (thereby proving that potential is CP conserving). Since the potentials considered were very symmetric, we found that most of the smaller CPIs vanish. Although the CPIs apply to any potential, they take different expressions as functions of the parameters of the potential, as clearly illustrated in the 2HDM example. Furthermore, CPIs that are useful for one potential can vanish for other $\mathrm{CP}$ violating potentials.

We found that the $A_{4}$ potentials, although generally $\mathrm{CP}$ conserving for one triplet of Higgs doublets or singlets, are no longer CP conserving in general when two $A_{4}$ triplets are present (either doublets or singlets). By contrast we find that $\Delta(27)$ potentials are all $\mathrm{CP}$ violating in general. Although the $\Delta(27)$ potentials with a single triplet (whether the scalars are Higgs doublets or not) had previously been studied extensively, by using the calculated expression for a CPI we completely mapped specific CP symmetries to different ways to make the CPI expression vanish. For such potentials, we further analysed spontaneous $\mathrm{CP}$ violation when considering different $\mathrm{CP}$ symmetries by using a non-trivial SCPI. The potentials with $\Delta\left(3 n^{2}\right)$ with $n>3$ turn out to be particular cases of $A_{4}$ potentials. For such cases it is notable that the expressions for the non-zero CPI become manageable for the case with two triplets (non-Higgs), which allowed us to find a CP symmetry that relates two of the real parameters of the potential. Moreover, we found that all of the $\Delta\left(6 n^{2}\right)$ potentials are special cases of the respective $\Delta\left(3 n^{2}\right)$ potentials. In 
the $S_{4}$ case, this makes even the potentials with two triplets automatically CP conserving. Although the $\Delta(54)$ potential for one triplet (whether the scalars are Higgs doublets or not) coincides with the $\Delta(27)$ potential, this is no longer the case when two triplets are present, but they still generally violate CP. $\Delta\left(6 n^{2}\right)$ with $n>3$ is a particular case of $S_{4}$ and therefore the potentials considered are again automatically $\mathrm{CP}$ conserving.

Finally, we briefly showed how our approach may also be applied to spontaneous CPV. As an illustration of this we calculated the SCPIs which are relevant for a $\Delta(27)$ potential showing how it reveals the CP properties of candidate VEVs.

In conclusion, the invariant approach to $\mathrm{CP}$ violation provides a reliable method for studying the $\mathrm{CP}$ properties of multi-Higgs potentials. We have developed a systematic formalism for determing the CPIs for multi-Higgs potentials in general, and have extensively applied this formalism to both the familiar general 2HDM as well as many examples in which the Higgs fields fall into irreducible triplet representations of a discrete symmetry. We considered not only SM Higgs doublets, but also SM singlets which play the role of flavons in flavour models. In each case we catalogued all the lowest order CPIs, many of which previously unknown, thereby elucidating the CP properties of the considered potentials and finding the relevant $\mathrm{CP}$ symmetry transformations where applicable.

\section{Acknowledgements}

This project has received funding from the European Union's Seventh Framework Programme for research, technological development and demonstration under grant agreement no PIEF-GA-2012-327195 SIFT. SFK and TN acknowledge partial support from the STFC Consolidated ST/J000396/1 grant and the European Union FP7 ITN-INVISIBLES (Marie Curie Actions, PITN-GA-2011-289442). The work of CL is supported by the Deutsche Forschungsgemeinschaft (DFG) within the Research Unit FOR 1873 "Quark Flavour Physics and Effective Field Theories". The authors thank Celso Nishi for bringing to their attention an error in Section 3 of the previous version of the manuscript.

\section{Appendix}

\section{A Symmetries of invariants}

In the main text, invariants both without and with VEVs were defined via permutations of indices, cf. Eqs. 2.37) and (8.4). Firstly, it might seem as if there is a huge number of invariants, one for each possible permutation of indices, whose number grows as the factorial of the number of indices. But luckily, as already hinted at in subsection 2.2. invariants have symmetries which will reduce the number of inequivalent invariants. Secondly, one still has to find those index permutations which correspond to CPIs. This appendix concerns itself with these two issues.

Invariants were defined in the following way via index permutations $\sigma \in S_{n}$ where $n$ is the total number of upper indices coming from all involved tensors and VEVs: for 
invariants without VEVs,

$$
I_{\sigma}^{\left(m_{Y} n_{Z}\right)}=Y_{\sigma\left(a_{1}\right)}^{a_{1}} \ldots Y_{\sigma\left(a_{m_{Y}}\right)}^{a_{m_{Y}}} Z_{\sigma\left(b_{1}\right) \sigma\left(b_{2}\right)}^{b_{1} b_{2}} \ldots Z_{\sigma\left(b_{2 n_{Z}-1}\right) \sigma\left(b_{2 n_{Z}}\right)}^{b_{2 n_{Z}} b_{2 n_{Z}}} \text { with } \sigma \in S_{m_{Y}+2 n_{Z}}
$$

where $n=m_{Y}+2 n_{Z}$, and for invariants containing VEVs,

$$
\begin{aligned}
& J_{\sigma}^{\left(n_{v}, m_{Y}, n_{Z}\right)}=W_{\sigma\left(w_{1}\right) \ldots \sigma\left(w_{n_{v}}\right)}^{w_{1} \ldots w_{n_{v}}} Y_{\sigma\left(a_{1}\right)}^{a_{1}} \ldots Y_{\sigma\left(a_{m_{Y}}\right)}^{a_{m_{Y}}} Z_{\sigma\left(b_{1}\right) \sigma\left(b_{2}\right)}^{b_{1} b_{2}} \ldots Z_{\sigma\left(b_{2 n_{Z}-1}\right) \sigma\left(b_{2 n_{Z}}\right)}^{b_{2 n_{Z}{ }^{-1} b_{2 n_{Z}}}} \\
& \mapsto W_{w_{1} \ldots w_{n_{v}}}^{\sigma\left(w_{1}\right) \ldots \sigma\left(w_{n_{v}}\right)} Y_{a_{1}}^{\sigma\left(a_{1}\right)} \ldots Y_{a_{m_{Y}}}^{\sigma\left(a_{m_{Y}}\right)} Z_{b_{1} b_{2}}^{\sigma\left(b_{1}\right) \sigma\left(b_{2}\right)} \ldots Z_{b_{2 n_{Z}-1} b_{2 n_{Z}}}^{\sigma\left(b_{2 n_{Z}}\right) \sigma\left(b_{2 n_{Z}}\right)}=\left(J_{\sigma}^{\left(n_{v}, m_{Y}, n_{Z}\right)}\right)^{*}
\end{aligned}
$$

where now $n=n_{v}+m_{Y}+2 n_{Z}$ and $W$ as defined in Eq. (8.3).

There are the following sources of symmetries of invariants: renaming of indices, permutations of tensors of the same type, and internal symmetries of tensors. Internal symmetries of tensors can refer to symmetries under exchanging indices on the tensor, and symmetries induced by the symmetry of the Lagrangian. Except for the latter, which are not discussed here, all of these symmetries exist for arbitrary invariants corresponding to arbitrary potentials. These sources of symmetries will now be discussed. To streamline notation, write all indices into a multi-index,

$$
\alpha=\left(a_{1}, \ldots, a_{n}\right)
$$

where now permutations act on $\alpha$ by acting on each index as usual:

$$
\sigma(\alpha)=\left(\sigma\left(a_{1}\right), \ldots, \sigma\left(a_{n}\right)\right)
$$

Also, let $\mathcal{Z}$ stand for the product of tensors (both $Y$ and $Z$ ) and VEVs appropriate to the invariant in discussion, then any invariant can be written as

$$
I_{\sigma}=\mathcal{Z}_{\sigma(\alpha)}^{\alpha}
$$

Renaming indices into each other corresponds to another permutation of all indices. For $a_{i} \mapsto \pi\left(a_{i}\right)$ with $\pi \in S_{n}$, the invariant becomes

$$
I_{\sigma} \stackrel{\pi}{\rightarrow} \mathcal{Z}_{\sigma(\pi(\alpha))}^{\pi(\alpha)}
$$

The original invariant and the invariant with indices renamed into each other have the same value.

Next, some elements of invariants are symmetric under independent permutations of upper and lower indices. For example, as discussed in section 2.2, the following four versions of the $Z$ tensor are equal,

$$
Z_{c d}^{a b}=Z_{c d}^{b a}=Z_{d c}^{a b}=Z_{d c}^{b a}
$$

because $Z_{c d}^{a b}$ is symmetric under $a \leftrightarrow b$ and/or $c \leftrightarrow d$. This means that for each $Z$ tensor in the invariant there are 4 equivalent ways of connecting it to the rest of the invariant and thus for $n_{Z} Z$ tensors, there would be $4^{n_{Z}} \sigma$ matrices producing the same invariant and diagram. Similarly, in the tensor $W$ that summaries the product of all VEVs and complex 
conjugates of VEVs, all upper and lower indices can be permuted independently of each other. Denoting any such permutation of indices that is allowed by internal symmetries of tensors by $\tau$, then this condition becomes

$$
\mathcal{Z}_{\sigma(\alpha)}^{\alpha}=\mathcal{Z}_{\sigma(\alpha)}^{\tau(\alpha)}=\mathcal{Z}_{\tau(\sigma(\alpha))}^{\alpha}=\mathcal{Z}_{\tau(\sigma(\alpha))}^{\tau(\alpha)}=I_{\sigma}
$$

These internal symmetries can be taken into account in the actual search for CPIs by defining a new matrix that is produced from one of the equivalent $\sigma$ matrices, which maps all invariants that are related by transformations of the type $\tau$ onto a single matrix that also uniquely corresponds to the diagram corresponding to all these invariants. (In the diagram the symmetries are taken into account automatically.) This new matrix will be called contraction matrix and denoted by $m$. Define the following submatrices of $\sigma$ and $m$ :

$$
\sigma=\left(\begin{array}{ccc}
\sigma_{v v} & \sigma_{v Y} & \sigma_{v Z} \\
\sigma_{Y v} & \sigma_{Y Y} & \sigma_{Y Z} \\
\sigma_{Z v} & \sigma_{Z Y} & \sigma_{Z Z}
\end{array}\right), m=\left(\begin{array}{ccc}
m_{v v} & m_{v Y} & m_{v Z} \\
m_{Y v} & m_{Y Y} & m_{Y Z} \\
m_{Z v} & m_{Z Y} & m_{Z Z}
\end{array}\right)
$$

where now the $v v$ parts correspond to contractions between VEVs, $v Y$ between VEVs and $Y$ tensors, and so on, until $Z Z$, which corresponds to contractions between $Z$ tensors. While $\sigma$ is a $n \times n$ matrix with $n$ the number of indices, $m$ will be a $N \times N$ matrix where $N$ is the number of tensors in the invariant. $W$ would only be counted once. The relations between the submatrices of $\sigma$ and $m$ are as follows:

$$
\begin{aligned}
m_{v v} & =\sum_{i, j}\left(\sigma_{v v}\right)_{i j} \\
\left(m_{v Y}\right)_{j} & =\sum_{i}\left(\sigma_{v Y}\right)_{i j} \\
\left(m_{v Z}\right)_{j} & =\sum_{i}\left(\sigma_{v Z}\right)_{2 i-1, j}+\sum_{i}\left(\sigma_{v Z}\right)_{2 i, j}, \\
\left(m_{Y v}\right)_{i} & =\sum_{j}\left(\sigma_{Y v}\right)_{i j}, \\
\left(m_{Z v}\right)_{i} & =\sum_{j}\left(\sigma_{Z v}\right)_{i, 2 j-1}+\sum_{j}\left(\sigma_{Z v}\right)_{i, 2 j}, \\
\left(m_{Y Y}\right)_{i j} & =\sigma_{i j} \\
\left(m_{Y Z}\right)_{i j} & =\sigma_{i, 2 j-1}+\sigma_{i, 2 j}, \\
\left(m_{Z Y}\right)_{i j} & =\sigma_{2 i-1, j}+\sigma_{2 i, j}, \\
\left(m_{Z Z}\right)_{i j} & =\sigma_{2 i-1,2 j-1}+\sigma_{2 i-1,2 j}+\sigma_{2 i, 2 j-1}+\sigma_{2 i, 2 j} .
\end{aligned}
$$

The element $m_{i j}$ denotes how many arrows are pointing from the $i$-th tensor in the invariant to the $j$-th tensor. What is happening in Eq. A.10 is that all equivalent ways of contracting the $i$-th and $j$-th tensor are summarised in $m_{i j}$ which means that e.g. for a contraction from a $Y$ tensor to a $Z$ tensor, one has to add the two elements corresponding to the two possible permutations of the lower index of $Z$, out of which only one can be non-zero in $\sigma$. Similarly, for contractions of a $Z$ tensor with another $Z$ tensor (or itself), one has to add all entries in the $2 \times 2$ submatrix that corresponds to the four involved indices, out of which only two can be non-zero in $\sigma$. 
For an invariant that only consists of $Y$ tensors, the contraction matrix $m$ is identical to $\sigma$. For invariants only consisting of $Z$ tensors, the situation also becomes a little simpler, as the full contraction matrix is given by the last line of Eq. (A.10). As $\sigma$ is a permutation matrix, in a $2 \times 2$ submatrix only either the two diagonal or the two off-diagonal elements can be non-zero at the same time and the contraction matrix decays into the sum of two smaller permutation matrices of only $n_{Z}$ elements, i.e. with $\sigma_{1}^{Z}, \sigma_{2}^{Z} \in S_{n_{Z}}$ :

$$
m_{i j}=\left(\sigma_{1}^{Z}\right)_{i j}+\left(\sigma_{2}^{Z}\right)_{i j}
$$

Now, one can discuss the last source of symmetry, namely permutations of tensors of the same type. Interchanging the position of two $Z$ tensors in an invariant,

$$
Z_{\sigma\left(a_{1}\right) \sigma\left(a_{2}\right)}^{a_{1} a_{2}} Z_{\sigma\left(a_{3}\right) \sigma\left(a_{4}\right)}^{a_{3} a_{4}} \cdots \rightarrow Z_{\sigma\left(a_{3}\right) \sigma\left(a_{4}\right)}^{a_{3} a_{4}} Z_{\sigma\left(a_{1}\right) \sigma\left(a_{2}\right)}^{a_{1} a_{2}} \cdots,
$$

induces simultaneous permutations of both upper and lower indices of the form

$$
\tilde{\tau}=\left(\begin{array}{cccc}
0 & 1 & & \\
1 & 0 & & \\
& & 1 & \\
& & & \ddots
\end{array}\right) \otimes\left(\begin{array}{ll}
1 & 0 \\
0 & 1
\end{array}\right),
$$

such that an invariant transforms as

$$
I_{\sigma} \stackrel{\tilde{\tau}}{\rightarrow} \mathcal{Z}_{\tilde{\tau}(\sigma(\alpha))}^{\tilde{\tau}(\alpha)}
$$

For larger invariants that also contain $Y$ tensors and VEVs the index transformation induced by permutations of tensors of equal type works similarly. Now one can rename $\tilde{\tau}(\alpha) \equiv \alpha^{\prime}$ such that the invariant becomes

$$
I_{\sigma} \rightarrow \mathcal{Z}_{\tilde{\tau}\left(\sigma\left(\tilde{\tau}^{-1}\left(\alpha^{\prime}\right)\right)\right)}^{\alpha^{\prime}},
$$

which shows that permutations of tensors relate different $\sigma$ matrices in a way similar to conjugacy class transformations, except that the index permutations induced by tensor permutations do not generate the full permutation group $S_{n}$ of the $n$ indices. To summarise the symmetries of $\sigma$, all permutation matrices that are related to $\sigma$ by conjugation with transformations of type $\tau$, Eq. A.8) and transformations of type $\tilde{\tau}$, Eq. A.15,

$$
\tilde{\tau} \circ \tau \circ \sigma \circ \tau^{\prime} \circ \tilde{\tau}^{-1}
$$

where $\tau$ and $\tau^{\prime}$ can be two different transformations, produce the same invariant as $\sigma$.

On a contraction matrix $m$, the permutations on tensors act in a simpler way. For all $\sigma^{Y} \in S_{m_{Y}}$ and $\sigma^{Z} \in S_{n_{Z}}$, all of the contraction matrices, first for invariants without $\mathrm{VEVs}$,

$$
\left(\begin{array}{cc}
\sigma_{Y} & 0 \\
0 & \sigma_{Z}
\end{array}\right) m\left(\begin{array}{cc}
\sigma_{Y} & 0 \\
0 & \sigma_{Z}
\end{array}\right)^{T}
$$

and for invariants with VEVs,

$$
\left(\begin{array}{ccc}
1 & 0 & 0 \\
0 & \sigma_{Y} & 0 \\
0 & 0 & \sigma_{Z}
\end{array}\right) m\left(\begin{array}{ccc}
1 & 0 & 0 \\
0 & \sigma_{Y} & 0 \\
0 & 0 & \sigma_{Z}
\end{array}\right)^{T}
$$


will produce equivalent invariants. Similarly, for invariants only involving $Z$ tensors, all $\sigma^{Z} m\left(\sigma^{Z}\right)^{T}$ will produce equivalent invariants. Applying this to Eq. (A.11), this means that one of the two summands can be chosen to be a conjugacy class representative of $S_{n_{Z}}$ which reduces the number of invariants that need to be considered.

Finally, all pieces are in place to discuss the CP properties first of $\sigma$ and after that of $m$. The CP conjugate of an invariant can be obtained by interchanging upper and lower indices, or in the shorthand notation introduced in Eq. (A.5),

$$
I_{\sigma}=\mathcal{Z}_{\sigma(\alpha)}^{\alpha} \stackrel{C P}{\longrightarrow} \mathcal{Z}_{\alpha}^{\sigma(\alpha)}
$$

One can now rename $\sigma(\alpha)=\alpha^{\prime}$ and subsequently drop the prime to obtain

$$
I_{\sigma} \stackrel{C P}{\longrightarrow} \mathcal{Z}_{\sigma^{-1}(\alpha)}^{\alpha}
$$

Naively, an invariant is CP-even if it equals its CP conjugate which leads to the condition

$$
\sigma^{2}=1
$$

However, one has to take into account also all permutation matrices that are equivalent to $\sigma$ such that the condition becomes

$$
\sigma^{-1}=\tilde{\tau} \circ \tau \circ \sigma \circ \tau^{\prime} \circ \tilde{\tau}^{-1}
$$

which means that as soon as any $\tau, \tau^{\prime}, \tilde{\tau}$ exist such that the above condition can be fulfilled, $\sigma$ produces a $\mathrm{CP}$-even invariant.

For contraction matrices, the condition testing if an invariant is CP-even simplifies. With $\sigma^{-1}=\sigma^{T}$, from which follows that $m \stackrel{C P}{\longrightarrow} m^{T}$ and if $\sigma_{Y}$ and $\sigma_{Z}$ exist, such that the right-hand side is fulfilled, then the condition for invariants without VEVs to be CP-even becomes

$$
\text { Invariant CP-even } \Leftrightarrow m^{T}=\left(\begin{array}{cc}
\sigma_{Y} & 0 \\
0 & \sigma_{Z}
\end{array}\right) m\left(\begin{array}{cc}
\sigma_{Y} & 0 \\
0 & \sigma_{Z}
\end{array}\right)^{T}
$$

and for invariants with VEVs

$$
\text { spont. Invariant CP-even } \Leftrightarrow m^{T}=\left(\begin{array}{ccc}
1 & 0 & 0 \\
0 & \sigma_{Y} & 0 \\
0 & 0 & \sigma_{Z}
\end{array}\right) m\left(\begin{array}{ccc}
1 & 0 & 0 \\
0 & \sigma_{Y} & 0 \\
0 & 0 & \sigma_{Z}
\end{array}\right)^{T}
$$

where the actions of $\tau$ and $\tau^{\prime}$ are absorbed in $m$. Figures 2 and 3 contain examples of contraction matrices for small diagrams. There, all contraction matrices happen to be trivially symmetric except for the CPIs.

It is condition Eq. A.24 that was used to find CP-odd invariants. In the actual search, first all $\sigma$ matrices for a certain number of $Y$ and $Z$ tensors, and VEVs was generated. This list of $\sigma$ matrices was then reduced to a list of contraction matrices, which was condensed using Eq. (A.18) to classes of equivalent contraction matrices, out of which a representative was tested for CP-oddness using Eq. A.24). This search was performed for invariants without VEVs for $m_{Y}=0$ up to $n_{Z}=6$, where it was found that all invariants without $Y$ tensors until $n_{Z}=4$ are CP even. Furthermore, CP-odd 


$$
\begin{aligned}
& Y_{a}^{a}=\bigcirc_{=(1)} \\
& Y_{a}^{a} Y_{b}^{b}=\bigcirc=\left(\begin{array}{ll}
1 & 0 \\
0 & 1
\end{array}\right) \\
& Y_{b}^{a} Y_{a}^{b}=\left(\begin{array}{ll}
0 & 1 \\
1 & 0
\end{array}\right) \\
& Z_{a b}^{a b}=\bigcirc=(2) \\
& Z_{b c}^{a c} Y_{a}^{b}=\bigcirc=\left(\begin{array}{ll}
0 & 1 \\
1 & 1
\end{array}\right) \\
& Z_{c d}^{a b} Z_{a b}^{c d}= \\
& Z_{a c}^{a b} Z_{b d}^{c d}=\bigcirc=\left(\begin{array}{ll}
1 & 1 \\
1 & 1
\end{array}\right) \\
& I_{1} \equiv Z_{a e}^{a b} Z_{b f}^{c d} Y_{c}^{e} Y_{d}^{f}=\longrightarrow\left(\begin{array}{llll}
0 & 0 & 1 & 0 \\
0 & 0 & 0 & 1 \\
0 & 0 & 1 & 1 \\
1 & 1 & 0 & 0
\end{array}\right)
\end{aligned}
$$

Figure 2: Examples of contraction matrices for small invariants. All contraction matrices are symmetric except for the CPI. 


$$
J_{1}^{(3,1)}=Z_{a_{5} a_{6}}^{a_{1} a_{2}} Z_{a_{1} a_{3}}^{a_{3} a_{4}} Z_{a_{2} a_{7}}^{a_{5} a_{6}} v_{a_{4}} v^{* a_{7}}=\left(\begin{array}{llll}
0 & 0 & 0 & 1 \\
0 & 0 & 1 & 1 \\
1 & 0 & 1 & 0 \\
0 & 2 & 0 & 0
\end{array}\right)
$$

Figure 3: Examples for contraction matrices of CPIs for spontaneous CP violation. We draw each of the VEVs (as opposed to a single vertex for the whole $W$ tensor).

invariants were found for $\left(m_{Y}, n_{Z}\right)=(1,3),(1,4),(2,2),(2,3),(3,3)$. For invariants with VEVs, only a search for invariants with $m_{Y}=0$ was performed, where CP-odd invariants were found for $\left(n_{v}, n_{Z}\right)=(1,3),(2,3),(1,4)$. All inequivalent invariants from these classes are listed in section 2 or in appendix $B$.

As one progresses to more complicated invariants, one has to make sure not to count invariants that are products or powers of smaller invariants. An invariant that is a product of two smaller invariants will correspond to a diagram that decays into two separate graphs. As this means that some vertices are only connected among each other while being unconnected to the rest of the diagram, such a reducible invariant will be described by a contraction matrix that can be brought to block-diagonal form only using permutation matrices. This means in particular, as for invariants with VEVs, $m_{v v}$ denotes the number of VEVs that are only connected to other VEVs, that $m_{v v} \neq 0$ would mean that the diagram would contain graphs for $v_{a} v^{* a}$ that are unconnected to the rest of the diagram.

Finally, there is one last condition that relates invariants, namely the minimisation condition Eq. 8.12). In the contraction matrix for an invariant with VEVs this can be used if there is an $i$ such that

$$
m_{1 i}=1 \text { and } m_{i 1}=2
$$

or

$$
m_{1 i}=2 \text { and } m_{i 1}=1 .
$$

In both cases, the $\mathrm{Z}$ tensor at position $i$ in the invariant is connected to three VEVs. 


\section{B List of invariants}

B.1 Contraction matrices of $n_{Z}=5$ invariants
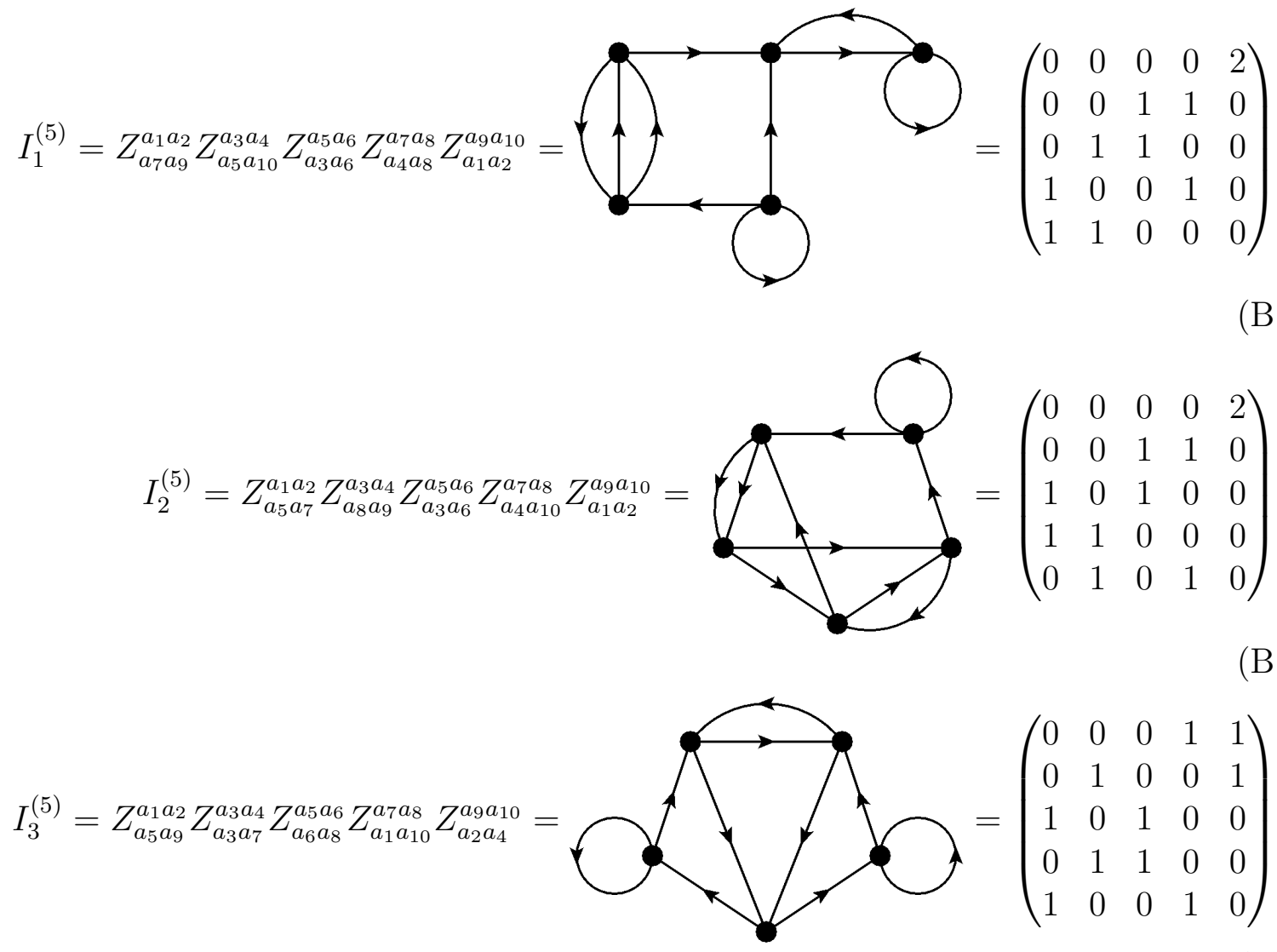

B.2 Contraction matrices of $n_{Z}=6$ invariants without $Z$-selfloops

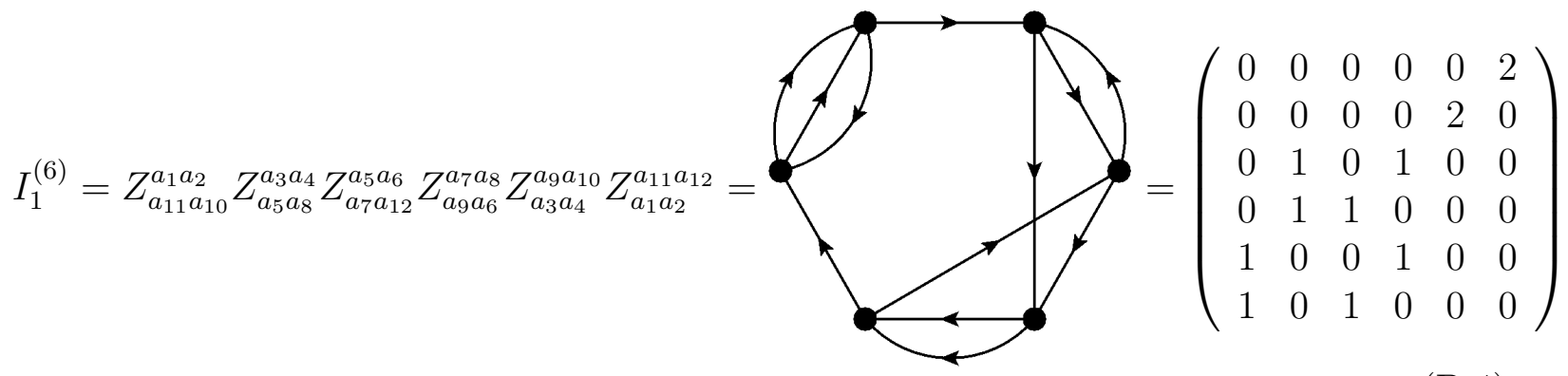



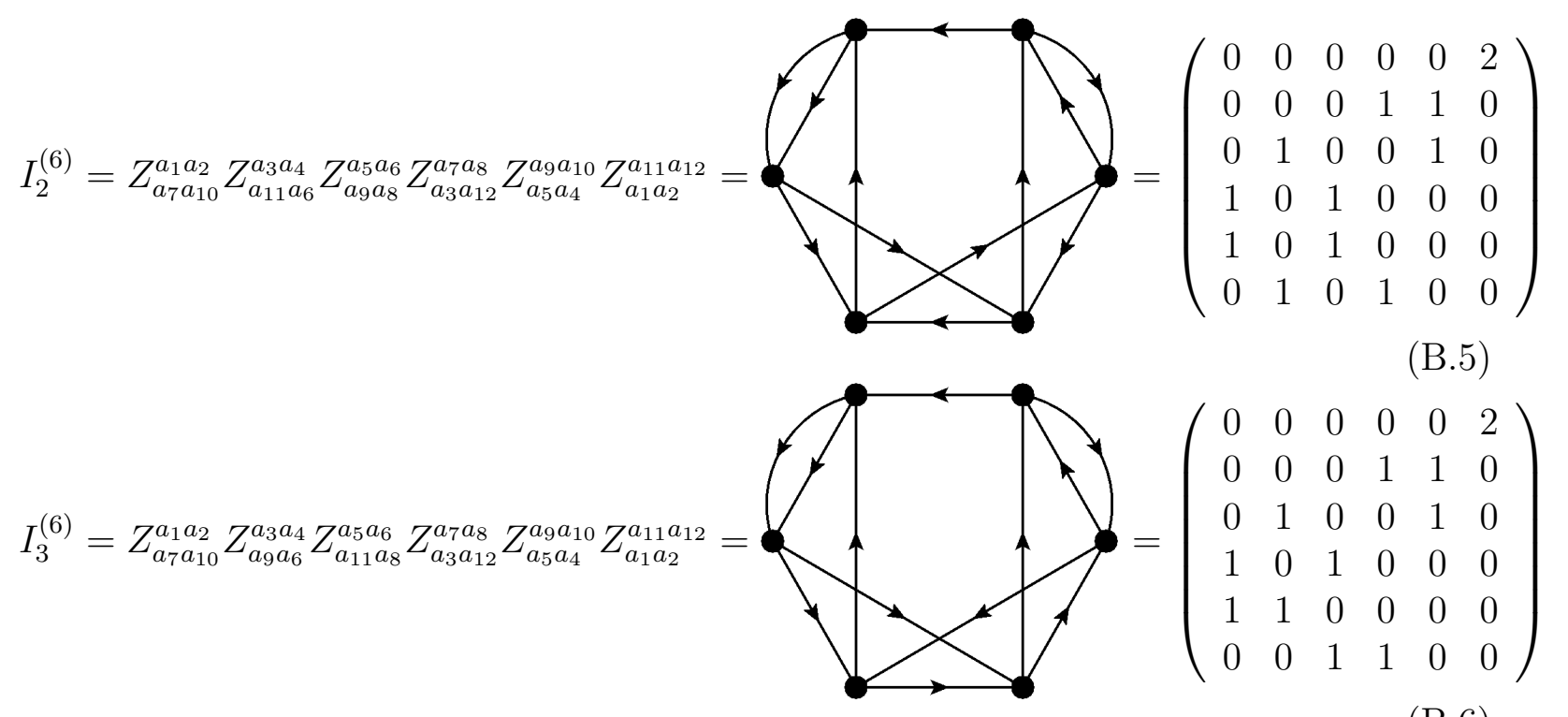

(B.6)
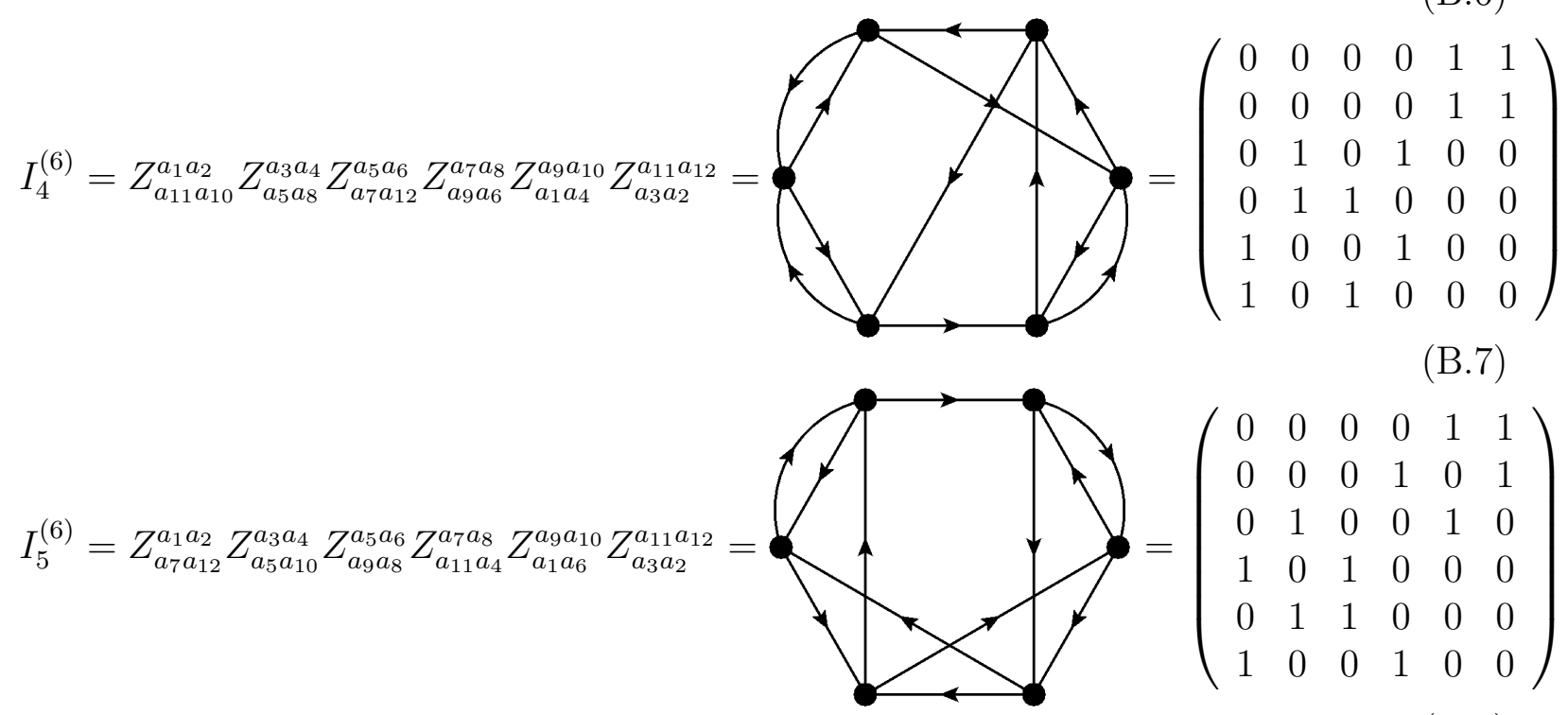


\section{B.3 $n_{Z}=6$ invariants with self-loops}

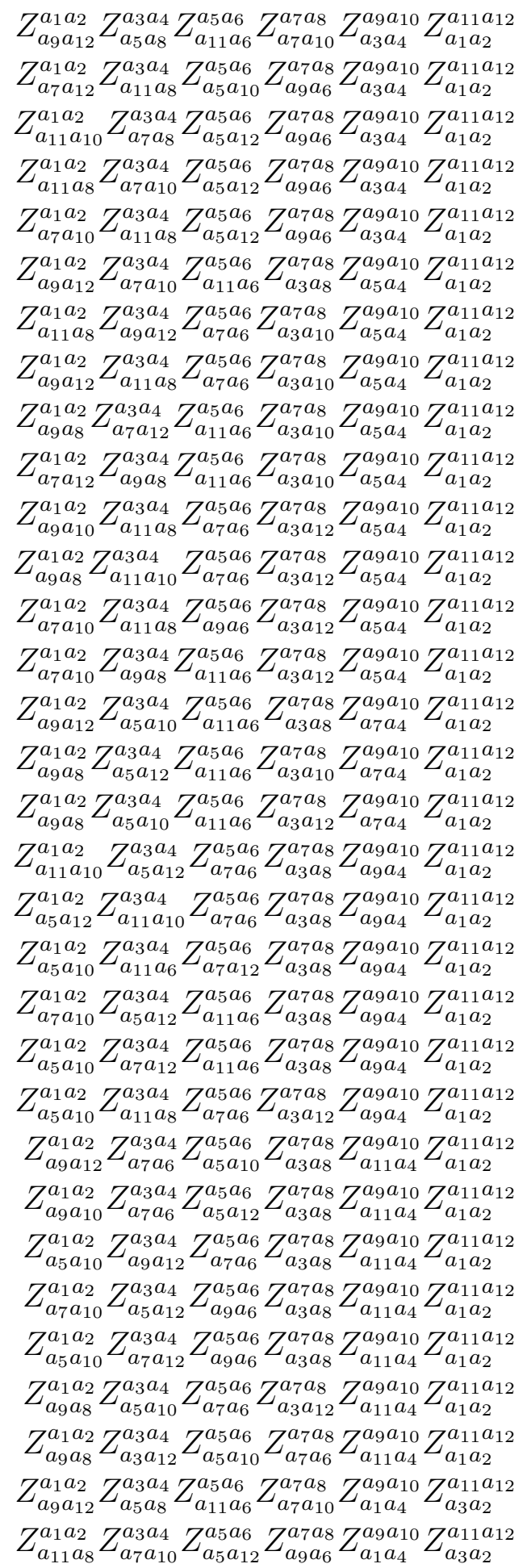




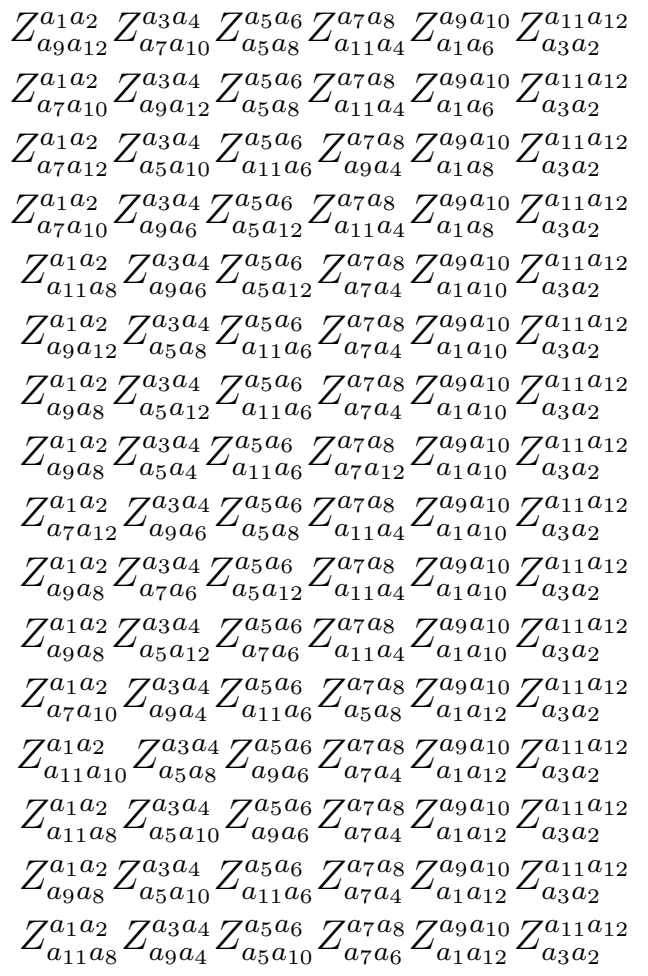

\section{B.4 Invariants with $n_{Y} \neq 0$ not listed in the main text}

$n_{Y}=2, n_{Z}=3$ invariants with self-loops

$$
\begin{aligned}
& Y_{a_{7}}^{a_{1}} Y_{a_{5}}^{a_{2}} Z_{a_{3} a_{8}}^{a_{3} a_{4}} Z_{a_{4} a_{6}}^{a_{5} a_{6}} Z_{a_{1} a_{2}}^{a_{7} a_{8}} \\
& Y_{a_{7}}^{a_{1}} Y_{a_{5}}^{a_{2}} Z_{a_{3} a_{6}}^{a_{3} a_{4}} Z_{a_{4} a_{8}}^{a_{5} a_{6}} Z_{a_{1} a_{2}}^{a_{7} a_{8}} \\
& Y_{a_{5}}^{a_{1}} Y_{a_{3}}^{a_{2}} Z_{a_{7} a_{8}}^{a_{3} a_{4}} Z_{a_{4} a_{6}}^{a_{5} a_{6}} Z_{a_{1} a_{2}}^{a_{7} a_{8}} \\
& Y_{a_{5}}^{a_{1}} Y_{a_{3}}^{a_{2}} Z_{a_{4} a_{7}}^{a_{3} a_{4}} Z_{a_{5} a_{6}}^{a_{1}} Z_{a_{7} a_{8}}^{a_{1}} \\
& Y_{a_{5}} Y_{a_{3}} Z_{a_{4} a_{7}}^{a_{a_{6}} a_{8}} Z_{a_{1} a_{2}}^{a_{4} a_{6}} \\
& Y_{a_{7}}^{a_{1}} Y_{a_{3}}^{a_{2}} Z_{a_{5} a_{8}}^{a_{3} a_{4}} Z_{a_{2} a_{6}}^{a_{5} a_{6}} Z_{a_{1} a_{4}}^{a_{a_{1}} a_{8}} \\
& Y_{a_{5}}^{a_{1}} Y_{a_{3}}^{a_{2}} Z_{a_{6} a_{7}}^{a_{3} a_{4}} Z_{a_{2} a_{4}}^{a_{5} a_{6}} Z_{a_{1} a_{8}}^{a_{a_{1}} a_{8}} \\
& Y_{a_{2}}^{a_{1}} Y_{a_{5}}^{a_{2}} Z_{a_{7} a_{8}}^{a_{3} a_{4}} Z_{a_{3} a_{6}}^{a_{5} a_{6}} Z_{a_{1} a_{4}}^{a_{7} a_{8}} \\
& Y_{a_{2}}^{a_{1}} Y_{a_{5}}^{a_{2}} Z_{a_{3} a_{7}}^{a_{3} a_{4}} Z_{a_{6} a_{8}}^{a_{5} a_{6}} Z_{a_{1} a_{4}}^{a_{7} a_{8}}
\end{aligned}
$$


$n_{Y}=3, n_{Z}=3$ Invariants with self-loops

$$
\begin{aligned}
& Y_{a_{8}}^{a_{1}} Y_{a_{6}}^{a_{2}} Y_{a_{4}}^{a_{3}} Z_{a_{5} a_{9}}^{a_{4} a_{5}} Z_{a_{3} a_{7}}^{a_{6} a_{7}} Z_{a_{1} a_{2}}^{a_{8} a_{9}} \\
& Y_{a_{8}}^{a_{1}} Y_{a_{6}}^{a_{2}} Y_{a_{4}}^{a_{3}} Z_{a_{5} a_{7}}^{a_{4} a_{5}} Z_{a_{3} a_{9}}^{a_{6} a_{7}} Z_{a_{1} a_{2}}^{a_{8} a_{9}} \\
& Y_{a_{6}}^{a_{1}} Y_{a_{7}}^{a_{2}} Y_{a_{4}}^{a_{3}} Z_{a_{5} a_{5}}^{a_{4} a_{5}} Z_{a_{3} a_{9}}^{a_{6} a_{7}} Z_{a_{1} a_{2}}^{a_{a_{9}}} \\
& Y_{a_{8}}^{a_{1}} Y_{a_{4}}^{a_{2}} Y_{a_{6}}^{a_{3}} Z_{a_{5} a_{9}}^{a_{4} a_{5}} Z_{a_{3} a_{7}}^{a_{6} a_{7}} Z_{a_{1} a_{2}}^{a_{8} a_{9}} \\
& Y_{a_{8}}^{a_{1}} Y_{a_{4}}^{a_{2}} Y_{a_{6}}^{a_{3}} Z_{a_{5} a_{7}}^{a_{4} a_{5}} Z_{a_{3} a_{9}}^{a_{6} a_{7}} Z_{a_{1} a_{2}}^{a_{8} a_{9}} \\
& Y_{a_{6}}^{a_{1}} Y_{a_{4}}^{a_{2}} Y_{a_{8}}^{a_{3}} Z_{a_{5} a_{9}}^{a_{4} a_{5}} Z_{a_{3} a_{7}}^{a_{6} a_{7}} Z_{a_{1} a_{2}}^{a_{8} a_{9}} \\
& Y_{a_{6}}^{a_{1}} Y_{a_{4}}^{a_{2}} Y_{a_{8}}^{a_{3}} Z_{a_{5} a_{7}}^{a_{4} a_{5}} Z_{a_{3} a_{9}}^{a_{6} a_{7}} Z_{a_{1} a_{2}}^{a_{8} a_{9}} \\
& Y_{a_{6}}^{a_{1}} Y_{a_{4}}^{a_{2}} Y_{a_{7}}^{a_{3}} Z_{a_{5} a_{8}}^{a_{4} a_{5}} Z_{a_{3} a_{9}}^{a_{6} a_{7}} Z_{a_{1} a_{2}}^{a_{8} a_{9}} \\
& Y_{a_{8}}^{a_{1}} Y_{a_{3}}^{a_{2}} Y_{a_{6}}^{a_{3}} Z_{a_{4} a_{9}}^{a_{4} a_{5}} Z_{a_{5} a_{7}}^{a_{6} a_{7}} Z_{a_{1} a_{2}}^{a_{8} a_{9}} \\
& Y_{a_{8}}^{a_{1}} Y_{a_{3}}^{a_{2}} Y_{a_{6}}^{a_{3}} Z_{a_{4} a_{7}}^{a_{4} a_{5}} Z_{a_{5} a_{7}}^{a_{6} a_{7}} Z_{a_{1} a_{9}}^{a_{2}} \\
& Y^{a_{1}} Y^{a_{2}} Y^{a_{3}} Z^{a_{4} a_{5}} Z^{a_{6} a_{7}} Z^{a_{8} a_{9}} \\
& Y_{a_{6}}^{a_{1}} Y_{a_{3}}^{a_{2}} Y_{a_{8}}^{a_{3}} Z_{a_{4} a_{9}}^{a_{4} a_{5}} Z_{a_{5} a_{7}}^{a_{6} a_{7}} Z_{a_{1} a_{2}}^{a_{8} a_{9}} \\
& Y_{a_{6}}^{a_{1}} Y_{a_{3}}^{a_{2}} Y_{a_{8}}^{a_{3}} Z_{a_{4} a_{7}}^{a_{4} a_{5}} Z_{a_{5} a_{9}}^{a_{6} a_{7}} Z_{a_{1} a_{2}}^{a_{8} a_{9}} \\
& Y_{a_{6}}^{a_{1}} Y_{a_{3}}^{a_{2}} Y_{a_{4}}^{a_{3}} Z_{a_{8} a_{9}}^{a_{4} a_{5}} Z_{a_{5} a_{7}}^{a_{6} a_{7}} Z_{a_{1} a_{2}}^{a_{8} a_{9}} \\
& Y_{a_{6}}^{a_{1}} Y_{a_{3}}^{a_{2}} Y_{a_{4}}^{a_{3}} Z_{a_{5} a_{5}}^{a_{4} a_{5}} Z_{a_{7} a_{9}}^{a_{6} a_{7}} Z_{a_{1} a_{2}}^{a_{8} a_{9}} \\
& Y_{a_{6}}^{a_{1}} Y_{a_{3}}^{a_{2}} Y_{a_{4}}^{a_{3}} Z_{a_{5} a_{7}}^{a_{4} a_{5}} Z_{a_{8} a_{9}}^{a_{6} a_{7}} Z_{a_{1} a_{2}}^{a_{8} a_{9}} \\
& Y_{a_{8}}^{a_{1}} Y_{a_{4}}^{a_{2}} Y_{a_{3}}^{a_{3}} Z_{a_{6} a_{9}}^{a_{4} a_{5}} Z_{a_{2} a_{7}}^{a_{6} a_{7}} Z_{a_{1} a_{5}}^{a_{8} a_{9}} \\
& Y_{a_{6}}^{a_{1}} Y_{a_{4}}^{a_{2}} Y_{a_{3}}^{a_{3}} Z_{a_{7} a_{8}}^{a_{4} a_{5}} Z_{a_{2} a_{5}}^{a_{6} a_{7}} Z_{a_{1} a_{9}}^{a_{8} a_{9}} \\
& Y_{a_{8}}^{a_{1}} Y_{a_{3}}^{a_{2}} Y_{a_{6}}^{a_{3}} Z_{a_{4} a_{7}}^{a_{4} a_{5}} Z_{a_{2} a_{9}}^{a_{6} a_{7}} Z_{a_{1} a_{5}}^{a_{8} a_{9}} \\
& Y_{a_{6}}^{a_{1}} Y_{a_{3}}^{a_{2}} Y_{a_{8}}^{a_{3}} Z_{a_{4} a_{9}}^{a_{4} a_{5}} Z_{a_{2} a_{7}}^{a_{6} a_{7}} Z_{a_{1} a_{5}}^{a_{8} a_{9}} \\
& Y_{a_{8}}^{a_{1}} Y_{a_{3}}^{a_{2}} Y_{a_{4}}^{a_{3}} Z_{a_{6} a_{9}}^{a_{4} a_{5}} Z_{a_{2} a_{7}}^{a_{6} a_{7}} Z_{a_{1} a_{5}}^{a_{8} a_{9}} \\
& Y_{a_{6}}^{a_{1}} Y_{a_{3}}^{a_{2}} Y_{a_{4}}^{a_{3}} Z_{a_{8} a_{9}}^{a_{4} a_{5}} Z_{a_{2} a_{7}}^{a_{6} a_{7}} Z_{a_{1} a_{5}}^{a_{8} a_{9}} \\
& Y_{a_{6}}^{a_{1}} Y_{a_{3}}^{a_{2}} Y_{a_{4}}^{a_{3}} Z_{a_{7} a_{8}}^{a_{4} a_{5}} Z_{a_{2} a_{5}}^{a_{6} a_{7}} Z_{a_{1} a_{9}}^{a_{8} a_{9}} \\
& Y_{a_{6}}^{a_{1}} Y_{a_{3}}^{a_{2}} Y_{a_{4}}^{a_{3}} Z_{a_{5} a_{8}}^{a_{4} a_{5}} Z_{a_{2} a_{9}}^{a_{6} a_{7}} Z_{a_{1} a_{7}}^{a_{8} a_{9}} \\
& Y_{a_{6}}^{a_{1}} Y_{a_{3}}^{a_{2}} Y_{a_{4}}^{a_{3}} Z_{a_{5} a_{8}}^{a_{4} a_{5}} Z_{a_{2} a_{7}}^{a_{6} a_{7}} Z_{a_{1} a_{9}}^{a_{8} a_{9}} \\
& Y_{a_{6}}^{a_{1}} Y_{a_{3}}^{a_{2}} Y_{a_{4}}^{a_{3}} Z_{a_{5} a_{7}}^{a_{4} a_{5}} Z_{a_{2} a_{8}}^{a_{6} a_{7}} Z_{a_{1} a_{9}}^{a_{8} a_{9}} \\
& Y_{a_{4}}^{a_{1}} Y_{a_{3}}^{a_{2}} Y_{a_{6}}^{a_{3}} Z_{a_{7} a_{8}}^{a_{4} a_{5}} Z_{a_{2} a_{5}}^{a_{6} a_{7}} Z_{a_{1} a_{9}}^{a_{8} a_{9}} \\
& Y_{a_{6}}^{a_{1}} Y_{a_{3}}^{a_{2}} Y_{a_{2}}^{a_{3}} Z_{a_{8} a_{9}}^{a_{4} a_{5}} Z_{a_{4} a_{7}}^{a_{6} a_{7}} Z_{a_{1} a_{5}}^{a_{8} a_{9}} \\
& Y_{a_{6}}^{a_{1}} Y_{a_{3}}^{a_{2}} Y_{a_{2}}^{a_{3}} Z_{a_{4} a_{8}}^{a_{4} a_{5}} Z_{a_{7} a_{9}}^{a_{6} a_{7}} Z_{a_{1} a_{5}}^{a_{a_{9}}} \\
& Y_{a_{3}}^{a_{1}} Y_{a_{6}}^{a_{2}} Y_{a_{2}}^{a_{3}} Z_{a_{8} a_{9}}^{a_{4} a_{5}} Z_{a_{4} a_{7}}^{a_{6} a_{7}} Z_{a_{1} a_{5}}^{a_{8} a_{9}} \\
& Y_{a_{3}}^{a_{1}} Y_{a_{6}}^{a_{2}} Y_{a_{2}}^{a_{3}} Z_{a_{4} a_{8}}^{a_{4} a_{5}} Z_{a_{7} a_{9}}^{a_{6} a_{7}} Z_{a_{1} a_{5}}^{a_{8} a_{9}}
\end{aligned}
$$


$n_{y}=1, n_{Z}=4$ Invariants with self-loops

$$
\begin{aligned}
& Y_{a_{8}}^{a_{1}} Z_{a_{6} a_{7}}^{a_{2} a_{3}} Z_{a_{4} a_{9}}^{a_{4} a_{5}} Z_{a_{2} a_{5}}^{a_{6} a_{7}} Z_{a_{1} a_{3}}^{a_{8} a_{9}} \\
& Y_{a_{6}}^{a_{1}} Z^{a_{2} a_{3}} Z^{a_{4} a_{5}} Z^{a_{6} a_{7}} Z^{a_{8} a_{9}} \\
& Y^{a_{1}} Z^{a_{2} a_{3}} Z^{a_{4} a_{5}} Z^{a_{6} a_{7}} Z^{a_{8} a_{9}} \\
& Y_{a_{6}}^{a_{1}} Z_{a_{4} a_{8}}^{a_{2} a_{3}} Z_{a_{5} a_{9}}^{a_{4} a_{5}} Z_{a_{2} a_{7}}^{a_{6} a_{7}} Z_{a_{1} a_{3}}^{a_{8} a_{9}} \\
& Y^{a_{1}} Z_{a_{2} a_{3}}^{a_{2}} Z^{a_{4} a_{5}} Z^{a_{6} a_{7}} Z^{a_{8} a_{9}} \\
& Y_{a_{6}} Z_{a_{4} a_{8}} Z_{a_{5} a_{7}}^{a_{a_{2} a_{9}}} Z_{a_{1} a_{3}} \\
& Y_{a_{6}}^{a_{1}} Z_{a_{4} a_{7}}^{a_{2} a_{3}} Z_{a_{5} a_{8}}^{a_{4} a_{5}} Z_{a_{2} a_{9}}^{a_{6} a_{7}} Z_{a_{1} a_{3}}^{a_{a_{9}} a_{9}} \\
& Y_{a_{6}}^{a_{1}} Z_{a_{4} a_{5}}^{a_{2} a_{3}} Z_{a_{8} a_{9}}^{a_{4} a_{5}} Z_{a_{2} a_{7}}^{a_{6} a_{7}} Z_{a_{1} a_{3}}^{a_{8} a_{9}} \\
& Y_{a_{4}}^{a_{1}} Z_{a_{8} a_{9}}^{a_{2} a_{3}} Z_{a_{5} a_{6}}^{a_{4} a_{5}} Z_{a_{2} a_{7}}^{a_{6} a_{7}} Z_{a_{1} a_{3}}^{a_{8} a_{9}} \\
& Y_{a_{4}}^{a_{1}} Z_{a_{6} a_{8}}^{a_{2} a_{3}} Z_{a_{5} a_{9}}^{a_{4} a_{5}} Z_{a_{2} a_{7}}^{a_{6} a_{7}} Z_{a_{1} a_{3}}^{a_{a_{3}} a_{9}} \\
& Y_{a_{4}}^{a_{1}} Z_{a_{6} a_{8}}^{a_{2} a_{3}} Z_{a_{5} a_{7}}^{a_{4} a_{5}} Z_{a_{2} a_{9}}^{a_{6} a_{7}} Z_{a_{1} a_{3}}^{a_{8} a_{9}} \\
& Y_{a_{1}}^{a_{1}} Z^{a_{2} a_{3}} Z^{a_{4} a_{5}} Z^{a_{6} a_{7}} Z^{a_{8} a_{9}} \\
& Y_{a_{6}}^{a_{1}} Z_{a_{4} a_{8}}^{a_{2} a_{3}} Z_{a_{5} a_{9}}^{a_{4} a_{5}} Z_{a_{2} a_{3}}^{a_{6} a_{7}} Z_{a_{1} a_{7}}^{a_{1} a_{9}} \\
& Y_{a_{6}}^{a_{1}} Z_{a_{4} a_{8}}^{a_{2} a_{3}} Z_{a_{5} a_{7}}^{a_{4} a_{5}} Z_{a_{2} a_{3}}^{a_{6} a_{7}} Z_{a_{1} a_{9}}^{a_{1} a_{9}} \\
& Y_{a_{6}}^{a_{1}} Z_{a_{2} a_{8}}^{a_{2} a_{3}} Z_{a_{4} a_{9}}^{a_{4} a_{5}} Z_{a_{5} a_{7}}^{a_{6} a_{7}} Z_{a_{1} a_{3}}^{a_{8} a_{9}} \\
& Y_{a_{6}}^{a_{1}} Z_{a_{2} a_{4}}^{a_{2} a_{3}} Z_{a_{5} a_{8}}^{a_{4} a_{5}} Z_{a_{7} a_{9}}^{a_{6} a_{7}} Z_{a_{1} a_{3}}^{a_{8} a_{9}}
\end{aligned}
$$

\section{B.5 Lists of spontaneous CP-odd invariants}

$n_{v}=1, n_{Z}=3$

$$
\begin{aligned}
& v_{a_{4}} v^{* a_{1}} Z_{a_{6} a_{7}}^{a_{2} a_{3}} Z_{a_{2} a_{5}}^{a_{4} a_{5}} Z_{a_{1} a_{3}}^{a_{6} a_{7}} \\
& v_{a_{4}} v^{* a_{1}} Z_{a_{2} a_{6}}^{a_{2} a_{3}} Z_{a_{5} a_{7}}^{a_{4} a_{5}} Z_{a_{1} a_{3}}^{a_{6} a_{7}}
\end{aligned}
$$

$n_{v}=2, n_{Z}=3$

$$
\begin{aligned}
& v_{a_{5}} v_{a_{7}} v^{* a_{1}} v^{* a_{2}} Z_{a_{6} a_{8}}^{a_{3} a_{4}} Z_{a_{3} a_{4}}^{a_{5} a_{6}} Z_{a_{1} a_{2}}^{a_{7} a_{8}} \\
& v_{a_{5}} v_{a_{7}} v^{* a_{1}} v^{* a_{2}} Z_{a_{3} a_{8}}^{a_{3} a_{4}} Z_{a_{4} a_{6}}^{a_{5} a_{6}} Z_{a_{1} a_{2}}^{a_{7} a_{8}} \\
& v_{a_{5}} v_{a_{7}} v^{* a_{1}} v^{* a_{2}} Z_{a_{3} a_{6}}^{a_{3} a_{4}} Z_{a_{4} a_{8}}^{a_{5} a_{6}} Z_{a_{1} a_{2}}^{a_{7} a_{8}} \\
& v_{a_{5}} v_{a_{7}} v^{* a_{1}} v^{* a_{2}} Z_{a_{3} a_{4}}^{a_{3} a_{4}} Z_{a_{6} a_{8}}^{a_{5} a_{6}} Z_{a_{1} a_{2}}^{a_{7} a_{8}} \\
& v_{a_{3}} v_{a_{5}} v^{* a_{1}} v^{* a_{2}} Z_{a_{7} a_{8}}^{a_{3} a_{4}} Z_{a_{4} a_{6}}^{a_{5} a_{6}} Z_{a_{1} a_{2}}^{a_{7} a_{8}} \\
& v_{a_{3}} v_{a_{5}} v^{* a_{1}} v^{* a_{2}} Z_{a_{6} a_{7}}^{a_{3} a_{4}} Z_{a_{4} a_{8}}^{a_{5} a_{6}} Z_{a_{1} a_{2}}^{a_{7} a_{8}} \\
& v_{a_{3}} v_{a_{5}} v^{* a_{1}} v^{* a_{2}} Z_{a_{4} a_{7}}^{a_{3} a_{4}} Z_{a_{6} a_{8}}^{a_{5} a_{6}} Z_{a_{1} a_{2}}^{a_{7} a_{8}} \\
& v_{a_{3}} v_{a_{7}} v^{* a_{1}} v^{* a_{2}} Z_{a_{5} a_{8}}^{a_{3} a_{4}} Z_{a_{1} a_{6}}^{a_{5} a_{6}} Z_{a_{2} a_{4}}^{a_{7} a_{8}}
\end{aligned}
$$




$$
n_{v}=1, n_{Z}=4
$$

$$
\begin{aligned}
& v_{a_{8}} v^{* a_{1}} Z_{a_{6} a_{7}}^{a_{2} a_{3}} Z_{a_{4} a_{9}}^{a_{4} a_{5}} Z_{a_{2} a_{5}}^{a_{6} a_{7}} Z_{a_{1} a_{3}}^{a_{8} a_{9}} \\
& v_{a_{6}} v^{* a_{1}} Z_{a_{8} a_{9}}^{a_{2} a_{3}} Z_{a_{4} a_{7}}^{a_{4} a_{5}} Z_{a_{2} a_{5}}^{a_{6} a_{7}} Z_{a_{1} a_{3}}^{a_{8} a_{9}} \\
& v_{a_{6}} v^{* a_{1}} Z_{a_{4} a_{7}}^{a_{2} a_{3}} Z_{a_{8} a_{9}}^{a_{4} a_{5}} Z_{a_{2} a_{5}}^{a_{6} a_{7}} Z_{a_{1} a_{3}}^{a_{8} a_{9}} \\
& v_{a_{8}} v^{* a_{1}} Z_{a_{4} a_{6}}^{a_{2} a_{3}} Z_{a_{5} a_{9}}^{a_{4} a_{5}} Z_{a_{2} a_{7}}^{a_{6} a_{7}} Z_{a_{1} a_{3}}^{a_{8} a_{9}} \\
& v_{a_{6}} v^{* a_{1}} Z_{a_{4} a_{8}}^{a_{2} a_{3}} Z_{a_{5} a_{9}}^{a_{4} a_{5}} Z_{a_{2} a_{7}}^{a_{6} a_{7}} Z_{a_{1} a_{3}}^{a_{a_{9}} a_{9}} \\
& v_{a_{6}} v^{* a_{1}} Z_{a_{4} a_{8}}^{a_{2} a_{3}} Z_{a_{5} a_{7}}^{a_{4} a_{5}} Z_{a_{2} a_{9}}^{a_{6} a_{7}} Z_{a_{1} a_{3}}^{a_{8} a_{9}} \\
& v_{a_{6}} v^{* a_{1}} Z_{a_{4} a_{7}}^{a_{2} a_{3}} Z_{a_{5} a_{8}}^{a_{4} a_{5}} Z_{a_{2} a_{9}}^{a_{6} a_{7}} Z_{a_{1} a_{3}}^{a_{8} a_{9}} \\
& v_{a_{6}} v^{* a_{1}} Z_{a_{4} a_{5}}^{a_{2} a_{3}} Z_{a_{8} a_{9}}^{a_{4} a_{5}} Z_{a_{2} a_{7}}^{a_{6} a_{7}} Z_{a_{1} a_{3}}^{a_{8} a_{9}} \\
& v_{a_{4}} v^{* a_{1}} Z_{a_{8} a_{9}}^{a_{2} a_{3}} Z_{a_{5} a_{6}}^{a_{4} a_{5}} Z_{a_{2} a_{7}}^{a_{6} a_{7}} Z_{a_{1} a_{3}}^{a_{a_{9}} a_{9}} \\
& v_{a_{4}} v^{* a_{1}} Z_{a_{6} a_{8}}^{a_{2} a_{3}} Z_{a_{5} a_{9}}^{a_{4} a_{5}} Z_{a_{2} a_{7}}^{a_{6} a_{7}} Z_{a_{1} a_{3}}^{a_{8} a_{9}} \\
& v_{a_{4}} v^{* a_{1}} Z_{a_{6} a_{8}}^{a_{2} a_{3}} Z_{a_{5} a_{7}}^{a_{4} a_{5}} Z_{a_{2} a_{9}}^{a_{6} a_{7}} Z_{a_{1} a_{3}}^{a_{1} a_{9}} \\
& v_{a_{4}} v^{* a_{1}} Z_{a_{6} a_{7}}^{a_{2} a_{3}} Z_{a_{5} a_{8}}^{a_{4} a_{5}} Z_{a_{2} a_{9}}^{a_{6} a_{7}} Z_{a_{1} a_{3}}^{a_{a_{9}}} \\
& v_{a_{6}} v^{* a_{1}} Z_{a_{4} a_{8}}^{a_{2} a_{3}} Z_{a_{5} a_{9}}^{a_{4} a_{5}} Z_{a_{2} a_{3}}^{a_{6} a_{7}} Z_{a_{1} a_{7}}^{a_{8} a_{9}} \\
& v_{a_{6}} v^{* a_{1}} Z_{a_{4} a_{8}}^{a_{2} a_{3}} Z_{a_{5} a_{7}}^{a_{4} a_{5}} Z_{a_{2} a_{3}}^{a_{6} a_{7}} Z_{a_{1} a_{9}}^{a_{a_{9}} a_{9}} \\
& v_{a_{6}} v^{* a_{1}} Z_{a_{2} a_{8}}^{a_{2} a_{3}} Z_{a_{4} a_{9}}^{a_{4} a_{5}} Z_{a_{5} a_{7}}^{a_{6} a_{7}} Z_{a_{1} a_{3}}^{a_{8} a_{9}} \\
& v_{a_{6}} v^{* a_{1}} Z_{a_{2} a_{4}}^{a_{2} a_{3}} Z_{a_{5} a_{8}}^{a_{4} a_{5}} Z_{a_{7} a_{9}}^{a_{6} a_{7}} Z_{a_{1} a_{3}}^{a_{a_{3}} a_{9}}
\end{aligned}
$$

\section{B.6 Larger CP-odd invariants}

In addition to the smaller invariants discussed previously, we also found some larger CPIs with up to $9 Z$ tensors. Using the notation established in Eq. 2.40), we show only half of the CPI, which is sufficient to uniquely define it.

$$
\begin{aligned}
I_{1}^{(7,2)} & =Z_{b_{1} b_{2}}^{b_{3} b_{4}} Z_{b_{3} a_{1}}^{b_{1} a_{5}} Z_{b_{4} a_{2}}^{b_{2} a_{6}} Z_{c_{1} c_{2}}^{c_{3} c_{4}} Z_{c_{3} a_{3}}^{c_{1} a_{1}^{\prime}} Z_{c_{4} a_{4}}^{c_{2} a_{2}} Z_{a_{5} a_{6}}^{a_{3}^{\prime} a_{4}} Y_{a_{1}^{\prime}}^{a_{1}} Y_{a_{3}^{\prime}}^{a_{3}}, \\
I_{1}^{(8)} & =Z_{b_{1} b_{2}}^{b_{3} b_{4}} Z_{b_{3} b_{5}}^{b_{1} b_{6}} Z_{b_{4} a_{1}}^{b_{2} a_{5}} Z_{b_{6} a_{2}}^{b_{5} a_{6}} Z_{c_{1} c_{2}}^{c_{3} c_{4}} Z_{c_{3} a_{3}}^{c_{1} a_{1}} Z_{c_{4} a_{4}}^{c_{2} a_{2}} Z_{a_{5} a_{6}}^{a_{3} a_{4}}, \\
I_{1}^{(9)} & =Z_{b_{1} b_{2}}^{b_{3} b_{4}} Z_{b_{3} b_{5}}^{b_{1} b_{6}} Z_{b_{4} b_{7}}^{b_{2} b_{8}} Z_{b_{6} a_{1}}^{b_{5} a_{5}} Z_{b_{8} a_{2}}^{b_{7} a_{6}} Z_{c_{1} c_{2}}^{c_{3} c_{4}} Z_{c_{3} a_{3}}^{c_{1} a_{1}} Z_{c_{4} a_{4}}^{c_{2} a_{2}} Z_{a_{5} a_{6}}^{a_{3} a_{4}}
\end{aligned}
$$

The respective CPIs $\mathcal{I}_{1}^{(7,2)}, \mathcal{I}_{1}^{(8)}, \mathcal{I}_{1}^{(9)}$ can be obtained by subtracting from the $I$ above the $I^{*}$ obtained by swapping the upper and lower indices, as described in general in Section 2.2. 


\section{C $\Delta\left(6 n^{2}\right)$ potentials as particular cases of $\Delta\left(3 n^{2}\right)$ po- tentials}

The triplet generators of $\Delta\left(6 n^{2}\right)$ with $n \in \mathbb{N}$ are 80

$$
a=\left(\begin{array}{ccc}
0 & 1 & 0 \\
0 & 0 & 1 \\
1 & 0 & 0
\end{array}\right), \quad b= \pm\left(\begin{array}{ccc}
0 & 0 & 1 \\
0 & 1 & 0 \\
1 & 0 & 0
\end{array}\right), \quad c=\left(\begin{array}{ccc}
\eta^{l} & 0 & 0 \\
0 & \eta^{-l} & 0 \\
0 & 0 & 1
\end{array}\right)
$$

where $\eta=e^{2 \pi i / n}$ and $l \in \mathbb{N}$. If the field transforms as a faithful triplet, any $c$-invariant operator $\mathcal{O}$ will also be invariant under the phase transformation 11

$$
c_{0}=\left(\begin{array}{ccc}
\eta & 0 & 0 \\
0 & \eta^{-1} & 0 \\
0 & 0 & 1
\end{array}\right)
$$

and therefore also under

$$
c_{0}^{-l}=\left(\begin{array}{ccc}
\eta^{-l} & 0 & 0 \\
0 & \eta^{l} & 0 \\
0 & 0 & 1
\end{array}\right) \quad \text { and } \quad c_{0}^{k}=\left(\begin{array}{ccc}
\eta^{k} & 0 & 0 \\
0 & \eta^{-k} & 0 \\
0 & 0 & 1
\end{array}\right)
$$

Imposing additionally invariance under $a$, we quickly find that the operator $\mathcal{O}$ is also invariant under

$$
a c_{0}^{-l} a^{2}=\left(\begin{array}{ccc}
\eta^{l} & 0 & 0 \\
0 & 1 & 0 \\
0 & 0 & \eta^{-l}
\end{array}\right) \quad \text { and } \quad a^{2} c_{0}^{k} a=\left(\begin{array}{ccc}
1 & 0 & 0 \\
0 & \eta^{k} & 0 \\
0 & 0 & \eta^{-k}
\end{array}\right)
$$

As a result, the operator $\mathcal{O}$ is symmetric under the successive application of $a c_{0}^{-l} a^{2}$ and $a^{2} c_{0}^{k} a$, i.e.

$$
\left(\begin{array}{ccc}
\eta^{l} & 0 & 0 \\
0 & \eta^{k} & 0 \\
0 & 0 & \eta^{-k-l}
\end{array}\right)
$$

Demanding invariance under $a$ and $c$ of Eq. (C.1) therefore leads to the set of $\Delta\left(3 n^{2}\right)$ invariant operators where the triplet generators are given by [78]

$$
a^{\prime}=\left(\begin{array}{ccc}
0 & 1 & 0 \\
0 & 0 & 1 \\
1 & 0 & 0
\end{array}\right), \quad c^{\prime}=\left(\begin{array}{ccc}
\eta^{l} & 0 & 0 \\
0 & \eta^{k} & 0 \\
0 & 0 & \eta^{-k-l}
\end{array}\right)
$$

We thus conclude that the $\Delta\left(6 n^{2}\right)$ symmetric potential can be deduced from the $\Delta\left(3 n^{2}\right)$ invariant potential by simply dropping all terms which are not symmetric under $b$ of Eq. C.1. Therefore, in each of these cases it is sufficient to use the already obtained expressions for the CPIs and set constraints on the coefficients to make all the terms in the potential invariant under the $b$ generator.

\footnotetext{
${ }^{11}$ For faithful representations, $l$ and $n$ have to be coprime. As a consequence, there must be an integer $p$ such that $c^{p}=c_{0}$.
} 


\section{References}

[1] M. Kobayashi and T. Maskawa, Prog. Theor. Phys. 49 (1973) 652.

[2] C. Jarlskog, Phys. Rev. Lett. 55 (1985) 1039.

[3] G. Aad et al. [ATLAS Collaboration], Phys. Lett. B 716 (2012) 1 [arXiv:1207.7214].

[4] S. Chatrchyan et al. [CMS Collaboration], Phys. Lett. B 716 (2012) 30 [arXiv:1207.7235].

[5] T. D. Lee, Phys. Rev. D 8 (1973) 1226.

[6] A. D. Sakharov, Pisma Zh. Eksp. Teor. Fiz. 5 (1967) 32 [JETP Lett. 5 (1967) 24] [Sov. Phys. Usp. 34 (1991) 392] [Usp. Fiz. Nauk 161 (1991) 61].

[7] V. A. Kuzmin, V. A. Rubakov and M. E. Shaposhnikov, Phys. Lett. B 155 (1985) 36.

[8] D. V. Forero, M. Tortola and J. W. F. Valle, Phys. Rev. D 90 (2014) no.9, 093006 [arXiv:1405.7540].

[9] M. C. Gonzalez-Garcia, M. Maltoni and T. Schwetz, JHEP 1411 (2014) 052 [arXiv:1409.5439].

[10] M. Fukugita and T. Yanagida, Phys. Lett. B 174 (1986) 45.

[11] G. Altarelli and F. Feruglio, Rev. Mod. Phys. 82 (2010) 2701 [arXiv:1002.0211].

[12] H. Ishimori, T. Kobayashi, H. Ohki, Y. Shimizu, H. Okada and M. Tanimoto, Prog. Theor. Phys. Suppl. 183 (2010) 1 [arXiv:1003.3552].

[13] W. Grimus and P. O. Ludl, J. Phys. A 45 (2012) 233001 [arXiv:1110.6376].

[14] S. F. King and C. Luhn, Rept. Prog. Phys. 76 (2013) 056201 [arXiv:1301.1340].

[15] S. F. King, A. Merle, S. Morisi, Y. Shimizu and M. Tanimoto, New J. Phys. 16 (2014) 045018 [arXiv:1402.4271].

[16] S. F. King, J. Phys. G 42 (2015) 123001 [arXiv:1510.02091].

[17] J. Bernabeu, G. C. Branco and M. Gronau, Phys. Lett. B 169 (1986) 243.

[18] G. C. Branco, L. Lavoura and M. N. Rebelo, Phys. Lett. B 180 (1986) 264.

[19] G. C. Branco, I. de Medeiros Varzielas and S. F. King, Phys. Rev. D 92 (2015) no.3, 036007 [arXiv:1502.03105].

[20] G. C. Branco, I. de Medeiros Varzielas and S. F. King, Nucl. Phys. B 899 (2015) 14 [arXiv:1505.06165].

[21] V. Keus, S. F. King, S. Moretti and K. Yagyu, arXiv:1510.04028. 
[22] A. Mendez and A. Pomarol, Phys. Lett. B 272 (1991) 313.

[23] L. Lavoura and J. P. Silva, Phys. Rev. D 50 (1994) 4619 [hep-ph/9404276].

[24] F. J. Botella and J. P. Silva, Phys. Rev. D 51 (1995) 3870 [hep-ph/9411288].

[25] G. C. Branco, M. N. Rebelo and J. I. Silva-Marcos, Phys. Lett. B 614 (2005) 187 [hep-ph/0502118].

[26] S. Davidson and H. E. Haber, Phys. Rev. D 72 (2005) 035004 Erratum: [Phys. Rev. D 72 (2005) 099902] [hep-ph/0504050].

[27] J. F. Gunion and H. E. Haber, Phys. Rev. D 72 (2005) 095002 [hep-ph/0506227].

[28] F. Feruglio, C. Hagedorn and R. Ziegler, JHEP 1307 (2013) 027 [arXiv:1211.5560].

[29] G. J. Ding, S. F. King, C. Luhn and A. J. Stuart, JHEP 1305 (2013) 084 [arXiv:1303.6180].

[30] F. Feruglio, C. Hagedorn and R. Ziegler, Eur. Phys. J. C 74 (2014) 2753 [arXiv:1303.7178].

[31] S. F. King, T. Neder and A. J. Stuart, Phys. Lett. B 726 (2013) 312 [arXiv:1305.3200].

[32] C. Luhn, Nucl. Phys. B 875 (2013) 80 [arXiv:1306.2358].

[33] G. J. Ding, S. F. King and A. J. Stuart, JHEP 1312 (2013) 006 [arXiv:1307.4212].

[34] C. C. Li and G. J. Ding, Nucl. Phys. B 881 (2014) 206 [arXiv:1312.4401].

[35] G. J. Ding and Y. L. Zhou, Chin. Phys. C 39 (2015) no.2, 021001 [arXiv:1312.5222].

[36] S. F. King and T. Neder, Phys. Lett. B 736 (2014) 308 [arXiv:1403.1758].

[37] G. J. Ding and S. F. King, Phys. Rev. D 89 (2014) no.9, 093020 [arXiv:1403.5846].

[38] G. J. Ding and Y. L. Zhou, JHEP 1406 (2014) 023 [arXiv:1404.0592].

[39] T. Neder, J. Phys. Conf. Ser. 598 (2015) no.1, 012025 [arXiv:1405.0019].

[40] C. C. Li and G. J. Ding, JHEP 1508 (2015) 017 [arXiv:1408.0785].

[41] C. Hagedorn, A. Meroni and E. Molinaro, Nucl. Phys. B 891 (2015) 499 [arXiv:1408.7118].

[42] G. J. Ding, S. F. King and T. Neder, JHEP 1412 (2014) 007 [arXiv:1409.8005].

[43] F. Björkeroth, F. J. de Anda, I. de Medeiros Varzielas and S. F. King, JHEP 1506 (2015) 141 [arXiv:1503.03306].

[44] C. C. Li and G. J. Ding, JHEP 1505 (2015) 100 [arXiv:1503.03711]. 
[45] A. Di Iura, C. Hagedorn and D. Meloni, JHEP 1508 (2015) 037 [arXiv:1503.04140].

[46] P. Ballett, S. Pascoli and J. Turner, Phys. Rev. D 92 (2015) no.9, 093008 [arXiv:1503.07543].

[47] T. Neder, J. Phys. Conf. Ser. 631 (2015) no.1, 012019 [arXiv:1503.09041].

[48] J. Turner, Phys. Rev. D 92 (2015) no.11, 116007 [arXiv:1507.06224].

[49] G. J. Ding and S. F. King, Phys. Rev. D 93 (2016) 025013 [arXiv:1510.03188].

[50] F. Björkeroth, F. J. de Anda, I. de Medeiros Varzielas and S. F. King, arXiv:1512.00850.

[51] C. C. Li, C. Y. Yao and G. J. Ding, arXiv:1601.06393.

[52] G. C. Branco, J. M. Gerard and W. Grimus, Phys. Lett. B 136 (1984) 383.

[53] R. de Adelhart Toorop, F. Bazzocchi, L. Merlo and A. Paris, JHEP 1103 (2011) 035 Erratum: [JHEP 1301 (2013) 098] [arXiv:1012.1791].

[54] R. de Adelhart Toorop, F. Bazzocchi, L. Merlo and A. Paris, JHEP 1103 (2011) 040 [arXiv:1012.2091].

[55] I. de Medeiros Varzielas and D. Emmanuel-Costa, Phys. Rev. D 84 (2011) 117901 [arXiv:1106.5477].

[56] I. de Medeiros Varzielas, D. Emmanuel-Costa and P. Leser, Phys. Lett. B 716 (2012) 193 [arXiv:1204.3633].

[57] G. Bhattacharyya, I. de Medeiros Varzielas and P. Leser, Phys. Rev. Lett. 109 (2012) 241603 [arXiv:1210.0545].

[58] I. P. Ivanov and E. Vdovin, Eur. Phys. J. C 73 (2013) no.2, 2309 [arXiv:1210.6553].

[59] I. P. Ivanov and E. Vdovin, Phys. Rev. D 86 (2012) 095030 [arXiv:1206.7108].

[60] I. de Medeiros Varzielas and D. Pidt, J. Phys. G 41 (2014) 025004 [arXiv:1307.0711].

[61] I. de Medeiros Varzielas and D. Pidt, JHEP 1311 (2013) 206 [arXiv:1307.6545].

[62] V. Keus, S. F. King and S. Moretti, JHEP 1401 (2014) 052 [arXiv:1310.8253].

[63] V. Keus, S. F. King, S. Moretti and D. Sokolowska, JHEP 1411 (2014) 016 [arXiv:1407.7859].

[64] I. P. Ivanov and C. C. Nishi, JHEP 1501 (2015) 021 [arXiv:1410.6139].

[65] V. Keus, S. F. King, S. Moretti and D. Sokolowska, JHEP 1511 (2015) 003 [arXiv:1507.08433].

[66] M. Fallbacher and A. Trautner, Nucl. Phys. B 894 (2015) 136 [arXiv:1502.01829]. 
[67] I. P. Ivanov and J. P. Silva, arXiv:1512.09276.

[68] D. Emmanuel-Costa, O. M. Ogreid, P. Osland and M. N. Rebelo, JHEP 1602 (2016) 154 [arXiv:1601.04654].

[69] I. P. Ivanov and L. Lavoura, Eur. Phys. J. C 73 (2013) no.4, 2416 [arXiv:1302.3656].

[70] I. P. Ivanov and C. C. Nishi, Phys. Rev. D 82 (2010) 015014 [arXiv:1004.1799].

[71] I. P. Ivanov, JHEP 1007 (2010) 020 [arXiv:1004.1802].

[72] A. E. Carcamo Hernandez, I. de Medeiros Varzielas, S. G. Kovalenko, H. Ps and I. Schmidt, Phys. Rev. D 88 (2013) no.7, 076014 [arXiv:1307.6499].

[73] V. Keus, S. F. King and S. Moretti, Phys. Rev. D 90 (2014) no.7, 075015 [arXiv:1408.0796].

[74] C. C. Nishi, JHEP 1503 (2015) 034 [arXiv:1411.4909].

[75] I. de Medeiros Varzielas, O. Fischer and V. Maurer, JHEP 1508 (2015) 080 [arXiv:1504.03955].

[76] W. Grimus and M. N. Rebelo, Phys. Rept. 281 (1997) 239 [hep-ph/9506272].

[77] E. Ma and G. Rajasekaran, Phys. Rev. D 64 (2001) 113012 [hep-ph/0106291].

[78] C. Luhn, S. Nasri and P. Ramond, J. Math. Phys. 48 (2007) 073501 [hep-th/0701188].

[79] E. Ma, Phys. Lett. B 632 (2006) 352 [hep-ph/0508231].

[80] J. A. Escobar and C. Luhn, J. Math. Phys. 50 (2009) 013524 [arXiv:0809.0639].

[81] E. Ma, Mod. Phys. Lett. A 21 (2006) 1917 [hep-ph/0607056].

[82] C. C. Nishi, Phys. Rev. D 88 (2013) no.3, 033010 [arXiv:1306.0877].

[83] H. Ishimori, T. Kobayashi, H. Okada, Y. Shimizu and M. Tanimoto, JHEP 0904 (2009) 011 [arXiv:0811.4683]. 